\title{
Perioperative Blutungskomplikationen bei Netzhaut- und Glaukomeingriffen
}

\author{
INAUGURAL-DISSERTATION \\ zur Erlangung des Doktorgrades \\ der Medizinischen Fakultät der \\ Georg-August-Universität zu Göttingen
}

vorgelegt von

\author{
Anthea Klingelhöfer \\ aus Bremen
}

Göttingen 2021 


\section{Betreuungsausschuss}

Betreuer:

Ko-Betreuerin:

\section{Prüfungskommission}

Referent/in:

Ko-Referent/in:

Drittreferent/in:

Datum der mündlichen Prüfung:
Prof. Dr. Nicolas Feltgen

Prof. Dr. Dorothee Mielke

Prof. Dr. Nicolas Feltgen

Prof. Dr. Dorothee Mielke

Prof. Dr. Margarete Schön

14.07.2021 
Hiermit erkläre ich, die Dissertation mit dem Titel "Perioperative Blutungskomplikationen bei Netzhaut- und Glaukomeingriffen" eigenständig angefertigt und keine anderen als die von mir angegebenen Quellen und Hilfsmittel verwendet zu haben.

Göttingen, den

(Unterschrift) 
Die Daten, auf denen die vorliegende Arbeit basiert, wurden teilweise publiziert:

Lauermann P, Klingelhöfer A, Mielke D, van Oterendorp C, Hoerauf H, Striebe NA, Storch MW, Pfeiffer S, Koscielny J, Sucker C et al. (2021): Risk factors for severe bleeding complications in vitroretinal surgery and the role of antiplatelet or anticoagulant agents. Ophthalmol Retina (im Druck) 


\section{Inhaltsverzeichnis}

Abbildungsverzeichnis ..................................................................... III

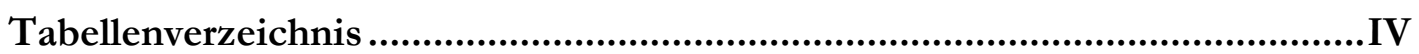

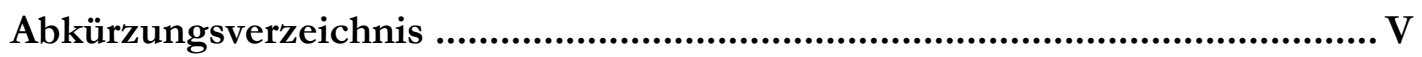

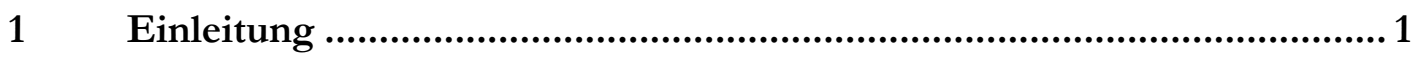

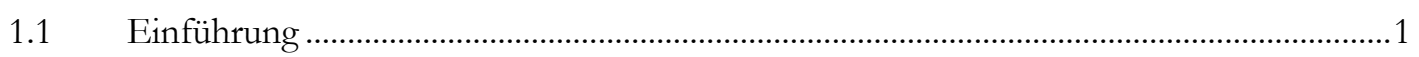

1.2 Perioperative Blutungskomplikationen bei chirurgischen Eingriffen ................................2

1.3 Perioperative Blutungskomplikationen in der Augenheilkunde..........................................

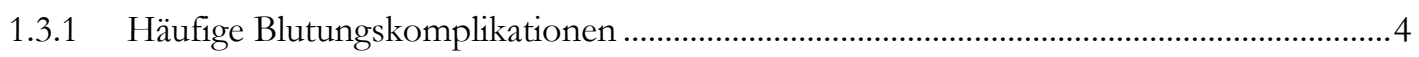

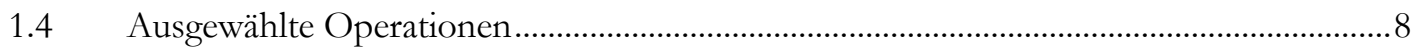

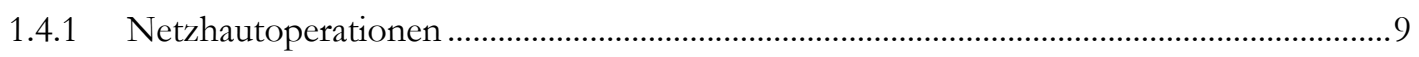

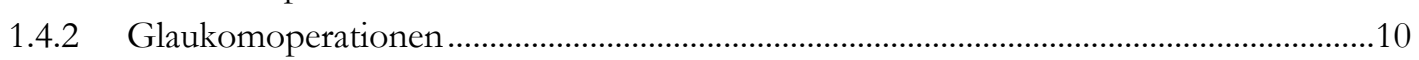

1.4.3 Anästhesieformen in der Ophthalmochirurgie ...............................................................12

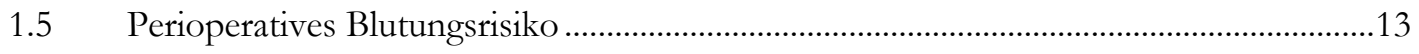

1.5.1 Präoperative Einschätzung des Blutungsrisikos .............................................................13

1.6 Ziele der vorliegenden Arbeit.........................................................................................18

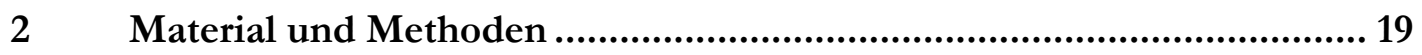

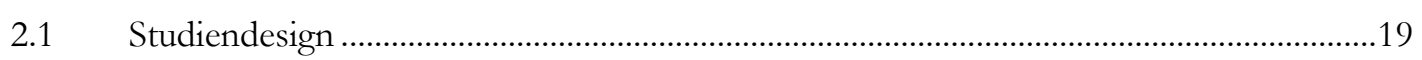

2.2 Ein- und Ausschlusskriterien.......................................................................................19

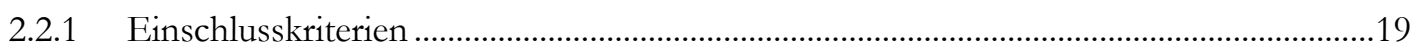

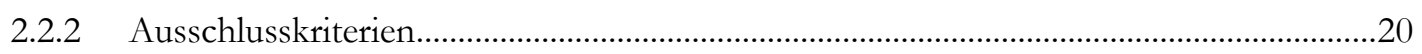

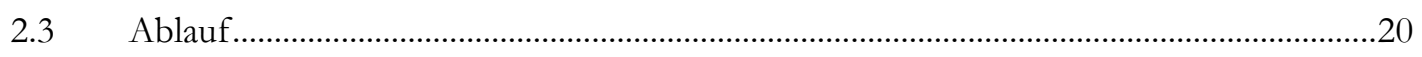

2.4 Verwendete Materialien und Definitionen ....................................................................21

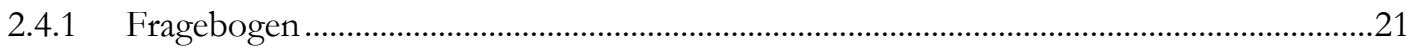

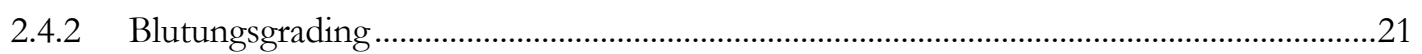

2.4.3 Definition relevanter Blutungskomplikationen ...........................................................23

2.5 Statistische Auswertung..........................................................................................2

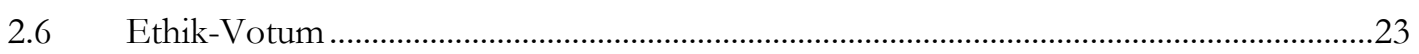

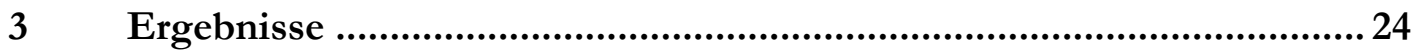

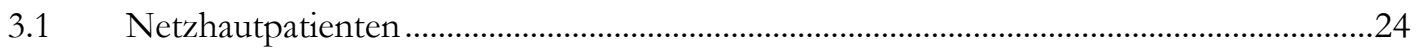

3.1.2 Relevante perioperative Blutungskomplikationen bei Netzhauteingriffen.......................30

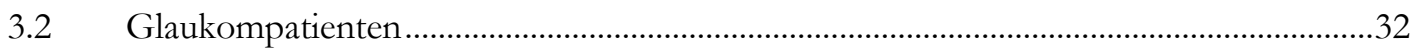

3.2.2 Relevante perioperative Blutungskomplikation bei Glaukomeingriffen...........................36

3.3 Mögliche Zusammenhänge und Risikofaktoren................................................................38

3.3.1 Mögliche übereinstimmende Risikofaktoren bei Netzhaut- und Glaukomoperationen

3.3.2 Mögliche Risikofaktoren bei Netzhautoperationen ........................................................4

3.3.3 Mögliche Risikofaktoren bei Glaukomoperationen..........................................................48 
3.4 Patienten mit Blutungskomplikationen...........................................................................50

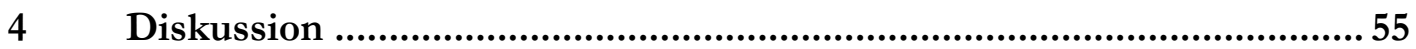

4.1 Anwendbarkeit des Fragebogens zur präoperativen Blutungsanamnese in der

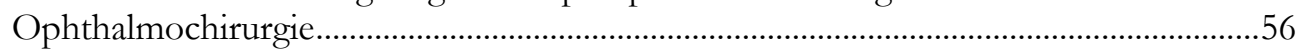

4.2 System zur Einteilung der Blutungsstärke von intraoperativen Blutungen.....................56

4.3 Risikofaktoren für ein erhöhtes Blutungsrisiko ...........................................................58

4.3.1 Risikofaktoren bei Netzhautoperationen.......................................................................58

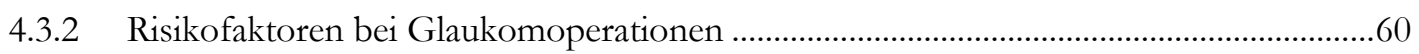

4.4 Erkenntnisse zu Blutungskomplikationen, Schussfolgerungen für den Umgang mit gerinnungsmodulierenden Medikamenten in der Augenchirurgie..................................61

$4.5 \quad$ Bewertung möglicher Fehlerquellen...............................................................................62

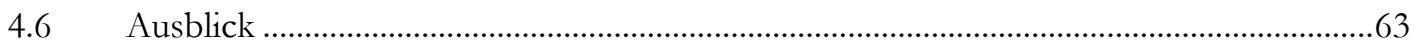

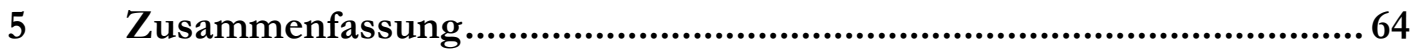

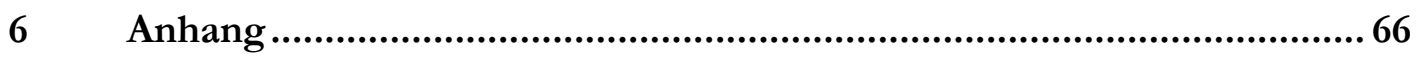

$7 \quad$ Literaturverzeichnis ................................................................ 71 


\section{Abbildungsverzeichnis}

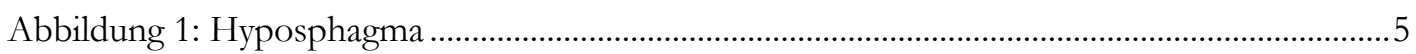

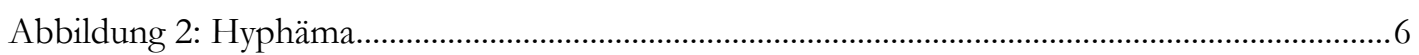

Abbildung 3: Funduskopiebild einer Glaskörperblutung.............................................................

Abbildung 4: Funduskopiebild einer subhyaloidalen Blutung bei Diabetischer Retinopathie......8

Abbildung 5: Klinisch validierter, standardisierter Blutungsanamnesebogen................................15

Abbildung 6: Begleiterkrankungen bei Patienten mit Netzhautoperationen .................................25

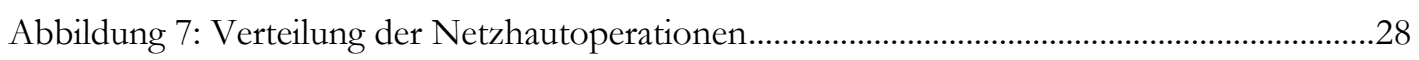

Abbildung 8: OP-Dauer bei Erst- und Wiederholungseingriffen bei Netzhautoperationen .....29

Abbildung 9: Nachblutungen bei Netzhautoperationen ..................................................................

Abbildung 10: Begleiterkrankungen bei Patienten mit Glaukomoperationen ................................33

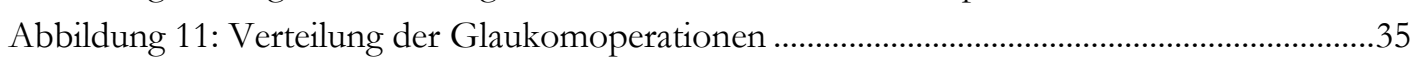

Abbildung 12: Nachblutungen bei Glaukomoperationen...............................................................

Abbildung 13: Blutungskomplikationen und Fragebogen-Score ....................................................40

Abbildung 14: Blutungskomplikationen und systolischer Blutdruck bei Glaukom- und Netzhautoperationen .............................................................................................4

Abbildung 15: Blutungskomplikationen und OP-Dauer bei Netzhaut- und

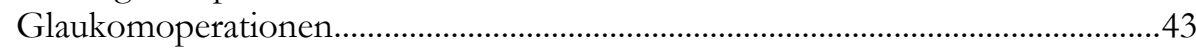

Abbildung 16: Blutungskomplikationen und Alter bei Netzhautoperationen ..............................44 


\section{Tabellenverzeichnis}

Tabelle 1: Zuordnung ophthalmochirurgischer Eingriffe zu Risikogruppen hinsichtlich des perioperativen Blutungsrisikos...

Tabelle 2: In die Studie eingeschlossene Netzhautoperationen ...................................................20

Tabelle 3: In die Studie eingeschlossene Glaukomoperationen ....................................................20

Tabelle 4: Verwendetes Blutungsgrading bei Netzhautoperationen ............................................22

Tabelle 5: Verwendetes Blutungsgrading bei Glaukomoperationen ..............................................22

Tabelle 6: Veränderung der Medikation bei Patienten mit einer Netzhautoperation...................26

Tabelle 7: Perioperative Blutdruckwerte der Netzhautpatienten ......................................................3

Tabelle 8: Intraoperative Blutungen bei Netzhautoperationen .........................................................

Tabelle 9: Veränderung der Medikation bei Patienten mit Glaukomoperationen ..........................34

Tabelle 10: Perioperative Blutdruckwerte der Glaukompatienten..................................................36

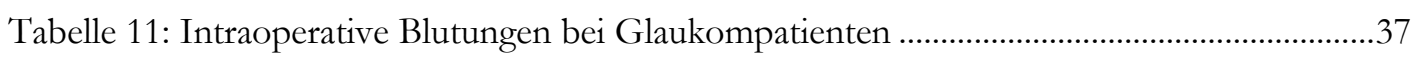

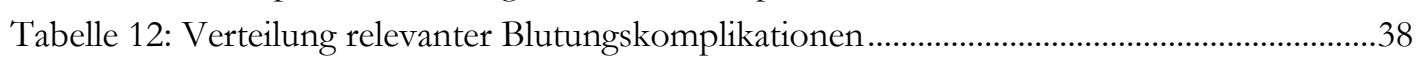

Tabelle 13: Blutungskomplikationen bei Netzhaut- und Glaukomoperationen............................38

Tabelle 14: Blutungskomplikationen und Anästhesieverfahren bei Netzhaut- und

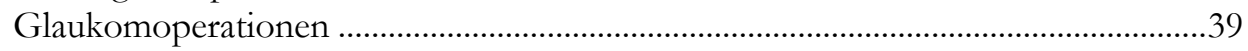

Tabelle 15: Patienten mit gerinnungsmodifizierender Dauermedikation ......................................41

Tabelle 16: Gerinnungsmodifizierende Medikamente und relevante Blutungskomplikationen bei Glaukom- und Netzhautoperationen ....................................................................42

Tabelle 17: Kardiovaskuläre Begleiterkrankungen bei Patienten mit Netzhautoperationen .......45

Tabelle 18: Blutungskomplikationen bei verschiedenen Netzhautoperationen ...........................47

Tabelle 19: Blutungskomplikationen und transsklerale Punktion bei eindellenden Netzhautoperationen...............................................................................................4

Tabelle 20: Blutungskomplikationen und Geschlecht bei Glaukomoperationen..........................48

Tabelle 21: Blutungskomplikationen und kardiovaskuläre Begleiterkrankungen bei Glaukomoperationen

Tabelle 22: Blutungskomplikationen bei verschiedenen Glaukomoperationen ............................50

Tabelle 23: Präoperative Merkmale der Patienten mit perioperativen Blutungskomplikationen

Tabelle 24: Intraoperative Merkmale von Patienten mit perioperativen

Blutungskomplikationen...........................................................................................52

Tabelle 25: Multivariate Analyse (logistische Regression) ...........................................................54 


\section{Abkürzungsverzeichnis}

$\begin{array}{ll}\text { ADP } & \text { Adenosindiphsopat } \\ \text { ASS } & \text { Acetylsalicylsäure } \\ \text { DOAK } & \text { Direkte orale Antikoagulantien } \\ \text { DOG } & \text { Deutsche Ophthalmologische Gesellschaft } \\ \text { INR } & \text { International Normalized Ratio } \\ \text { KHK } & \text { Koronare Herzkrankheit } \\ \text { pAVK } & \text { Periphere arterielle Verschlusskrankheit } \\ \text { ppV } & \text { Pars-plana-Vitrektomie } \\ \text { VKA } & \text { Vitamin-K-Antagonisten } \\ \text { TFH } & \text { Thrombozytenfunktionshemmer } \\ \text { TBVT } & \text { Tiefe Beinvenenthrombose }\end{array}$




\section{Einleitung}

\subsection{Einführung}

In Deutschland erhalten jährlich über eine Million Patienten ${ }^{1}$ einen ophthalmochirurgischen Eingriff. Durch den demographischen Wandel handelt es sich bei Patienten der Augenheilkunde zunehmend um ältere Menschen. Bereits mehr als die Hälfte der operierten Patienten in der Ophthalmologie war bereits 2010 älter als 70 (Wolfram und Pfeiffer 2012). Infolgedessen steigt auch der Anteil der Patienten mit relevanten kardiovaskulären Erkrankungen und demzufolge auch der Anteil derjenigen, die gerinnungsmodulierende Medikamente einnehmen.

Die Einnahme dieser Medikamente erhöht das Risiko für perioperative Blutungskomplikationen zum Beispiel bei neurochirurgischen Eingriffen oder großen Gefäßoperationen erheblich (Hoffmeister et al. 2010). Bei der Entscheidung, ob gerinnungshemmende Medikamente vor einem chirurgischen Eingriff abgesetzt werden müssen, muss die erhöhte Blutungsgefahr aber immer gegen das Risiko einer perioperativen thromboembolischen Komplikation abgewogen werden (Lock et al. 2018). Aus diesem Grund gibt es in vielen chirurgischen Fachgebieten besondere Empfehlungen für den Umgang mit gerinnungshemmenden Medikamenten (Eisele et al. 2014), in einigen Fachgebieten wurden auch Leitlinien formuliert (Douketis et al. 2012; Sporbeck et al. 2015). In der Augenheilkunde ist die Datenlage diesbezüglich unzureichend, evidenzbasierte Empfehlungen oder Leitlinien fehlen (Feltgen et al. 2018). In der Literatur gibt es Hinweise darauf, dass das Risiko für perioperative Blutungen sowohl bei Glaukom- (Law et al. 2008) als auch bei Netzhauteingriffen (Oh et al. 2011) durch die Einnahme von gerinnungsmodifizierenden Medikamenten steigt. Andererseits gibt es auch Untersuchungen, die keine relevante Zunahme von Blutungskomplikationen unter der Einnahme solcher Substanzen zeigen, durch die das Absetzen der Medikation gerechtfertigt wäre (Brown und Mahmoud 2011; Mason et al. 2011). Es gibt keine konsentierten Empfehlungen für die mögliche Umstellung der Gerinnungsmedikation (Feltgen et al. 2016).

1 Aus Gründen der Lesbarkeit wurde in der vorliegenden Arbeit die männliche Form personenbezogener Substantive und Pronomina verwendet. Dies impliziert jedoch keine Benachteiligung des weiblichen Geschlechts, sondern soll im Sinne der sprachlichen Vereinfachung als geschlechtsneutral zu verstehen sein. 
Somit werden Augenärzte in Deutschland häufig mit der Entscheidung allein gelassen, wie sie mit den Medikamenten umgehen sollen (Sucker et al. 2019). Eine Befragung unter Ophthalmochirurgen in England ergab, dass zwei Drittel aller Operateure bei Glaukomoperationen die Gerinnungsmedikation beibehalten (Bonhomme et al. 2013).

$\mathrm{Da}$ in der Augenchirurgie die in anderen Fachdisziplinen gültigen Kriterien für eine schwere Blutungskomplikation, wie etwa ein hämodynamisch relevanter oder lebensbedrohlicher Blutverlust, nicht eintreten können (Sucker et al. 2019), werden ophthalmochirurgische Operationen im Vergleich zu anderen Fachgebieten insgesamt als Eingriffe mit geringerem Blutungsrisiko zusammengefasst (Hoffmeister et al. 2010).

Um einen Überblick über die aktuelle Situation zu erhalten, führten Feltgen et al. 2018 unter Vertretern der DOG und ihrer assoziierten Gesellschaften eine Umfrage durch, in welcher unter anderem der Umgang der Ophthalmochirurgen mit gerinnungshemmender Medikation sowie deren Einschätzung des Blutungsrisikos bei bestimmten Eingriffen abgefragt wurden. In dieser Umfrage konnte ein Konsens erzielt werden, der die augenärztlichen Operationen in Eingriffe mit geringem oder hohem Blutungsrisiko einteilt. Bei einigen Eingriffen herrschte keine Einigkeit bezüglich der Einordnung des Risikos. Zusätzlich konnten in dieser Umfrage die Parameter ,Visusverlust ${ }^{\star}$ und ,Re-Operation aufgrund der Blutungskomplikation' als relevante Blutungskomplikationen für die Ophthalmochirugie definiert werden (Feltgen et al. 2018).

In der vorliegenden Studie liegt der Fokus gemäß der beschriebenen Einteilung auf Operationen, die der Umfrage zufolge mit einem höheren Risiko perioperativer Blutungskomplikationen assoziiert werden. Hierfür wurden Glaukom- und NetzhautEingriffe ausgewählt.

Zur Einschätzung des perioperativen Blutungsrisikos werden in anderen chirurgischen Fächern präoperativ standardisierte Fragebögen zur Blutungsanamnese eingesetzt, mit denen vorbestehende Gerinnungsstörungen aufgedeckt und daraus präventive Verhaltensmaßnahmen zur Minimierung des perioperativen Blutungsrisikos abgeleitet werden sollen (Pfanner et al. 2007). Da in der Augenheilkunde bisher noch kein derartiger Fragebogen für alle Patienten angewendet wird, sollte die Verwendung eines solchen in der vorliegenden Studie erprobt werden.

\subsection{Perioperative Blutungskomplikationen bei chirurgischen Eingriffen}

Betrachtet man die Gesamtheit aller in der Medizin durchgeführten chirurgischen Eingriffe, so treten intra- und perioperative Blutungen bei etwa 10-20\% auf. Ein Viertel dieser 
Blutungen kommt durch perioperative Gerinnungsstörungen zustande (Latza et al. 1999). Diese sind entweder durch angeborene oder erworbene Beeinträchtigungen der Hämostase oder durch die Einnahme von gerinnungshemmenden Medikamenten bedingt (Scherer und Giebler 2004). Durch perioperative Blutungskomplikationen steigen Mortalität und Morbidität der Patienten (Ghadimi et al. 2016) und der durch diese entstandene erhöhte Transfusionsbedarf führt zu einer hohen finanziellen Belastung des Gesundheitssystems und einer, wenn auch geringen, Infektionsgefahr (Latza et al. 1999).

\subsection{Perioperative Blutungskomplikationen in der Augenheilkunde}

Auch in der Augenheilkunde steigt die Bedeutung von Gerinnungsmodulatoren aufgrund des zunehmenden Patientenalters. Während jedoch der Umgang mit medikamentösinduzierten perioperativen Blutungskomplikationen in anderen operativen Fächern bereits etabliert ist, gibt es in der Augenheilkunde zu diesen Themen bislang kaum systematisch erhobene Daten. Dabei wäre auch bei ophthalmochirurgischen Eingriffen eine Stratifizierung hinsichtlich des jeweiligen Risikos von Blutungskomplikationen durchaus von klinischer Relevanz. Da Blutungskomplikationen in der Ophthalmochirurgie jedoch nur sehr selten vital bedrohlich werden (Sucker et al. 2019), muss der Schweregrad von Blutungskomplikationen nach anderen Kriterien beurteilt werden. Dies geschieht beispielsweise unter Berücksichtigung des Risikos einer persistierenden Verminderung der Sehkraft bis hin zum kompletten und irreversiblen Visusverlust oder etwa der Notwendigkeit einer Re-Operation infolge der Blutungskomplikation (Feltgen et al. 2018). In den meisten operativen Fachgebieten kann die klinische Relevanz über eine Quantifizierung des intraoperativen Blutverlustes beurteilt werden. Diese kann etwa durch das Ablesen der mithilfe des Saugers gesammelten Blutmenge, anhand der Anzahl der benötigten Bauchtücher oder durch intraoperative Hämoglobin-Messungen bestimmt werden (Piekarski et al. 2020). Da der Blutverlust in der Augenchirurgie nie hämodynamisch relevant ist, stellt die Quantifizierung der intraoperativ verlorenen Blutmenge hier kein geeignetes Maß für die klinische Relevanz der intraoperativen Blutung dar. Aus diesen Gründen soll im Rahmen dieser Arbeit ein Klassifikationssystem zur Einteilung von Blutungskomplikationen nach dem Schweregrad sowohl für Netzhaut- als auch für Glaukompatienten definiert und im klinischen Einsatz überprüft werden. Damit soll eine standardisierte und möglichst untersucherunabhängige Beurteilung von intraoperativen Blutungskomplikationen in der Ophthalmochirurgie erreicht werden.

In der Literatur werden zahlreiche Faktoren diskutiert, die ein erhöhtes Blutungsrisiko bei intraokularen Eingriffen zur Folge haben könnten. Dazu zählt unter anderem die gewählte 
Narkoseart. Es konnte etwa eine höhere Inzidenz perioperativer Blutungskomplikationen bei Augen mit Silikonölentfernung beobachtet werden, wenn der Eingriff in Intubationsnarkose (im Vergleich zur Lokalanästhesie) durchgeführt wurde (Stang und Bartz-Schmidt 2019). Darüber hinaus gilt auch ein hoher intraoperativer Blutdruck als möglicher Risikofaktor. In anderen Fachdisziplinen ist ein erhöhter intraoperativer Blutdruck ein wichtiger Risikofaktor für intraoperative Blutungen (Dünne et al. 2005), auch in der Augenheilkunde wird dies diskutiert (Agarwal et al. 2010). Im Rahmen dieser Arbeit haben wir diese und weitere Parameter präoperativ erhoben, um prospektiv deren Einfluss auf das perioperative Blutungsrisiko zu untersuchen.

\subsubsection{Häufige Blutungskomplikationen}

Im Rahmen der vorgestellten Eingriffe kann es zu Blutungskomplikationen kommen. Dabei ist zwischen verschiedenen Blutungsquellen zu unterscheiden.

\subsubsection{Bindehautblutungen}

Ein Hyposphagma ist eine in der Regel harmlose subkonjunktivale Blutung. Diese kann spontan auftreten, etwa bei älteren Menschen mit einer arteriellen Hypertonie. Ebenso kann ein Hyposphagma Folge einer Verletzung der Bindehaut durch eine Operation oder ein Trauma sein. Es wird in der Regel innerhalb von 1-2 Wochen resorbiert, weshalb keine spezifische Therapie nötig ist (Grehn 2012). 


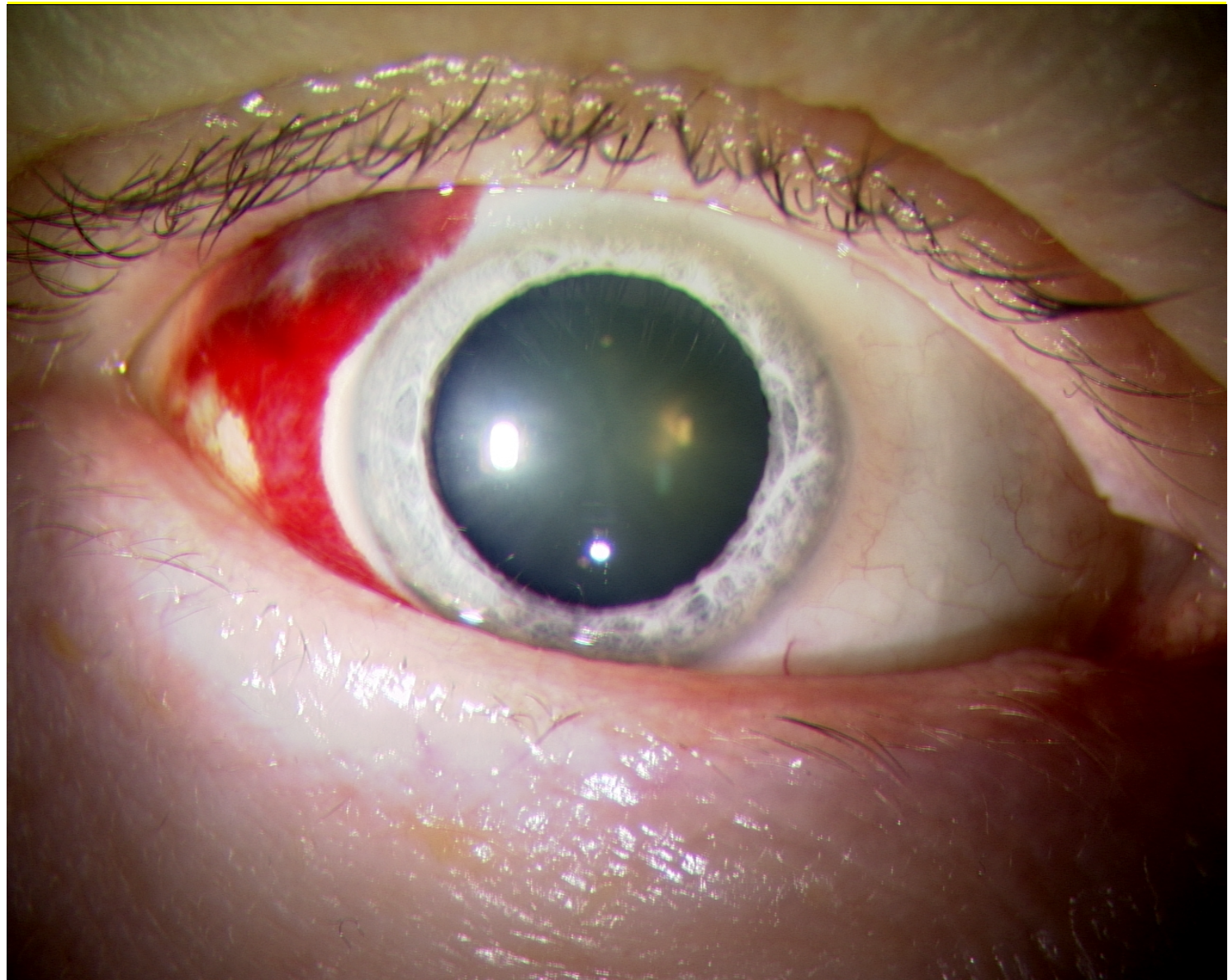

Abbildung 1: Hyposphagma Die Verwendung der erfolgt mit freundlicher Genehmigung von Prof. Dr. Nicolas Feltgen (UMG, Klinik für Augenheilkunde).

\subsubsection{Blutungen in der Vorderkammer}

Bei einem Hyphäma handelt es sich um eine Blutansammlung in der Vorderkammer. Es kann infolge eines chirurgischen Eingriffs oder eines Traumas auftreten, aber auch spontan, etwa im Rahmen von Gerinnungsstörungen oder der Einnahme von gerinnungshemmenden Substanzen. Die Blutungsquelle sind in der Regel Gefäße der Iris oder des Kammerwinkels. Eine ambulante Therapie ist häufig ausreichend, eine operative Therapie muss nur erwogen werden, wenn etwa der Augeninnendruck durch die Blutung stark erhöht ist und mit drucksenkenden Medikamenten nicht gesenkt werden kann (Walton et al. 2002). Zudem darf das Hornhautendothel nicht zu lange mit der Blutung in Kontakt stehen, da sich sonst Erythrozyten in das Endothel einbetten (sog. Hämatocornea). 


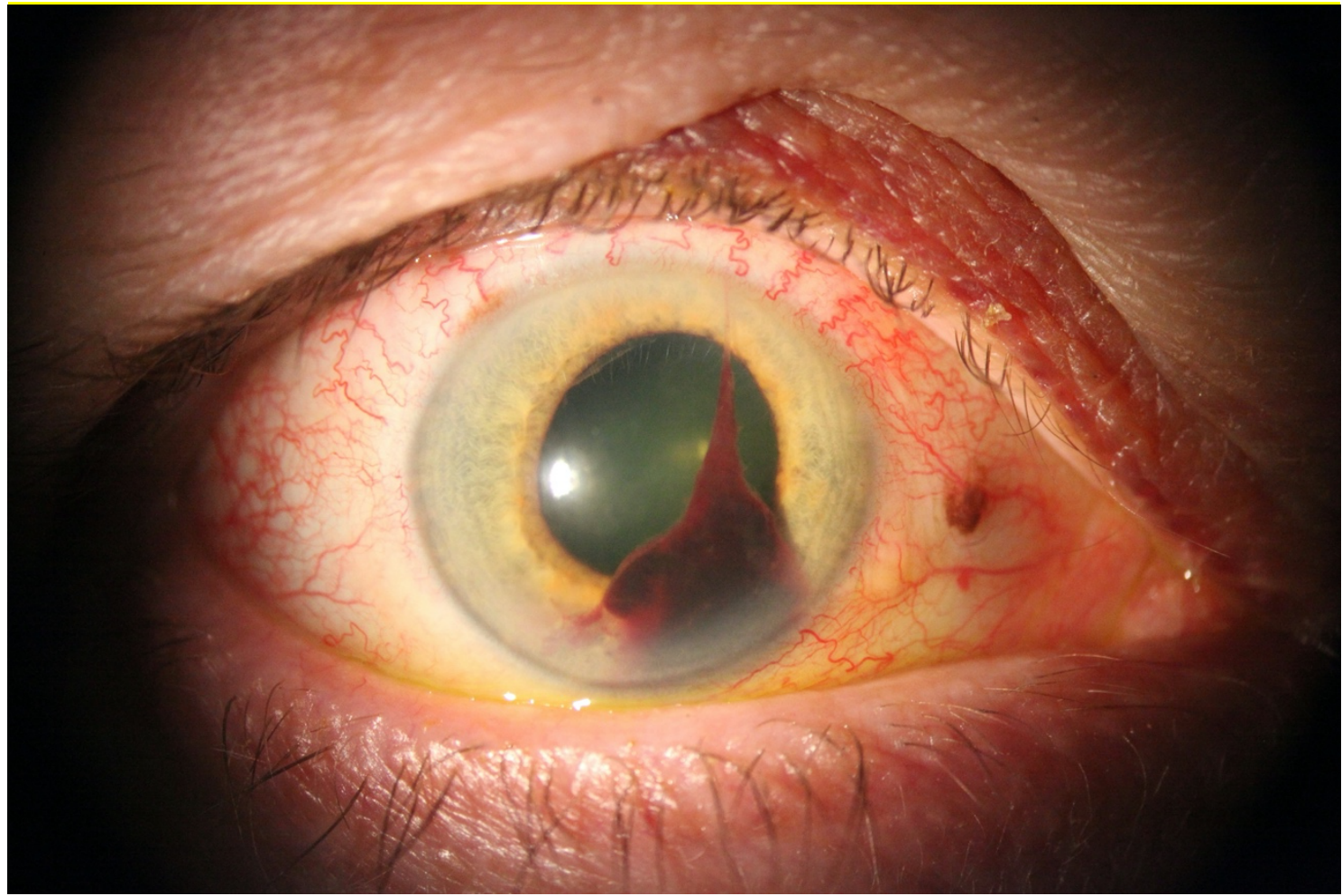

Abbildung 2: Hyphäma Die Verwendung der erfolgt mit freundlicher Genehmigung von Prof. Dr. Nicolas Feltgen (UMG, Klinik für Augenheilkunde).

\subsubsection{Blutungen im hinteren Augenabschnitt}

Blutungen im hinteren Augenabschnitt können den Glaskörperraum füllen (sog. Glaskörperblutung), zwischen Netzhaut und Glaskörper auftreten (sog. subhyaloidale Blutungen) oder aus der Aderhaut kommend unter der Netzhaut liegen (sog. subretinale oder Aderhautblutungen).

Glaskörperblutungen können beispielsweise nach Traumata, bei proliferativen Netzhauterkrankungen, Netzhautlöchern, oder in Zusammenhang mit subarachnoidalen Blutungen auftreten. Letzteres wird Terson-Syndrom genannt. Sie können sich beim Patienten als plötzlicher Visusverlust oder in Form eines sog. Rußregens äußern. Da eine Resorption möglich ist, wird häufig zunächst abgewartet. Bei fehlender Rückbildungstendenz oder besonders großen Blutungen kann eine Vitrektomie vorgenommen werden (Grehn 2019). Ein Funduskopiebild einer Glaskörperblutung ist in Abbildung 3 dargestellt.

Wie zuvor beschrieben kommt es bei einer subhyaloidalen Blutung zu einer Ansammlung von Blut zwischen Glaskörper und Netzhaut (Abbildung 4). Sie können bei Gefäßproliferationen, etwa bei Diabetischer Retinopathie oder als Folge von Venenastverschlüssen vorkommen. Ebenso können sie auch nach Valsalva-Manövern mit erhöhtem intrathorakalem oder intraabdominellem Druck auftreten, was dann als M. Purtscher bezeichnet. Meist führt dies zu einem unmittelbaren Sehverlust mit zentralen 
Gesichtsfeldausfall. Als Therapie stehen eine Laser-Membranotomie oder eine Pars-planaVitrektomie (ppV) zur Auswahl. Bei kleineren Blutungen kann, gerade bei jüngeren Patienten, auch eine Spontanresorption abgewartet werden (Völcker et al. 2012).

Subretinale Blutungen oder Aderhautblutungen können aus retinalen oder chorioidalen Gefäßen stammen. Sie treten häufig im Rahmen von Makuladegenerationen und anderen Erkrankungen mit Neovaskularisationen auf. Sie haben meist eine schlechte Visusprognose, können aber auch günstig verlaufen, was die Entscheidung über eine operative Therapie erschwert (Bopp 2012).

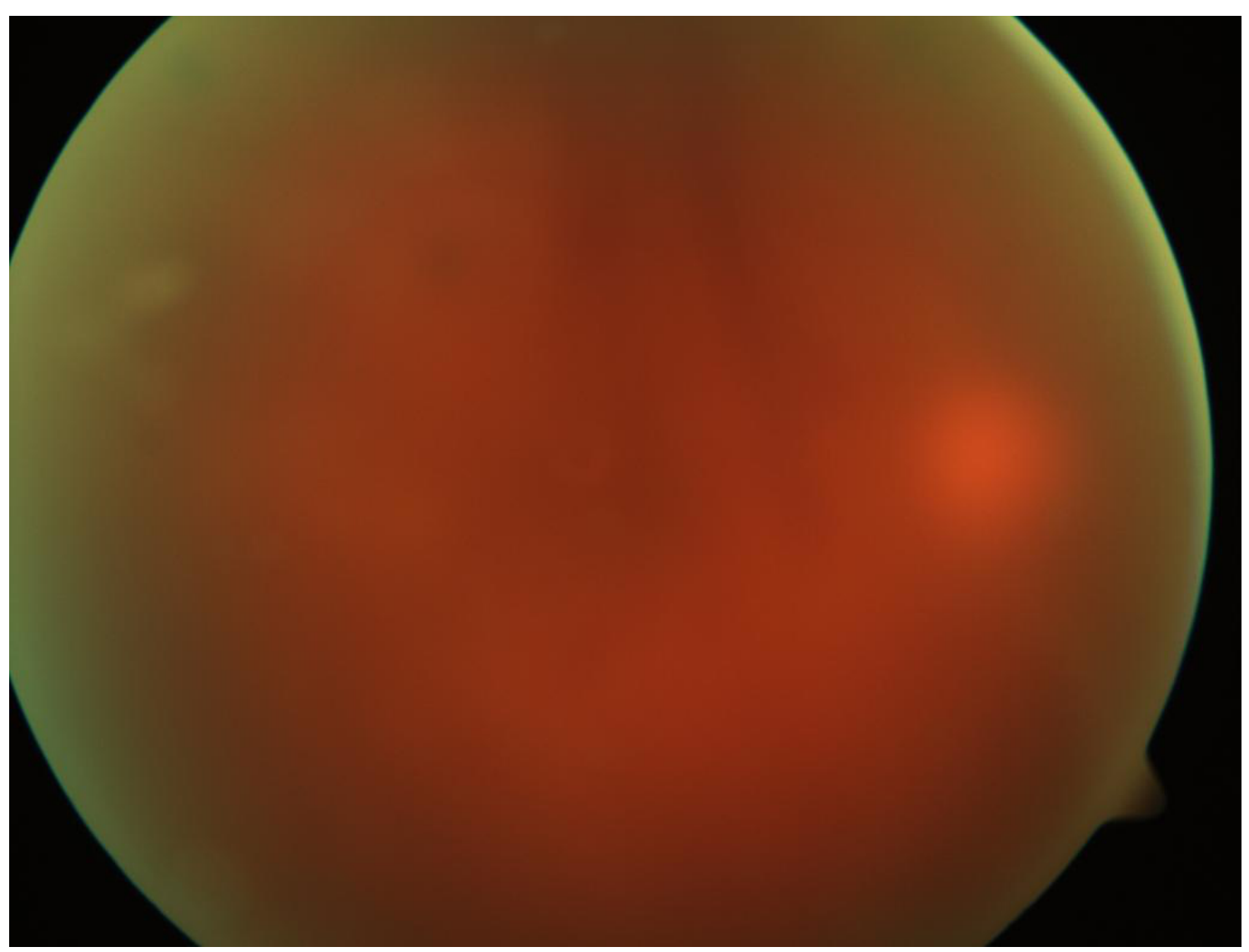

Abbildung 3: Funduskopiebild einer Glaskörperblutung Die Verwendung der erfolgt mit freundlicher Genehmigung von Prof. Dr. Nicolas Feltgen (UMG, Klinik für Augenheilkunde). 


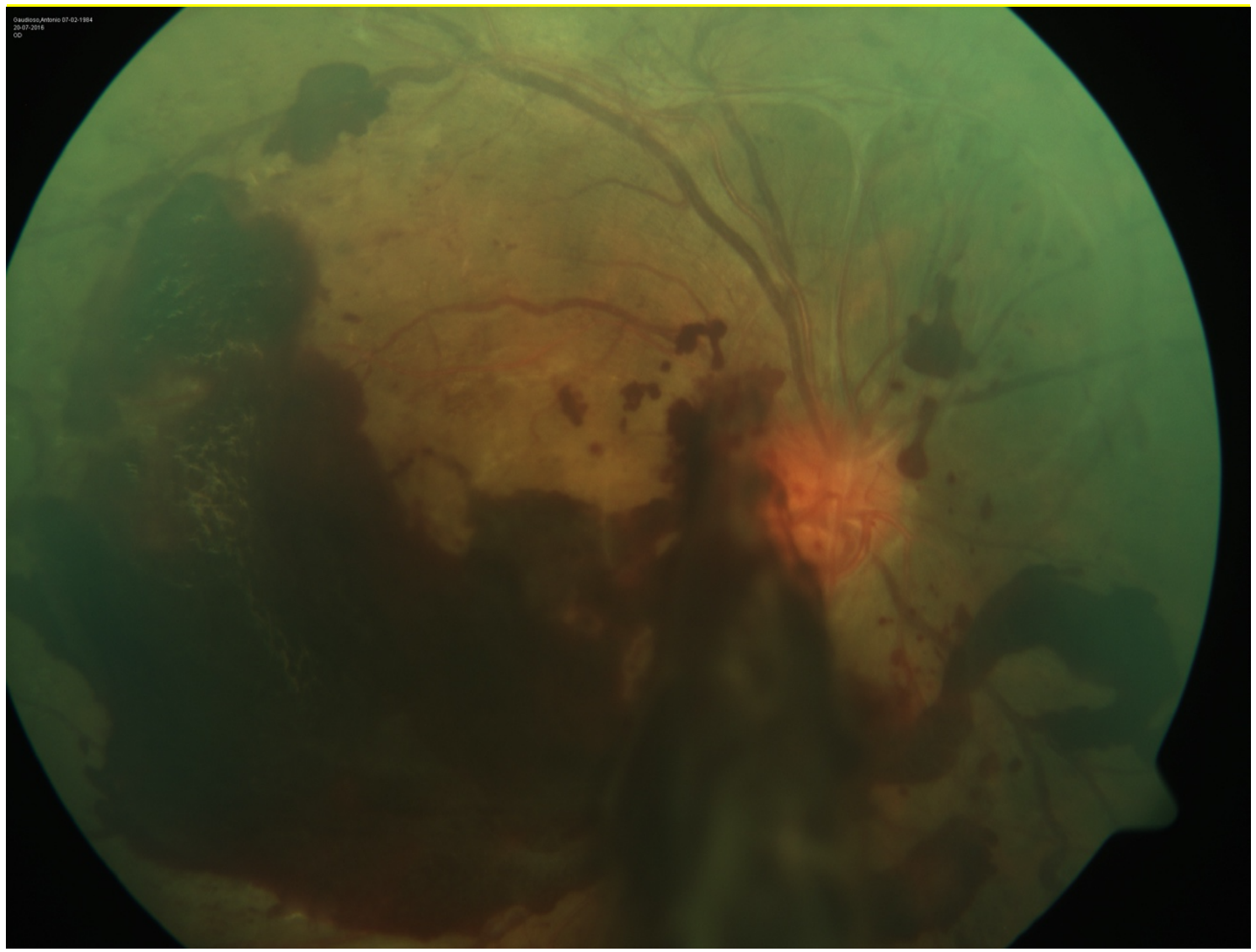

Abbildung 4: Funduskopiebild einer subhyaloidalen Blutung bei Diabetischer Retinopathie Die Verwendung der erfolgt mit freundlicher Genehmigung von Prof. Dr. Nicolas Feltgen (UMG, Klinik für Augenheilkunde).

\subsection{Ausgewählte Operationen}

Um eine möglichst hohe statistische Aussagekraft zu erreichen, entschieden wir uns bei der Auswahl der in dieser Studie zu berücksichtigenden Operationen für solche Eingriffe, die mit einem vergleichsweise hohen intraoperativen Blutungsrisiko einher gehen (Tabelle 1, rechte Spalte). 
Tabelle 1: Zuordnung ophthalmochirurgischer Eingriffe zu Risikogruppen hinsichtlich des perioperativen Blutungsrisikos

\begin{tabular}{|c|c|c|}
\hline $\begin{array}{l}\text { Eingriffe mit niedrigem } \\
\text { perioperativen } \\
\text { Blutungsrisiko }\end{array}$ & $\begin{array}{l}\text { Eingriffe mit intermediärem } \\
\text { perioperativen Blutungsrisiko }\end{array}$ & $\begin{array}{l}\text { Eingriffe mit hohem } \\
\text { perioperativen Blutungsrisiko }\end{array}$ \\
\hline $\begin{array}{ll}\text { - } & \text { Intravitreale operative } \\
\text { Medikamenteneingabe } \\
\text { (IVOM) } \\
\text { - } \quad \text { Netzhautkryokoagulation } \\
\text { - } \quad \text { Ketzhautlaserkoagulation } \\
\text { (sklerokorneal oder clear } \\
\text { cornea) } \\
\text { drucksenkende } \\
\text { Laserbehandlung } \\
\text { kleine lidchirurgische } \\
\text { Eingriffe wie } \\
\text { Wimpernepilation oder } \\
\text { Scherenschlag } \\
\text { einfachen } \\
\text { Muskeloperationen } \\
\text { perforierende } \\
\text { Keratoplastik (PKP) } \\
\text { phototherapeutischen } \\
\text { Keratektomie (PTK) } \\
\text { Descemetmembran } \\
\text { endothelialen } \\
\text { Keratoplastik (DMEK) }\end{array}$ & $\begin{array}{ll}\text { - } & \text { Retrobulbäranästhesie } \\
\text { - } & \text { Vitrektomie, Sonderformen } \\
\text { der Kataraktoperation (mit } \\
\text { Synechien) } \\
\text { - } & \text { Sonderformen der } \\
& \text { Glaukomoperation } \\
& \text { (Kanaloplastik, iStent) }\end{array}$ & $\begin{array}{l}\text { - Kammerwinkelchirurgische } \\
\text { Eingriffe (Trabektom- } \\
\text { Operation, Trabekulotomie) } \\
\text { - Goniotrepanation } \\
\text { - } \text { Glaukomchirurgische } \\
\text { Drainageimplantate } \\
\text { eindellende } \\
\text { Netzhautoperationen } \\
\text { - Vitrektomie bei neovaskulären } \\
\text { - } \quad \text { intraokulare Tumorchirurgie } \\
\text { - } \quad \text { ausgedehnte Lidoperationen } \\
\text { oder okuloplastische Eingriffe } \\
\text { Operationen der Orbita und } \\
\text { Muskeltransposition }\end{array}$ \\
\hline
\end{tabular}

Konsensentscheidung einer Umfrage nach (Feltgen et al. 2018). Die für die vorliegende Arbeit relevanten Eingriffe sind rot markiert.

Aus diesen Operationen haben wir die Gruppe der intraokularen Eingriffe, genauer der Netzhaut- und Glaukomoperationen, ausgewählt. Die orbitachirugischen Eingriffe und Lidoperationen, bei denen ebenfalls mit einer erhöhten Blutungsneigung gerechnet wird, werden in einer weiteren Promotionsarbeit behandelt. Im Folgenden werden nun die Krankheitsbilder und die zugehörigen Operationsverfahren vorgestellt.

\subsubsection{Netzhautoperationen}

$\mathrm{Zu}$ den in die Studie eingeschlossenen Netzhauterkrankungen gehören altersbedingte Veränderungen, wie eine Netzhautablösung, eine Makuladegeneration, ein Makulaforamen oder eine epiretinale Gliose. Außerdem wurden Patienten mit Gefäßerkrankungen der Netzhaut und Patienten mit einer diabetischen Retinopathie in die Studie aufgenommen. Als weitere Entität wurde entzündliche Veränderungen, wie die seltene Endophthalmitis, in die Studie einbezogen, wenn hierbei eine Vitrektomie erforderlich wurde

Für die meisten Indikationen ist eine sogenannte Pars-plana-Vitrektomie erforderlich. Netzhautablösungen können zudem noch mit eindellenden Operationsverfahren (Plombe 
oder Cerclage) versorgt werden. Manchmal ist eine Kombination beider Verfahren erforderlich.

Im Zuge einer Pars-plana-Vitrektomie wird der Glaskörper entfernt, um an die Netzhaut zu gelangen oder den Zug, den der Glaskörper auf die Netzhaut ausübt, zu verringern. Dazu werden nach der Eröffnung der Bindehaut durch die Sklera drei Zugänge mit einem Durchmesser von $0,9 \mathrm{~mm}$ bis $0,5 \mathrm{~mm}$ ins Innere des Augapfels geschaffen. Während mit einem Gerät der Glaskörper zerteilt und eingesaugt wird, wird mittels einer Infusion über einen anderen Zugang der Druck im Inneren aufrechterhalten. Durch ein Mikroskop kann der Operateur nun die Netzhaut und das Innere des Augapfels und die Netzhaut einsehen. Durch die verschiedenen Zugänge können dann, je nach Krankheitsbild, verschiedene Instrumente eingeführt, werden, um die jeweilige Pathologie zu behandeln. Zum Ende des Eingriffs wird der Bulbus entweder mit einem Luft-/Gasgemisch oder mit Silikonöl aufgefüllt, damit der Druck im Inneren des Auges kontinuierlich aufrechterhalten wird (Grehn 2019). Der Vorteil bei der Verwendung von kleineren Mikrokanülen (0,5 - 0,6 mm) ist, dass die dafür nötigen Inzisionen in der Sklera nach dem Eingriff nicht zugenäht werden müssen, sondern sich aufgrund der lamellären Stichtechnik alleine verschließen und vernarben (Eckardt 2005).

Die zweite Operationstechnik, welche ebenfalls bei Netzhautablösung zum Einsatz kommt, ist eine sklera-eindellende Operation. Bei diesem auch als Buckelchirurgie bezeichneten Operationsverfahren wird der Bulbus von außen komprimiert, wodurch der Zug des Glaskörpers an der Netzhaut abnimmt und diese sich wieder anlegen kann (Bopp 2019). Diese externe Kompression wird dann auch nach der Operation dauerhaft durch eine Plombe oder eine Cerclage aufrechterhalten, die auf den Bulbus aufgenäht oder wie ein Band um den Bulbus gelegt wird. Im Rahmen dessen muss manchmal transskleral subretinale Flüssigkeit entfernt werden, hierzu wird eine Stichinzision durchgeführt und als Punktion bezeichnet (Faude et al. 2002). Eindellende Operationsverfahren werden häufig bei eher jungen Patienten angewendet, die noch keine künstlichen Linsen besitzen. Vor allem bei diesen Patienten konnte eine Überlegenheit buckelchirurgischer Verfahren gegenüber der Vitrektomie bezüglich einer Linsentrübung bei gleichem anatomischen Operationserfolg gezeigt werden (Heimann et al. 2007).

\subsubsection{Glaukomoperationen}

Das Glaukom ist weltweit eine der häufigsten Ursachen für Erblindung und war Ende der 90er Jahre für 22\% der Erblindungen in Deutschland verantwortlich (Rohrschneider und Greim 2004). Es handelt sich dabei um eine heterogene Gruppe von Erkrankungen, die meist 
mit einem erhöhten Augeninnendruck einhergehen und zu Gesichtsfeldausfällen bis hin zur Erblindung führen können. Beim in die Studie eingeschlossenen Offenwinkelglaukom kommt es zu einer chronisch-progredienten schmerzlosen Sehnervenschädigung. Pathomorphologisch ist beim primären oder sekundären Offenwinkelglaukom eine strukturelle Veränderung des Trabekelwerkes zu beobachten, welche zu einer Behinderung des Kammerwasserabflusses führt (Jordan 2012). Ziel der Behandlung des Glaukoms ist die Senkung des Augeninnendrucks. Wenn medikamentöse Maßnahmen, wie etwa zur Verminderung der Kammerwasserproduktion, nicht mehr ausreichen, sind chirurgische Eingriffe zur Verbesserung des Kammerwasserabflusses die nächste therapeutische Stufe (Plange 2017).

Einige für diese Studie ausgewählte Operationen beinhalten die chirurgische Erweiterung des Kammerwinkels wie die Trabekulektomie. Bei diesen Operationen, auch als fistulierende Eingriffe bezeichnet, soll eine Lücke in der Sklera mittels einer Verbindung zwischen Vorderkammer und subkonjuntivalem Gewebe den dauerhaften und kontinuierlichen Abfluss des Kammerwassers sicherstellen. Die Trabekulektomie zählt hierbei zu den am häufigsten durchgeführten Eingriffen beim primären Offenwinkelglaukom (Taruttis et al. 2018).

Eine weitere kammerwinkelchirurgische Operationsmethode ist die sogenannte Viskokanaloplastik. Sie beinhaltet eine Erweiterung des Kammerwinkels mittels eines Fadens. Hierbei wird der Schlemm-Kanal zunächst sondiert. Anschließend wird durch diesen ein nicht resorbierbarer Faden geführt und dort unter Spannung verknotet. Dadurch soll der Abfluss des Kammerwassers an diesem Faden entlang gewährleistet werden (Dietlein et al. 2009).

Eine weitere Möglichkeit zur Senkung des Augeninnendrucks im Rahmen einer Glaukomerkrankung ist die Trabekulotomie. Bei diesem Operationsverfahren wird das veränderte Trabekelmaschenwerk um den Schlemm-Kanal eröffnet, um die Abflussöffnung des Kammerwassers zu vergrößern (Jordan et al. 2010).

Bei einer Trabekulotomie mit dem Trabektom wird mithilfe einer Elektroablation der Schlemm-Kanal eröffnet. So kann das Kammerwasser über die Vorderkammer in die Gefäßsysteme der Sklera abfließen (Wecker und Jordan 2015).

Eine Trabekulotomie kann aber auch ohne Elektroablation nur mit einem speziellen Messer, dem sogenannten Kahook Dual Blade erfolgen. Es besitzt eine Doppelklinge, mit der eine Mikroinzision des Schlemm-Kanals erfolgt. Dadurch wird ein verbesserter Kammerwasserabfluss geschaffen. Verglichen mit dem Trabektom scheinen Ergebnisse bei 
der Drucksenkung und aufgetretene Komplikationen ähnlich, es sind aber weniger Langzeitdaten vorhanden (van Oterendorp und Bahlmann 2019).

Glaukom-Drainage-Implantate (Ahmed/Baerveldt) können ebenfalls zu einer Ableitung des Kammerwassers eingesetzt werden. Operationsindikation ist meist die unzureichende Einstellung des Augeninnendrucks nach klassischen fistulierenden Eingriffen oder Druckanstiege bei Augen mit entzündlicher Begleiterkrankung. Um den Verschluss des Kammerwasserabfluss durch Narbengewebe zu verhindern, wird ein Schlauchsystem implantiert. Ein intraokular implantierter Silikonschlauch leitet das Kammerwasser in ein episklerales Reservoir (Reinthal et al. 2010). Ein Ventil sorgt dafür, dass der Druck postoperativ nicht zu stark sinkt. Vorteil dieser Eingriffe sind bessere Dosierungs- und Reversibilitätsmöglichkeiten gegenüber den zyklodestruktiven Substanzen, welche üblicherweise bei fistulierenden Eingriffen eingesetzt werden (Hille et al. 2005).

\subsubsection{Anästhesieformen in der Ophthalmochirurgie}

In der Ophthalmochirurgie werden verschiedene Anästhesieverfahren eingesetzt. Aufgrund des zumeist erhöhten Patientenalters bei einer ophthalmochirurgischen Operation und damit einhergehender erhöhter Prävalenz kardiovaskulärer Erkrankungen haben viele Patienten ein erhöhtes Risiko bei Durchführung einer Allgemeinnarkosen. Darüber hinaus können intraoperative Manipulationen am Auge und einige in der Augenheilkunde eingesetzte Medikamente Auswirkungen auf das kardiovaskuläre System haben. Neben der Intubationsnarkose werden daher auch lokale Verfahren eingesetzt (Heuser und Decker 1988). Die Auswahl des Anästhesieverfahrens richtet sich dabei unter anderem nach dem kardiovaskulären Risikoprofil des Patienten, der Art und der Länge des Eingriffs, der Kooperation und dem Wunsch des Patienten.

Zu den lokalen Anästhesieverfahren gehören die Retrobulbär- bzw. Parabulbäranästhesie, sowie die oberflächliche Analgesie mit Augentropfen. Bei der Retrobulbäranästhesie wird über einen inferotemporalen Zugang ein Lokalanästhetikum durch die Haut und das Bindegewebe hinter das Auge in den Konus und die äußeren Augenmuskeln injiziert. Dabei wird über eine Beteiligung mehrerer Hirnnerven eine Betäubung des Bulbus, eine Akinesie des Augapfels erreicht und ein Ausschalten der sensorischen fasern des Sehnerven erreicht (Weindler et al. 2004).

Für eine Parabulbäranästhesie wird ein Lokalanästhetikum in das Fettgewebe um die äußeren Augenmuskeln gegeben, der Muskelkonus aber nicht gesucht. Dadurch wird der Bulbus betäubt und die Beweglichkeit eingeschränkt. 
Eine weitere Alternative bietet die topische Anästhesie des Auges. Hierbei wird über ein Gel oder über Tropfen ein Lokalanästhetikum auf das Auge appliziert. Dies stellt eine sehr komplikationsarme Art der Anästhesie dar, erfordert aber eine gute Kooperation des Patienten, da die Beweglichkeit des Auges nicht eingeschränkt wird (Rex 2001). Zudem kann die topische Anästhesie nur bei Eingriffen des vorderen Augenabschnitts angewendet werden.

\subsection{Perioperatives Blutungsrisiko}

\subsubsection{Präoperative Einschätzung des Blutungsrisikos}

Um das Blutungsrisiko vor einer Operation einschätzen und Blutungen vorbeugen zu können, ist es nötig, eine mögliche Gerinnungsstörung oder Blutungsneigung bereits präoperativ festzustellen. Durch den immer häufigeren Einsatz gerinnungsmodulierender Medikamente kann die intra- und postoperative Blutungsneigung deutlich erhöht werden (Latza et al. 1999). Hierbei muss der Operateur die erhöhte Wahrscheinlichkeit einer perioperativen Blutung gegen die thromboembolischen Risiken abwägen, die durch die Umstellung oder das vorübergehende Absetzen der Medikamente entstehen.

\subsubsection{Labormedizinische Gerinnungsparameter}

Für die Einschätzung des perioperativen Blutungsrisikos werden präoperativ häufig Laborwerte erhoben, die auf eine erhöhte Blutungsneigung hindeuten oder eine Einschätzung der aktuellen Funktion von primärer und sekundärer Hämostase ermöglichen. $\mathrm{Zu}$ den üblicherweise verwendeten Gerinnungsparameter gehören die Thrombozytenzahl, der Quick-Wert sowie die aktivierte partielle Thromboplastinzeit (aPTT) (Eika et al. 1978), (Eberl et al. 2005). Ergibt dieses Labor-Screening den Verdacht auf eine Gerinnungsstörung, muss eine ausführlichere labormedizinsche Gerinnungsdiagnostik durchgeführt werden (Albert et al. 2009).

\subsubsection{Präoperative Blutungsanamnese}

In zahlreichen Studien konnte die Überlegenheit der standardisierten präoperativen Blutungsanamnese gegenüber der routinemäßigen Bestimmung von Standardgerinnungsparametern belegt werden, um das perioperative Blutungsrisiko einschätzen zu können. Durch die Erhebung einer standardisierten präoperativen Blutungsanamnese sollen häufige Gerinnungsstörungen erkannt werden. Bei auffälliger Anamnese können dann mithilfe eines gezielten Einsatzes von Gerinnungslaborparametern 
auch Störungen der Hämostase erkannt werden, die nicht durch die Standardgerinnungsparameter erfasst werden könnten (Pfanner et al. 2007). Beispielsweise können besonders Auffälligkeiten wie das häufige Von-Willebrandt-Jürgens-Syndrom und Störungen der Thrombozytenfunktion erkannt werden. In der Neurochirurgie oder der Allgemeinchirurgie wird die präoperative Blutungsanamnese bereits empfohlen oder regelhaft durchgeführt (Seicean et al. 2012; Schwenk 2019).

Insgesamt sollen mit der präoperativen Blutungsanamnese perioperative Blutungskomplikationen verringert werden. Darüber hinaus können über die sparsamere Anforderung von Gerinnungslaborwerten nur bei positivem Befund in der Blutungsanamnese und den verringerten Verbrauch von Blut- und Plasmaprodukten auch Behandlungskosten eingespart werden (Pfanner et al. 2007). Allein durch Vermeidung nicht unbedingt erforderlicher Laboranforderungen könnten deutschlandweit schätzungsweise 14,2 Millionen Euro jährlich eingespart werden (Koscielny et al. 2004). Nach der perioperativen Blutungsanamese und der individuellen Nutzen-Risiko-Analyse sollte dann die perioperative Anpassung der gerinnungsrelevanten Medikamente interdisziplinär entschieden werden (Pfanner et al. 2007).

In der vorliegenden Arbeit wurde für die Erhebung der präoperativen Blutungsanamnese der Patienten ein Fragebogen der Gruppe um den Berliner Hämostaseologen PD Dr. med. Jürgen Koscielny verwendet. Dieser wurde zur Abschätzung des perioperativen Blutungsrisikos erstellt (Pfanner et al. 2007) und prospektiv an der weltweit größten Kohorte, etwa 6000 Patienten, klinisch validiert. ${ }^{2}$ An 5649 erwachsenen Patienten wurde der Vorhersagewert von zwölf spezifischen anamnestischen Fragen zur Erkennung einer laboranalytisch messbaren Hämostasestörung beschrieben. Die Fragen hätten in dieser Kombination die höchste Wahrscheinlichkeit, Auffälligkeiten präoperativ zu erkennen. Dieser Fragebogen ist in der Abbildung 5 gezeigt.

\footnotetext{
2 Diese Informationen verdanke ich Herrn PD Dr. Koscielny (Charité - Universitätsmedizin Berlin,
} Gerinnungsambulanz mit Hämophiliezentrum), der mir dies schriftlich erläuterte. 


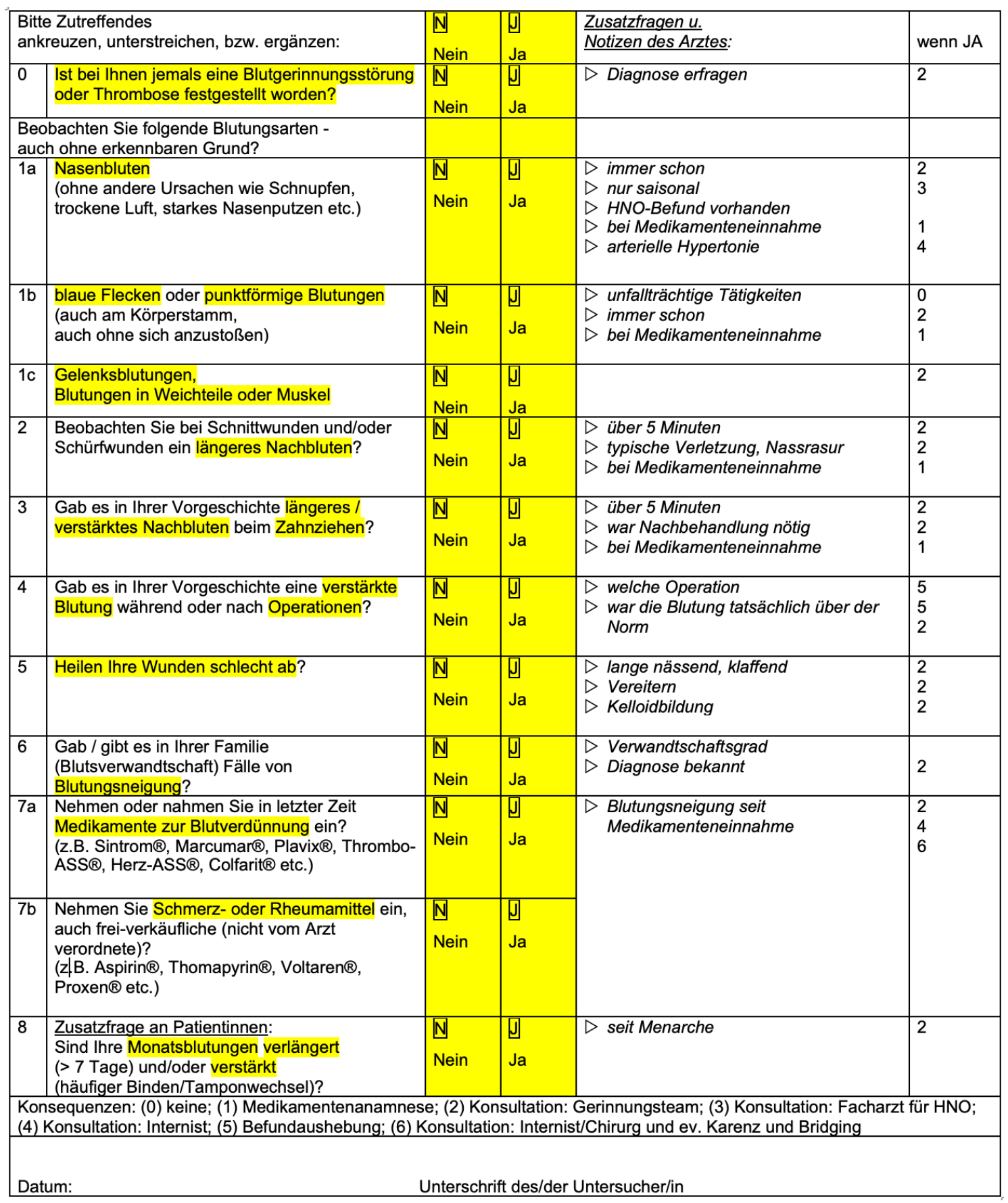

Abbildung 5: Klinisch validierter, standardisierter Blutungsanamnesebogen nach Pfanner et al. 2007; (gelb unterlegt: vom Patienten zu lesen und auszufüllen)

\subsubsection{Gerinnungsmodifizierende Medikamente}

Die Einnahme gerinnungsmodifizierender Medikamente wirkt sich auf das perioperative Blutungsrisiko aus, perioperatives Absetzen kann je nach Indikation das Risiko thromboembolischer Komplikationen erhöhen (Hoffmeister et al. 2010). Die Schwierigkeit im perioperativen Umgang mit diesen Substanzen liegt also darin, zwischen den Risiken bei Fortführung der Medikation beziehungsweise bei deren Absetzen abzuwägen. 
Bei den üblicherweise verwendeten Substanzen muss zwischen verschiedenen Medikamentengruppen unterschieden werden: Inhibitoren der Thrombozytenaggregation, sogenannte Thrombozytenfunktionshemmer (TFH), Direkte Orale Antikoagulantien (DOAK) und Vitamin-K-Antagonisten (VKA). Diese drei Gruppen werden im Folgenden kurz vorgestellt und die verschiedenen Empfehlungen zur Umstellung der Medikation erläutert. Ebenso erfragt wurde die Verwendung nicht-oraler applizierbarer Antikoagulantien wie Heparin. Darüber hinaus wurde anamnestisch die Einnahme einiger Nahrungsergänzungsmittel, wie Knoblauch und Gingko, erhoben, deren Einfluss auf die Blutgerinnung mittels verminderte Thrombozytenaktivierung belegt ist (Vogel Kahmann et al. 2011).

\subsubsection{Thrombozytenfunktionshemmer}

Bei den Thrombozytenfunktionshemmern ist zwischen Cyclooxygenasehemmern und P2Y12-Antagonisten zu unterscheiden.

Als typischer Vertreter der Cyclooxygenasehemmer wirkt Acetylsalicylsäure (ASS) über die irreversible Hemmung der Cyclooxygenase 1, welche dann zu einer verminderten Bildung von Thromboxan A2 führt. Dadurch wird die Aggregation als erster Schritt der Bildung von Thromben bei der primären Hämostase verhindert. Die Wirkung beginnt bereits nach zehn Minuten und hält über die etwa sieben bis zwölf Tage Lebensdauer der Thrombozyten an (Schlitt et al. 2013).

P2Y12-Hemmer wie Clopidogrel, Prasugrel oder Ticagrelor inhibieren über die irreversible Inaktivierung des thrombozytären P2Y12-Adenosindiphosphat(ADP)-Rezeptors die ADPvermittelte Thrombozytenaggregation und führen so ebenfalls zu einer Hemmung der primären Hämostase. Thrombozytenfunktionshemmer werden in der Primär- und Sekundärprophylaxe kardio- und zerebrovaskulärer Erkrankungen eingesetzt (Hein 2009). Bereits 2006 nahm ein Drittel der augenärztlichen Patienten im Vereinigten Königreich von Großbritannien Thrombozytenfunktionshemmer ein (Benzimra et al. 2009). Im Rahmen einer dualen Plättchenhemmung als Sekundärprophylaxe in den ersten Monaten nach einem Herzinfarkt oder einem Stenteinsatz kann auch eine Kombinationstherapie aus ASS und einem P2Y12-Hemmer wie Clopidogrel erfolgen (Lepper und Kelm 2007). Die Einnahme von Thrombozytenfunktionshemmern kann bei stabiler koronarer Herzkrankheit perioperativ ab einer Woche vor elektiven Eingriffen unterbrochen werden. Dies sollte jedoch nur nach ausführlicher Risiko-Nutzen-Analyse geschehen, da so perioperativ ein erhöhtes Risiko für kardiovaskuläre Ereignisse besteht. Zwar treten unter der Einnahme von 
Thrombozytenfunktionshemmern häufiger Blutungen, jedoch sehr selten schwere Blutungsereignisse auf (Hoffmeister et al. 2010).

\subsubsection{Vitamin-K-Antagonisten}

In Deutschland nimmt ca. 1\% der Bevölkerung VKA ein (Feltgen et al. 2016). VKA wie Phenprocoumon und Warfarin wirken über die Hemmung der hepatischen Produktion der Vitamin-K-abhängigen Gerinnungsfaktoren. Zur Kontrolle des Medikamentenspiegels und der Steuerung der Medikamenteneinnahme wird in der Regel der Wert der International Normalized Ratio (INR) verwendet. Hauptindikation ist die Sekundärprophylaxe akuter venöser Thromboembolien, zum Beispiel nach stattgehabter Thromboembolie, bei nicht einstellbarem Vorhofflimmern sowie einer mechanischen Herzklappe (Bauersachs 2003). Da VKA das perioperative Blutungsrisiko erheblich steigern, wird zur Normalisierung der Gerinnung bei größeren Operationen häufig bis zum Erreichen eines Ziel-INRs auf andere Gerinnungshemmer wie niedermolekulares oder unfraktioniertes Heparin umgestellt, dies wird als „Bridging“ bezeichnet (Hoffmeister et al. 2010). Bei Eingriffen mit geringem Blutungsrisiko, zu denen auch Katarakt- oder Glaukom-Operationen gezählt werden, ist allerdings aktuellen wissenschaftlichen Veröffentlichungen zufolge die Beibehaltung der Marcumar-Therapie dem Bridging überlegen (Giebl und Gürtler 2014).

\subsubsection{Direkte Orale Antikoagulantien}

DOAK wie Rivaroxaban, Apixaban, Edoxaban und Dabigatran sorgen über die Inhibition der Gerinnungsfaktoren Xa beziehungsweise IIa (bei Dabigatran) für eine Unterbrechung des intrinsischen und extrinsischen Weges der sekundären Hämostase (Merki et al. 2016). Sie werden ebenfalls zur Sekundärprophylaxe venöser Thromboembolien eingesetzt. DOAK haben eine größere therapeutische Breite als VKA und sind durch fehlende Notwendigkeit von Laborkontrollen für die verordneten Ärzte einfacher zu handhaben. Schwere und lebensbedrohliche Blutungskomplikationen treten bei beiden Substanzgruppen etwa gleich häufig auf (Connolly et al. 2009). Im Gegensatz zu den VKA gibt es für DOAK jedoch keine Standardlaborwerte zur Therapiesteuerung und sie können, im Gegensatz zu VKA, nicht antagonisiert werden. Sie sollen bei Operationen mit geringem Blutungsrisiko für ein Zeitfenster von 48 Stunden, 24 Stunden vor und 24 Stunden nach der Operation, abgesetzt werden (Sié et al. 2011). 


\subsection{Ziele der vorliegenden Arbeit}

Dieser prospektiven Arbeit liegen folgende Fragestellungen zugrunde:

1. Zunächst soll untersucht werden, ob der verwendete Fragebogen von Koscielny et al. zur präoperativen Blutungsanamnese auch in der Augenheilkunde sinnvoll eingesetzt werden kann.

2. Zusätzlich soll ein neu definiertes System zur Einteilung der Blutungsstärke erprobt werden, um relevante Blutungskomplikationen besser einteilen zu können.

3. Darüber hinaus soll überprüft werden, welche präoperativ erhobenen Merkmale mit einem erhöhten Blutungsrisiko einhergehen.

4. Insgesamt sollen durch die systematische Anlage einer spezifisch für die Augenheilkunde konzipierten Datengrundlage Erkenntnisse zu Blutungskomplikationen und dem Umgang mit gerinnungsmodulierenden Medikamenten in der Augenheilkunde gewonnen werden.

Die vorliegende Arbeit soll, wenn möglich, zur Ableitung von Empfehlungen für den klinischen ophthalmochirurgischen Alltag beitragen sowie Anhaltspunkte für weitere Forschungsansätze geben. 


\section{$2 \quad$ Material und Methoden}

\subsection{Studiendesign}

Die vorliegende prospektive Studie soll perioperative Blutungskomplikationen bei ophthalmochirurgischen Eingriffen, vor allem im Zusammenhang mit gerinnungsbeeinflussenden Medikamenten, erfassen. Die Studie mit insgesamt geplanten 1000 Patienten wurde in zwei Projekte mit je 500 Patienten unterteilt. Dabei wurden zwischen der extraokularen Augenchirurgie mit orbita- und lidchirurgischen Eingriffen sowie der intraokularen Augenchirurgie mit Glaukom- und Netzhauteingriffen unterschieden. In der vorliegenden Arbeit wurden ausschließlich intraokulare Eingriffe untersucht. Es handelt sich um die erste prospektive Untersuchung eines konsekutiven Patientenkollektivs auf diesem Themengebiet.

\subsection{Ein- und Ausschlusskriterien}

\subsubsection{Einschlusskriterien}

Die Kriterien zur Aufnahme in die Studie umfassten die Volljährigkeit und die schriftliche Einverständniserklärung der Patienten zur freiwilligen Teilnahme an der Studie. Darüber hinaus war die Indikation einer der ausgewählten Glaukom- oder Netzhautoperationen Voraussetzung für den Einschluss in die Studie. Eine Übersicht der Operationen, die zum Einschluss in die Studie berechtigten, ist in der Tabelle 2 und Tabelle 3 dargestellt. 
Tabelle 2: In die Studie eingeschlossene Netzhautoperationen

\begin{tabular}{|c|c|}
\hline Operationstyp & Operationen \\
\hline $\begin{array}{l}\text { Pars-Plana- } \\
\text { Vitrektomien }\end{array}$ & $\begin{array}{ll}\text { - } & \text { ppV bei Makulaforamen } \\
\text { - } & \text { ppV bei epiretinaler Gliose } \\
\text { - } & \text { ppV bei Netzhautablösung } \\
\text { - } & \text { ppV bei diabetischer Retinopathie } \\
\text { - } & \text { ppV bei submakulärer Blutung } \\
\text { - } & \text { ppV bei Glaskörpertrübung } \\
\text { - } & \text { ppV bei Entzündung } \\
\text { - } & \text { ppV bei Subluxation der Linse } \\
\text { - } & \text { Ölentfernung }\end{array}$ \\
\hline $\begin{array}{l}\text { eindellende } \\
\text { Operationen }\end{array}$ & $\begin{array}{ll}\text { - } & \text { Plomben- und/ oder Cerclageoperation bei Netzhautablösung } \\
\text { - } & \text { Kombinationseingriff (Eindellung und ppV) }\end{array}$ \\
\hline
\end{tabular}

Tabelle 3: In die Studie eingeschlossene Glaukomoperationen

\begin{tabular}{|l|cl|}
\hline Operationstyp & Operationen \\
\hline Fistulierende & $\bullet$ & Trabekulektomie \\
Operationen & & \\
\hline Minimalinvasive & $\bullet$ & Trabektom \\
Glaukomchirurgie & $\bullet$ & Kahook \\
& $\bullet$ & Viskokanaloplastik \\
& $\bullet$ & Ventil-Implantation (Ahmed, Baerveldt) \\
\hline
\end{tabular}

\subsubsection{Ausschlusskriterien}

Nicht in die Studie eingeschlossen werden konnten Patienten mit eingeschränkter Einwilligungsfähigkeit (z. B. Patienten mit gesetzlichem Betreuer) oder fehlender schriftlicher Einwilligung.

Nachträglich von der Teilnahme an der Studie ausgeschlossen wurden Patienten, deren Operation nicht durchgeführt wurde (z. B. verschobene Operation, geänderte Indikation), oder die ihre Einwilligungserklärung zurückzogen.

\subsection{Ablauf}

Die in die Studie eingeschlossenen Patienten wurde vor der Operation anhand des Fragebogens nach Koscielny et al. befragt. Neben dem Fragebogen zur präoperativen 
Blutungsanamnese wurden kardiovaskuläre Begleiterkrankungen sowie die Gerinnung beeinflussende Medikamente erfragt.

Unmittelbar nach dem Eingriff wurde durch den Operateur ein Arbeitsblatt ausgefüllt, auf welchem neben dem vorgestellten Blutungsgrading weitere Eckdaten der Operation wie deren Dauer und die durchgeführte Narkoseart erfasst wurden. Zudem wurden die intraoperativen und unmittelbar postoperativen Blutdruckwerte dokumentiert. Im Rahmen der Entlassung oder der Nachuntersuchung wurden darüber hinaus postoperative Komplikationen wie etwa Nachblutungen erfasst. Die verwendeten Arbeitsblätter sind im Anhang zu finden.

\subsection{Verwendete Materialien und Definitionen}

\subsubsection{Fragebogen}

In der vorliegenden Arbeit wurde der zuvor vorgestellte Fragebogen zur präoperativen Blutungsanamnese verwendet. Es sollte der prognostische Wert der bei der Befragung mittels des Fragebogens erreichten Punktzahl für das Auftreten relevanter perioperativer Blutungskomplikationen untersucht werden. Außerdem sollten die zwölf Fragen einzeln auf ihre jeweilige Bedeutung für die Abschätzung des Blutungsrisikos in der Augenheilkunde untersucht werden. Der Fragebogen ist in Abbildung $5 \mathrm{zu}$ finden. Der Fragebogen wurde im Rahmen der Erhebung der präoperativen Daten von mir während der Befragung der Patienten ausgefüllt.

\subsubsection{Blutungsgrading}

Für eine standardisierte Beurteilung der zu untersuchenden Blutungskomplikationen war eine einheitliche Klassifikation erforderlich. Dazu wurde, sowohl für die Glaukom- als auch für die Netzhautoperationen, in Zusammenarbeit mit den operierenden Augenärzten der Universitätsmedizin Göttingen für beide Operationsformen jeweils eine Ordinalskala von 0 bis 5 erarbeitet. Für die Netzhautoperationen wurde jeweils eine eigene Einteilung für Glaskörperoperationen und für buckelchirurgische Eingriffe (eindellende Operationsverfahren) erstellt. Dabei wurden im Konsens aller Operateure zunächst eine minimale und eine maximale Stufe festgelegt. Anschließend einigte man sich auf eine geeignete Differenzierung innerhalb dieser Grenzen. Gemäß dieser Einteilung nach der Blutungsstärke bedeutete die Stufe 0, dass es keine intraoperative Blutung gab und die Stufe 5, dass die Operation aufgrund der Blutungskomplikationen abgebrochen werden musste. Das erarbeitete Einstufungssystem ist in Tabelle 4 und Tabelle 5 aufgeführt. Zudem wurden 
die Blutungen nach der Quelle der Blutung differenziert: Kammerwinkel/Iris, Sklera/Ziliarkörper und Aderhaut. Zusätzlich wurden bei Netzhautoperationen auch noch die Netzhaut- und bei Glaukomeingriffen die Bindehaut als Blutungsquelle unterschieden. Bei buckelchirurgischen Operationen wurde zusätzlich erhoben, ob bei der Eindellung eine transsklerale Punktion erfolgt war.

Tabelle 4: Verwendetes Blutungsgrading bei Netzhautoperationen

\begin{tabular}{|c|c|c|}
\hline Blutungsstärke & Definition bei Vitrektomie & Definition bei eindellender Operation \\
\hline 0 & keine Blutung & keine Blutung \\
\hline 1 & $\begin{array}{l}\text { spontan sistierende Blutung ohne } \\
\text { Reduktion des Einblicks }\end{array}$ & $\begin{array}{l}\text { umschriebene/punktuelle } \\
\text { subretinale Blutung in der Peripherie }\end{array}$ \\
\hline 2 & $\begin{array}{l}\text { Blutung, die nach vorübergehender } \\
\text { Erhöhung des Irrigationsdrucks zum } \\
\text { Stillstand kommt, spontan sistierende } \\
\text { Blutung mit Reduktion des Einblicks }\end{array}$ & $\begin{array}{l}\text { flächige subretinale Blutung auf die } \\
\text { Peripherie begrenzt }\end{array}$ \\
\hline 3 & $\begin{array}{l}\text { Blutung, für die Endodiathermie } \\
\text { eingesetzt werden muss }\end{array}$ & $\begin{array}{l}\text { flächige subretinale Blutung bis an die } \\
\text { Gefäßbögen ohne Beteiligung des } \\
\text { hinteren Pols }\end{array}$ \\
\hline 4 & $\begin{array}{l}\text { Blutung trotz Endodiathermie, die nur } \\
\text { durch direkte Kompression gestillt } \\
\text { werden kann (z. B. Staubsauger auf } \\
\text { retinales Gefäß) }\end{array}$ & $\begin{array}{l}\text { flächige subretinale Blutung mit } \\
\text { Beteiligung des hinteren Pols }\end{array}$ \\
\hline 5 & nicht stillbare Blutung & subretinale Massenblutung \\
\hline
\end{tabular}

Tabelle 5: Verwendetes Blutungsgrading bei Glaukomoperationen

\begin{tabular}{|c|l|}
\hline Blutungsstärke & Definition \\
\hline $\mathbf{0}$ & $\begin{array}{l}\text { keine Blutung } \\
\text { spontan sistierende Blutungen }\end{array}$ \\
\hline $\mathbf{1}$ & $\begin{array}{l}\text { Blutungen, die den Operationsverlauf stören, die aber spontan bzw. ohne Einsatz von } \\
\text { Diathermie sistieren (inkl. Applikation von Adrenalin, vorübergehende Erhöhung des } \\
\text { Infusionsdrucks) }\end{array}$ \\
\hline $\mathbf{3}$ & $\begin{array}{l}\text { Blutung, für die Diathermie eingesetzt werden muss, um danach Operation störungsfrei } \\
\text { fortzusetzen }\end{array}$ \\
\hline $\mathbf{4}$ & $\begin{array}{l}\text { Blutung, die trotz Diathermie die Operation so stark behindert, dass diese nicht mehr } \\
\text { weitergeführt werden kann }\end{array}$ \\
\hline $\mathbf{5}$ & \begin{tabular}{l} 
massive, nicht stillbare Blutung inklusive Aderhautblutung \\
\hline
\end{tabular}
\end{tabular}




\subsubsection{Definition relevanter Blutungskomplikationen}

Für eine Auswertung der Blutungskomplikationen war es erforderlich, zu definieren, ab welcher Stärke eine Blutungskomplikation als relevant bewertet werden sollte. Hierzu übernahmen wir die bei der bereits zitierten Umfrage von Feltgen et al. 2018 erarbeitete konsentierte Definition, derzufolge eine dauerhafte Visusminderung und/oder die aus einer Blutung resultierende Notwendigkeit einer Re-Operation als relevante Blutungskomplikation definiert war (Feltgen et al. 2018). Zudem galt es auch in Bezug auf das Blutungsgrading relevante intraoperative Blutungen abzugrenzen. Als Kriterium hierfür wurde in Abstimmung mit den operierenden Augenchirurgen die Stufe 3 und höher festgelegt (siehe obenstehende Tabelle 4, Tabelle 5).

\subsection{Statistische Auswertung}

Die statistische Auswertung erfolgte in Kooperation mit Herrn Sebastian Pfeiffer aus dem Institut für Medizinische Statistik der Georg-August-Universität Göttingen. Für die statistische Auswertung wurde die Software SAS, Version 9.4 (2013), genutzt. Es wurden der T-Test, der Chi-Quadrat-Test und der exakte Test nach Fischer verwendet. Die Daten wurden mittels absoluter und relativer Häufigkeiten, Mittelwert und Standardabweichung sowie Median und Quartilen dargestellt.

\subsection{Ethik-Votum}

Die geplante Studie wurde der Ethik-Kommission der Universitätsmedizin Göttingen im Frühjahr 2018 vorgelegt und im April 2018 unter der Antragsnummer 15/6/18 genehmigt. 


\section{Ergebnisse}

In die vorliegende Studie wurden zwischen Februar 2019 und Dezember 2020 insgesamt 501 Patienten konsekutiv eingeschlossen. Die Eingriffe an den Studienteilnehmern wurden von verschiedenen auf Netzhaut- und Glaukomchirurgie spezialisierten Operateuren der Universitätsmedizin Göttingen durchgeführt. Nach Ausschluss der Patienten, bei denen kein Eingriff erfolgen musste oder ihre Einwilligung zurückzogen, gingen schließlich die Daten von insgesamt 463 Patienten in die Auswertung ein. Davon waren 374 (80,8\%) Patienten mit einer Netzhautoperation, $89(19,2 \%)$ Glaukompatienten. Im Folgenden werden die Ergebnisse für beide Patientengruppen zunächst separat vorgestellt und ausgewertet.

\subsection{Netzhautpatienten}

Von den 374 Patienten an der Netzhaut operierten Patienten waren 184 (49,2\%) weiblich, $190(50,8 \%)$ männlich. Es wurde in 185 Fällen (49,5\%) das rechte Auge und in 189 Fällen $(50,5 \%)$ das linke Auge operiert. Das Patientenalter zum Operationszeitpunkt variierte von 24 bis 94 Jahren (Mittelwert 67,6 Jahre, Standardabweichung 12,9).

\subsubsection{Kardiovaskuläre Begleiterkrankungen}

Kardiovaskuläre Begleiterkrankungen hatten 262 (70,1\%) der Patienten. Die häufigste Erkrankung war dabei die arterielle Hypertonie, die bei 228 (61,0\%) der Patienten diagnostiziert wurde. Danach folgten Patienten mit Diabetes mellitus (75 Patienten/20,1\%) oder einer koronaren Herzkrankheit (KHK) (41/11,0\%). Bei vielen Patienten lagen mehrere der erhobenen kardiovaskulären Begleiterkrankungen vor. 112 (30\%) Studienteilnehmer hatten keine diagnostizierte Erkrankung. Eine Übersicht der häufigsten Begleiterkrankungen ist in der untenstehenden Abbildung 6, eine Auflistung aller erhobenen Begleiterkrankungen in der Tabelle 17 auf Seite 45 dargestellt. 


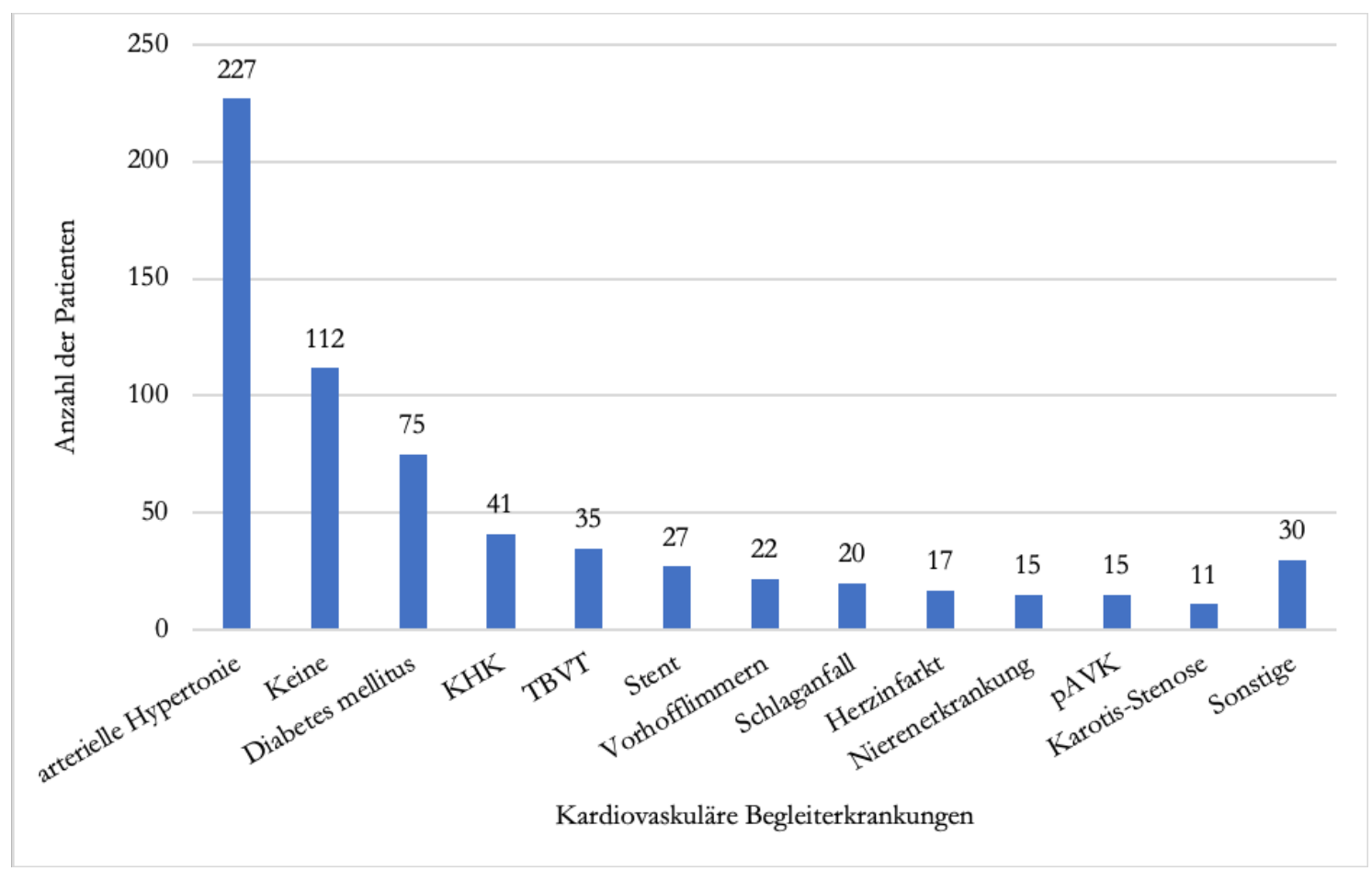

Abbildung 6: Begleiterkrankungen bei Patienten mit Netzhautoperationen ( $\mathrm{n}=617$,

Mehrfachnennungen möglich)

\subsubsection{Einnahme gerinnungsmodifizierender Medikamente}

Ein Anteil von 134 (35,8\%) der Patienten waren zum Operationszeitpunkt mit gerinnungsmodifizierenden Medikamenten vorbehandelt. Der TFH ASS wurde am häufigsten eingesetzt (89 Patienten/66,4\%). 15 (11,2\%) Probanden nahmen das NOAK Apixaban und 14 (10,4\%) den VKA Phenprocoumon ein. 240 (64,2\%) Studienteilnehmer wurden nicht mit gerinnungshemmenden Substanzen behandelt. Bei 82 Patienten mit gerinnungshemmender Medikation wurden die Substanzen nicht abgesetzt. Bei 47 Patienten wurde die Medikation abgesetzt und bei sieben Patienten wurde ein Bridging mit Heparin durchgeführt. Bei der Entscheidung über deren Umstellung wurde ohne klinischen Anhalt auf eine Gerinnungsneigung von Seiten der Klinik nicht eingegriffen. Stattdessen wurden die vom Hausarzt, Patienten oder niedergelassenen Ophthalmologen vorgenommenen Umstellungen der Medikation übernommen oder die Medikation belassen. So konnten zudem im Rahmen der Studie besonders praxisnahe Daten erhoben werden.

Auch innerhalb der Substanzgruppen das jeweils gewählte Vorgehen uneinheitlich: TFH wurden von 70 (73,7\%) Patienten perioperativ weiterhin eingenommen, in 25 (26, 3\%) Fällen wurden diese hingegen präoperativ abgesetzt. Von den 14 Patienten, mit einer dauerhaften Phenprocoumon-Einnahme, wurde die Medikation präoperativ bei vier Patienten (28,5\%) nicht verändert, sechs (42,9\%) Patienten erhielten ein Bridging mit Heparin, bei vier $(28,5 \%)$ 
Patienten wurde die Medikation perioperativ abgesetzt. Die Medikation von NOAK wurde bei fünf (21,7\%) Patienten perioperativ nicht verändert, bei einem (4,3\%) Patienten perioperativ durch Heparin ersetzt, bei 17 (73,9\%) Patienten wurde sie abgesetzt. Zudem folgte die jeweilige Anordnung nicht einheitlich einer Empfehlung des behandelnden Augenchirurgen, sondern wurde häufig vom Hausarzt oder dem Patienten selbst entschieden. Eine Übersicht der präoperativen Veränderung der Gerinnungsmedikation ist in der Tabelle $6 \mathrm{zu}$ finden. ${ }^{3}$

Tabelle 6: Veränderung der Medikation bei Patienten mit einer Netzhautoperation

\begin{tabular}{|c|c|c|c|c|}
\hline \multirow[b]{2}{*}{ Präparat } & \multicolumn{3}{|c|}{ (Art der) Veränderung der Medikation } & \multirow[b]{2}{*}{ Total } \\
\hline & nein & Bridging & Absetzen & \\
\hline TFH: ASS & $64(74,4 \%)$ & $0(0 \%)$ & $22(25,6 \%)$ & 86 \\
\hline TFH: Clopidogrel & $4(57,1 \%)$ & $0(0 \%)$ & $3(42.9 \%)$ & 7 \\
\hline TFH: Prasugrel & $1(100 \%)$ & $0(0 \%)$ & $0(0 \%)$ & 1 \\
\hline TFH: Ticagrelor & $1(100 \%)$ & $0(0 \%)$ & $0(0 \%)$ & 1 \\
\hline VKA: Phenprocoumon & $4(28,6 \%)$ & $6(42,9 \%)$ & $4(28,6 \%)$ & 14 \\
\hline NOAK: Dabigatran & 0 & 0 & 0 & 0 \\
\hline NOAK: Apixaban & $4(26,7 \%)$ & $1(6,7 \%)$ & $10(66,7 \%)$ & 15 \\
\hline NOAK: Rivaroxaban & $0(0 \%)$ & $0(0 \%)$ & $5(100 \%)$ & 5 \\
\hline NOAK: Edoxaban & $1(33,3 \%)$ & $0(0 \%)$ & $2(66,7 \%)$ & 3 \\
\hline Nicht-orale AK: Heparin- $\mathrm{Na} /$ Heparin-Ca & $1(100 \%)$ & $0(0 \%)$ & $0(0 \%)$ & 1 \\
\hline Nem: Knoblauch & $0(0 \%)$ & $0(0 \%)$ & $0(0 \%)$ & 0 \\
\hline Nem: Ginkgo & $1(50 \%)$ & $0(0 \%)$ & $1(50 \%)$ & 2 \\
\hline Nem: Padma 28 & $1(100 \%)$ & $0(0 \%)$ & $0(0 \%)$ & 1 \\
\hline Total & 82 & 7 & 47 & 136 \\
\hline
\end{tabular}

${ }^{3}$ Die Differenz der Anzahl von Patienten mit Gerinnungshemmern und eingenommenen Medikamenten in dieser Tabelle ist dadurch bedingt, dass es durch Kombination mehrerer Gerinnungshemmer, wie beispielsweise bei einer dualen Plättchenhemmung, mehr verordnete Medikamente als Patienten, die Gerinnunghemmer verwenden, gibt. 


\subsubsection{Anästhesieformen}

Bei den in der Studie durchgeführten Eingriffen wurden unterschiedliche Anästhesieverfahren angewandt. Eine Intubationsnarkose wurde bei 239 (63,9\%) der Netzhauteingriffe durchgeführt, 129 (34,5\%) der Patienten wurden unter einer Retrobulbäranästhesie operiert, bei zwei $(0,5 \%)$ Operationen wurde über betäubende Augentropfen und in einer $(0,3 \%)$ wurde mit einer Kombination aus Tropfen und Retrobulbäranästhesie betäubt.

\subsubsection{Eingriff}

Bei den vorgenommenen Operationen handelte es sich bei 244 (65,2\%) Patienten um einen Ersteingriff, bei 130 (34,8\%) Patienten wurde eine Wiederholungsoperation durchgeführt. Häufigste Netzhautoperation war die Pars-Plana-Vitrektomie bei epiretinaler Gliose, bei 73 $(19,5 \%)$ der Patienten, gefolgt von einer ppV bei Makulaforamen (62 Patienten/16,6\%) und ppV bei Netzhautablösung (59 Patienten/15,7\%). Alle durchgeführten Eingriffe sind zusammengefasst in der Abbildung 7 dargestellt und weiter unten in Tabelle 18 (Seite 47) nochmals nach einzelnen Häufigkeiten aufgeführt. 


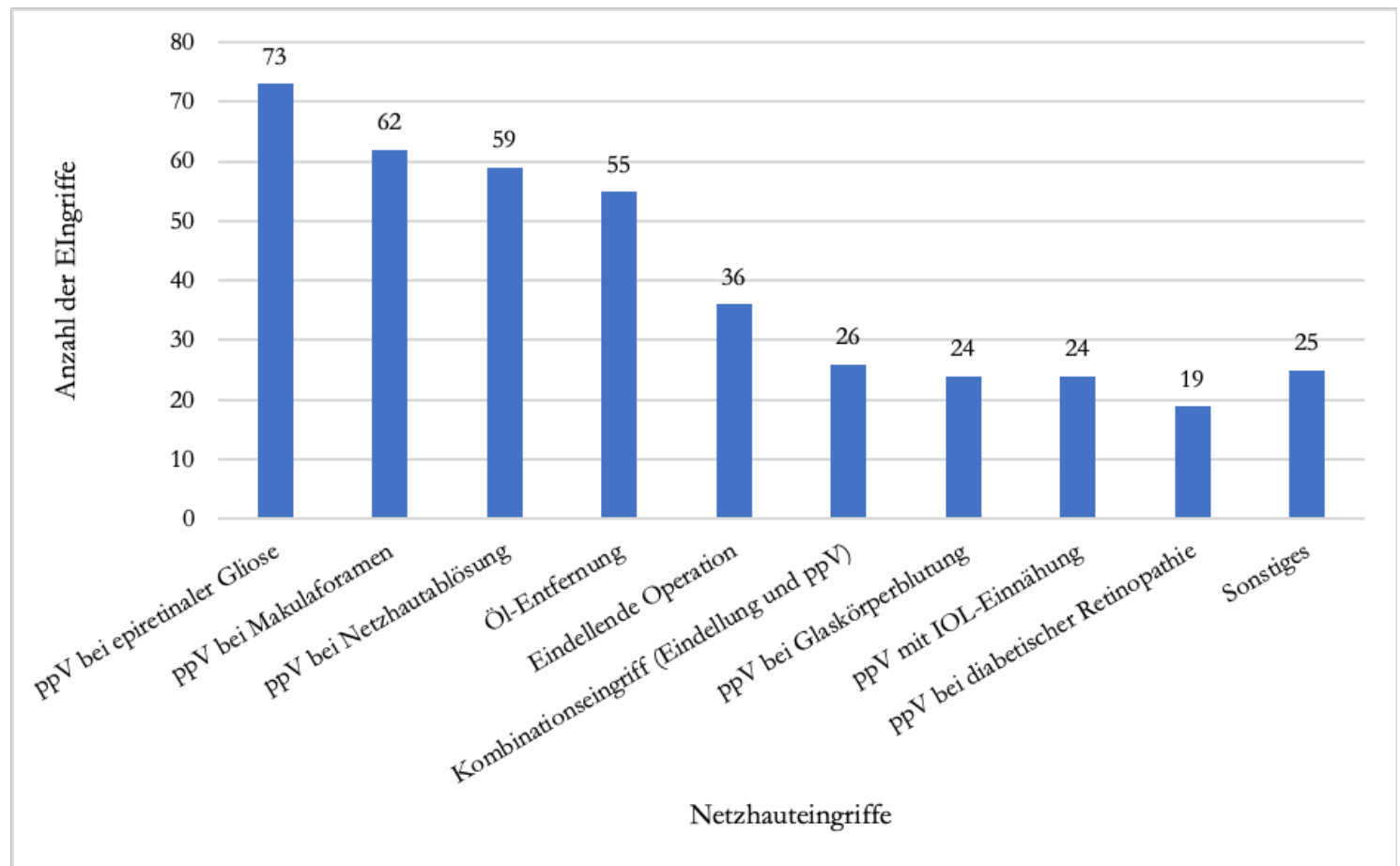

Abbildung 7: Verteilung der Netzhautoperationen ( $\mathrm{n}=403$, Mehrfachnennungen möglich)

\subsubsection{Eingriffsdauer}

Die Netzhauteingriffe dauerten zwischen neun und 123 Minuten (Median 36 Minuten (Q1:29, Q3:50)). Die Eingriffsdauer der Wiederholungseingriffe war dabei signifikant länger als bei Ersteingriffen $(\mathrm{p}$-Wert $=0,005)$. 


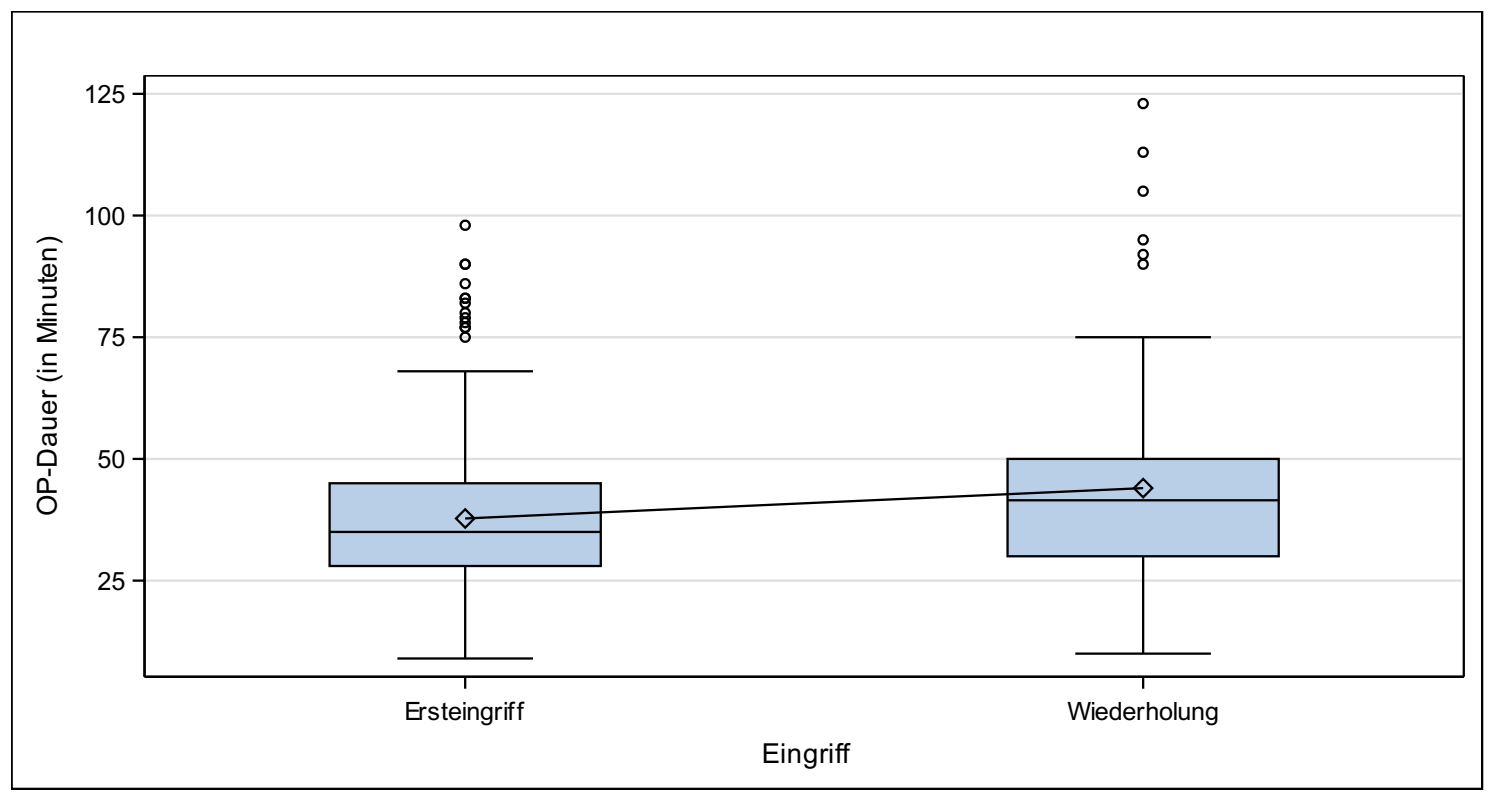

Abbildung 8: OP-Dauer bei Erst- und Wiederholungseingriffen bei Netzhautoperationen (Ersteingriff: $\mathrm{n}=244$, Wiederholungseingriff: $\mathrm{n}=130$ )

\subsubsection{Perioperative Blutdruckmessung}

Im Rahmen der Studie wurden intraoperativ der Anfangs- und End- sowie der minimale und maximale Blutdruckwert jeweils sowohl systolisch als auch diastolisch erhoben. Zusätzlich wurde bei allen Patienten der postoperative Blutdruck im Aufwachraum gemessen. Da dieser Blutdruckwert auf den postoperativen Bögen der Patienten mit Lokalanästhesie oftmals fehlte, wurde in solchen Fällen stattdessen der intraoperative Endblutdruckwert verwendet. Hierbei lag die Annahme zugrunde, dass sich der postoperative Blutdruckwert nach einer Lokalanästhesie nicht stark verändern und insbesondere im Vergleich zum intraoperativen Endwert nicht wesentlich ansteigen würde, da die Patienten nicht dem intraoperativ zu beobachtenden blutdrucksenkendem Effekt einer Allgemeinanästhesie ausgesetzt waren.

Der mittlere erhobene maximale intraoperative Blutdruckwert bei Netzhautpatienten (Median) lag systolisch bei 152,5 $\mathrm{mmHg}$, wobei das untere Quartil bei 133,5 $\mathrm{mmHg}$ und das obere bei $170 \mathrm{mmHg}$ lag. Diastolisch reichten die maximalen Blutdruckwerte im ersten Quartil bis $60 \mathrm{mmHg}$, im dritten bis $80 \mathrm{mmHg}$, und der Median lag bei $70 \mathrm{mmHg}$. Postoperativ lagen die systolischen Blutdruckwerte zwischen einem Q1 von $130 \mathrm{mmHg}$ und einem Q3 von 160 mmHg (diastolisch: Q1:61 mmHg, Q3:80 mmHg) mit einem Median von 
$142 \mathrm{mmHg}$ (diastolisch: $70 \mathrm{mmHg}$ ). Alle Werte sind aus Gründen der Übersichtlichkeit in der Tabelle 7 aufgelistet. ${ }^{4}$

Tabelle 7: Perioperative Blutdruckwerte der Netzhautpatienten

\begin{tabular}{|c|c|c|c|c|c|c|c|c|c|}
\hline Messung & Gruppe & $\mathbf{N}$ & Mean & STD & Min & Q1 & Median & Q3 & Max \\
\hline Anfang & systolisch & 332 & 151,2 & 25,0 & 93,0 & 132,0 & 150,0 & 170,0 & 226,0 \\
\hline$[\mathrm{mmHg}]$ & diastolisch & 332 & 72,7 & 14,5 & 30,0 & 60,0 & 70,0 & 80,0 & 120,0 \\
\hline Ende & systolisch & 332 & 121,8 & 22,9 & 75,0 & 105,0 & 115,5 & 135,5 & 198,0 \\
\hline$[\mathrm{mmHg}]$ & diastolisch & 332 & 60,4 & 13,8 & 28,0 & 50,0 & 60,0 & 70,0 & 140,0 \\
\hline Min & systolisch & 332 & 108,1 & 27,5 & 51,0 & 90,0 & 100,0 & 130,0 & 190,0 \\
\hline$[\mathrm{mmHg}]$ & diastolisch & 332 & 54,4 & 15,6 & 20,0 & 40,0 & 50,0 & 61,0 & 120,0 \\
\hline Max & systolisch & 332 & 152,5 & 27,4 & 70,0 & 133,5 & 152,5 & 170,0 & 246,0 \\
\hline [mmHg] & diastolisch & 332 & 72,6 & 15,4 & 30,0 & 60,0 & 70,0 & 80,0 & 140,0 \\
\hline Postop & systolisch & 331 & 145,2 & 21,8 & 90,0 & 130,0 & 142,0 & 160,0 & 215,0 \\
\hline$[\mathrm{mmHg}]$ & diastolisch & 331 & 71,8 & 13,5 & 25,0 & 61,0 & 70,0 & 80,0 & 140,0 \\
\hline
\end{tabular}

\subsubsection{Relevante perioperative Blutungskomplikationen bei Netzhauteingriffen}

Die Anzahl relevanter intraoperativer Blutungen (im Sinne der für diese Arbeit gewählten Definition, vgl. 2.4.3) bei Netzhautpatienten lag bei 13 (3,5\%). Neun Blutungen der Stufe 3 gingen von der Netzhaut aus, drei Blutungen der Stufe 3 und eine Blutung der Stufe 4 gingen von der Aderhaut aus. Kammerwinkel/Iris und Sklera/Ziliarkörper spielten als Blutungsquelle relevanter Blutungen keine Rolle. Eine Operation musste aufgrund einer Blutungskomplikation bei der Retrobulbäranästhesie abgebrochen werden. Zur Übersicht sind alle intraoperativen Blutungen bei Netzhautoperationen in der Tabelle 8 aufgeführt.

${ }^{4}$ Der Differenz zwischen den in dieser Tabelle ausgewerteten Blutdruckwerten von 332 Patienten und der Größe des Gesamtkollektivs von 374 Patienten, liegt die Tatsache zugrunde, dass perioperativ nicht von allen Patienten Blutdruckwerte aufgeschrieben wurden und bei Patienten mit einer Lokalanästhesie die Blutdruckwerte postoperativ nicht nachvollzogen werden konnten. 
Tabelle 8: Intraoperative Blutungen bei Netzhautoperationen

\begin{tabular}{|c|c|c|c|c|c|}
\hline & & Blutungs & quelle & & \\
\hline $\begin{array}{c}\text { Blutung im } \\
\text { Blutungsgrading }\end{array}$ & Netzhaut & $\begin{array}{c}\text { Kammerwinkel/ } \\
\text { Iris }\end{array}$ & $\begin{array}{c}\text { Sklera/ } \\
\text { Ziliarkörper }\end{array}$ & Aderhaut & Insgesamt \\
\hline 1 & $23(6,2 \%)$ & 0 & $6(1,6 \%)$ & $1(0,3 \%)$ & 30 \\
\hline 2 & $3(0,8 \%)$ & 0 & $3(0,8 \%)$ & $2(0,5 \%)$ & 8 \\
\hline 3 & $9(2,4 \%)$ & 0 & 0 & $3(0,8 \%)$ & 12 \\
\hline 4 & 0 & 0 & 0 & $1(0,3 \%)$ & 1 \\
\hline 5 & 0 & 0 & 0 & 0 & 0 \\
\hline Total & 35 & 0 & 9 & 7 & 51 \\
\hline
\end{tabular}

Die angegebenen Prozentzahlen stellen den Anteil an allen Patienten mit einem Netzhauteingriff dar.

Eine dauerhaft visusbedrohende Blutung trat bei zwei $(2,2 \%)$ Netzhautpatienten auf, in einem dieser beiden Fällen wurde aufgrund der Blutungskomplikation auch eine ReOperation erforderlich. Beide Re-Operationen waren nicht direkte Folge einer intraoperativen Blutung. Eine der Re-Operationen wurde infolge einer Nachblutung erforderlich, die andere aufgrund einer starken Blutung bei der Retrobulbäranästhesie. Insgesamt traten also bei $15(4,0 \%)$ der Patienten mit Netzhautoperationen eine relevante Blutungskomplikation auf.

Eine Nachblutung trat bei 60 (16,1\%) Patienten auf. Die häufigste Quelle der Nachblutungen war die Bindehaut. Die weitere Verteilung der Nachblutungsquellen ist in der nachfolgenden Abbildung 9 dargestellt. 


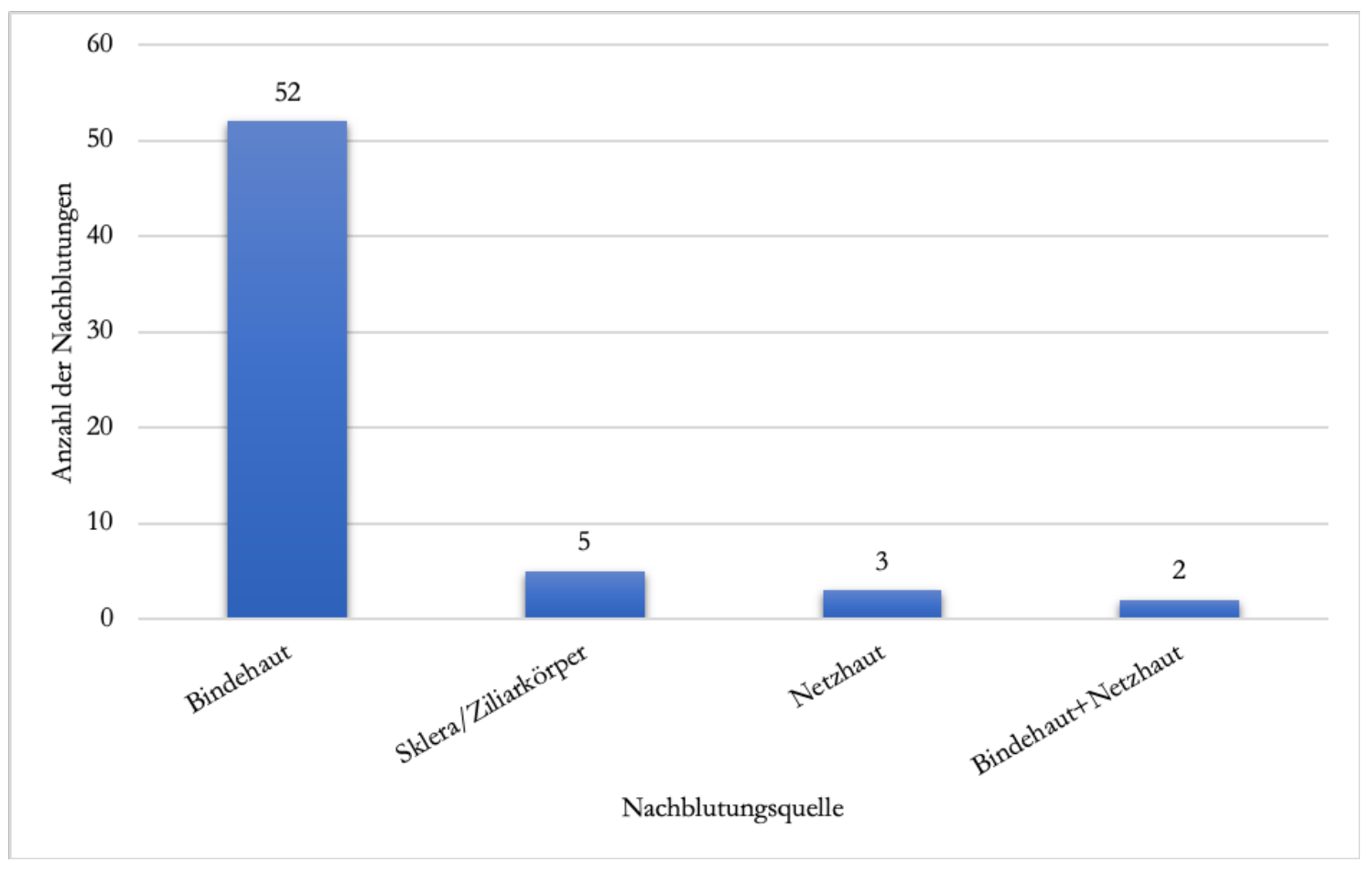

Abbildung 9: Nachblutungen bei Netzhautoperationen

\subsection{Glaukompatienten}

$49(55,1 \%)$ der Glaukompatienten waren weiblich, 40 (44,9\%) männlich. 45 Mal (50,6\%) wurde das rechte Auge operiert, $44 \mathrm{Mal}(49,4 \%)$ das linke Auge. Das Alter der Patienten zum Zeitpunkt der Operation reichte von 38 bis 91 Jahren. Im Mittel waren die Patienten 71,3 Jahre alt, die Standardabweichung betrug 10,5 Jahre.

\subsubsection{Kardiovaskuläre Begleiterkrankungen}

Bei 69 (77,5\%) der Patienten lagen kardiovaskuläre Begleiterkrankungen vor. Dabei entfiel bei diesem Kollektiv der größte Anteil mit 59 (66,29\%) Betroffenen auf die arterielle Hypertonie, gefolgt von Diabetes mellitus (12 Erkrankte/13,48\%) und Tiefer Beinvenenthrombose (10/11,24\%). Alle relevanten Begleiterkrankungen der Patienten mit Glaukomoperation sind zusammengefasst in der Abbildung 10 sowie in Tabelle 21 auf Seite 49 dargestellt. 


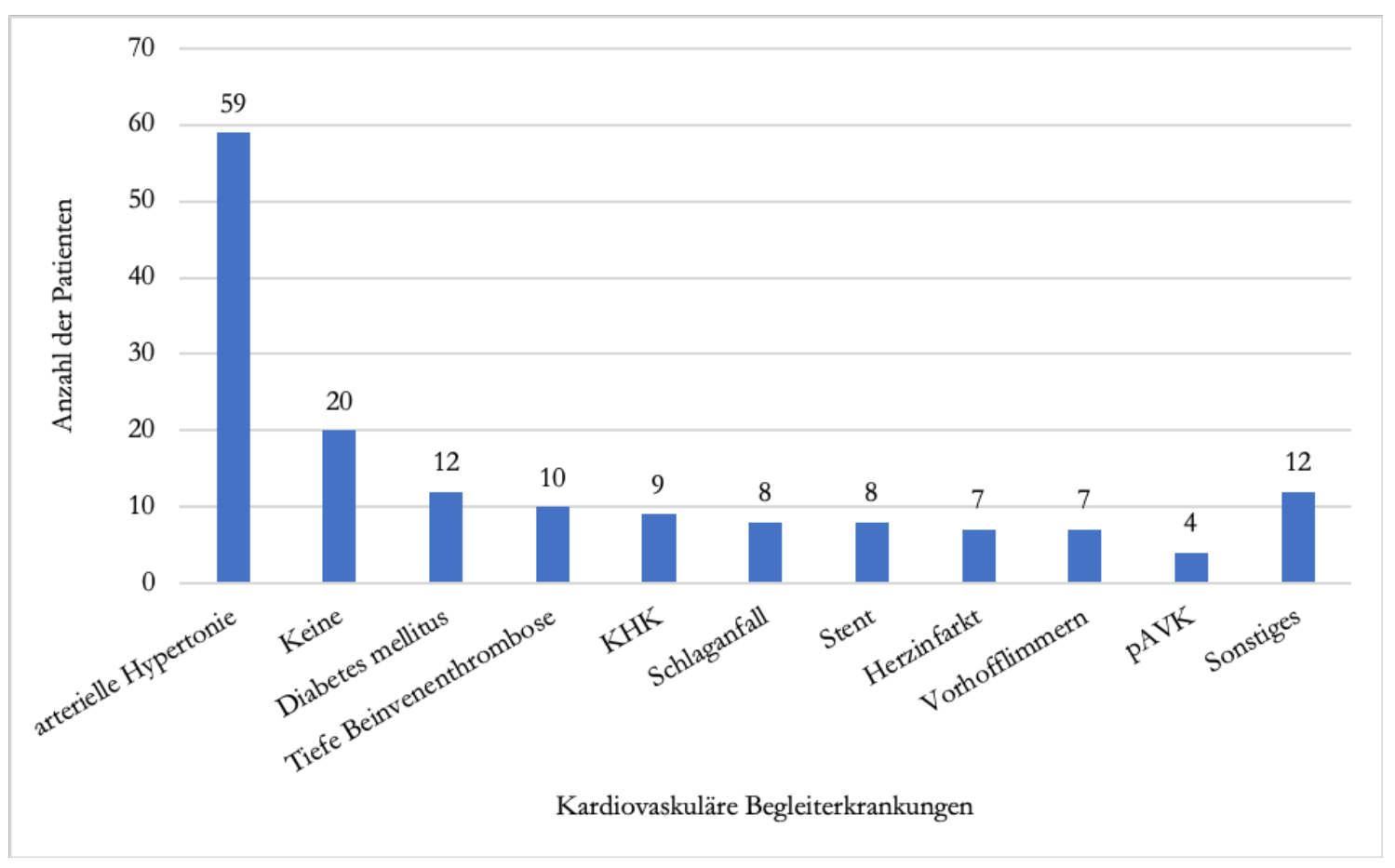

Abbildung 10: Begleiterkrankungen bei Patienten mit Glaukomoperationen ( $\mathrm{n}=156$,

Mehrfachnennungen möglich)

\subsubsection{Einnahme gerinnungsmodifizierender Medikamente}

Gerinnungsmodulierende Medikamente wurden von 32 (36\%) der Glaukompatienten zum Zeitpunkt der Operation eingenommen. Davon erhielten 17 (53,1\%) den Thrombozytenaggregationshemmer ASS und 5 (15,6\%) Clopidogrel; 57 (64\%) Patienten nahmen keine gerinnungsbeeinflussenden Substanzen ein. Auch bei den Glaukomoperationen war der präoperative Umgang mit der gerinnungshemmenden Medikation nicht einheitlich: Bei 12 (52,2\%) der Patienten mit TFH wurde die Medikation beibehalten, ein (4,3\%) Patient erhielt eine perioperative Überbrückung mit Heparin, bei 10 (43,5\%) Patienten wurden die TFH perioperativ abgesetzt. Von den beiden mit Phenprocoumon vorbehandelten Patienten erhielt einer ein Bridging, bei dem anderen wurde das Medikament perioperativ ohne Bridging abgesetzt. Bei allen sechs Patienten mit NOAK wurde deren Einnahme perioperativ ausgesetzt. Eine Übersicht aller eingenommenen gerinnungsbeeinflussenden Substanzen und des perioperativen Umgangs mit diesen ist in der Tabelle 9 dargestellt. ${ }^{5}$

5 Die auch in dieser Tabelle vorkommende Differenz zwischen der Anzahl von Patienten mit Gerinnungshemmern (32) und eingenommenen Medikamenten (36) wurde bereits zuvor erläutert (vgl. Fußnote 3) 
Tabelle 9: Veränderung der Medikation bei Patienten mit Glaukomoperationen

\begin{tabular}{|c|c|c|c|c|}
\hline \multirow[b]{2}{*}{ Präparat } & \multicolumn{3}{|c|}{ (Art der) Veränderung der Medikation } & \multirow[b]{2}{*}{ Total } \\
\hline & nein & Bridging & Absetzen & \\
\hline TFH: ASS & $10(58.8 \%)$ & $0(0 \%)$ & $7(41,2 \%)$ & 17 \\
\hline TFH: Clopidogrel & $1(20 \%)$ & $1(20 \%)$ & $3(60 \%)$ & 5 \\
\hline TFH: Prasugrel & 0 & 0 & 0 & 0 \\
\hline TFH: Ticagrelor & $1(100 \%)$ & $0(0 \%)$ & $0(0 \%)$ & 1 \\
\hline VKA: Phenprocoumon & $0(0 \%)$ & $1(50 \%)$ & $1(50 \%)$ & 2 \\
\hline NOAK: Dabigatran & $0(0 \%)$ & $0(0 \%)$ & $2(100 \%)$ & 2 \\
\hline NOAK: Apixaban & $0(0 \%)$ & $0(0 \%)$ & $1(100 \%)$ & 1 \\
\hline NOAK: Rivaroxaban & $0(0 \%)$ & $0(0 \%)$ & $2(100 \%)$ & 2 \\
\hline NOAK: Edoxaban & $0(0 \%)$ & $0(0 \%)$ & $1(100 \%)$ & 1 \\
\hline Nicht-orale AK: Heparin- $\mathrm{Na} /$ Heparin-Ca & 0 & 0 & 0 & 0 \\
\hline Nem: Knoblauch & $0(0 \%)$ & $0(0 \%)$ & $1(100 \%)$ & 1 \\
\hline Nem: Ginkgo & $1(25 \%)$ & $0(0 \%)$ & $3(75 \%)$ & 4 \\
\hline Nem: Padma 28 & 0 & 0 & 0 & 0 \\
\hline Total & 13 & 2 & 21 & 36 \\
\hline
\end{tabular}

\subsubsection{Anästhesieformen}

Auch bei den Glaukomoperationen kamen unterschiedliche Formen der Anästhesie zum Einsatz. Bei $62(69,7 \%)$ Patienten wurde eine Intubationsanästhesie durchgeführt, 26 $(29,2 \%)$ erhielten eine Anästhesie über betäubende Augentropfen und ein (1,1\%) Patient wurde unter einer parabulbären Anästhesie operiert.

\subsubsection{Eingriff}

60 (67,4\%) Patienten wurden zum ersten Mal wegen des jeweiligen Krankheitsbildes operiert, bei 29 Patienten (32,6\%) war es ein Wiederholungseingriff. Der am häufigsten durchgeführte Glaukomeingriff war die Trabekulotomie mit dem Trabektom, welche bei 38 (42,7\%) Patienten durchgeführt wurde. Eine Trabekulektomie erfolgte bei 30 (33,7\%) Patienten, eine Kanaloplastik bei neun (10,1\%), Kahook bei sechs (6,7\%) und die Implantation eines Ahmed- oder Baerveldt-Ventils ebenfalls bei sechs (6,74\%) Patienten. Eine Übersicht aller 
durchgeführten Glaukomeingriffe ist in der nachfolgenden Abbildung 11 sowie in der Tabelle 22 auf Seite 50 zu finden.

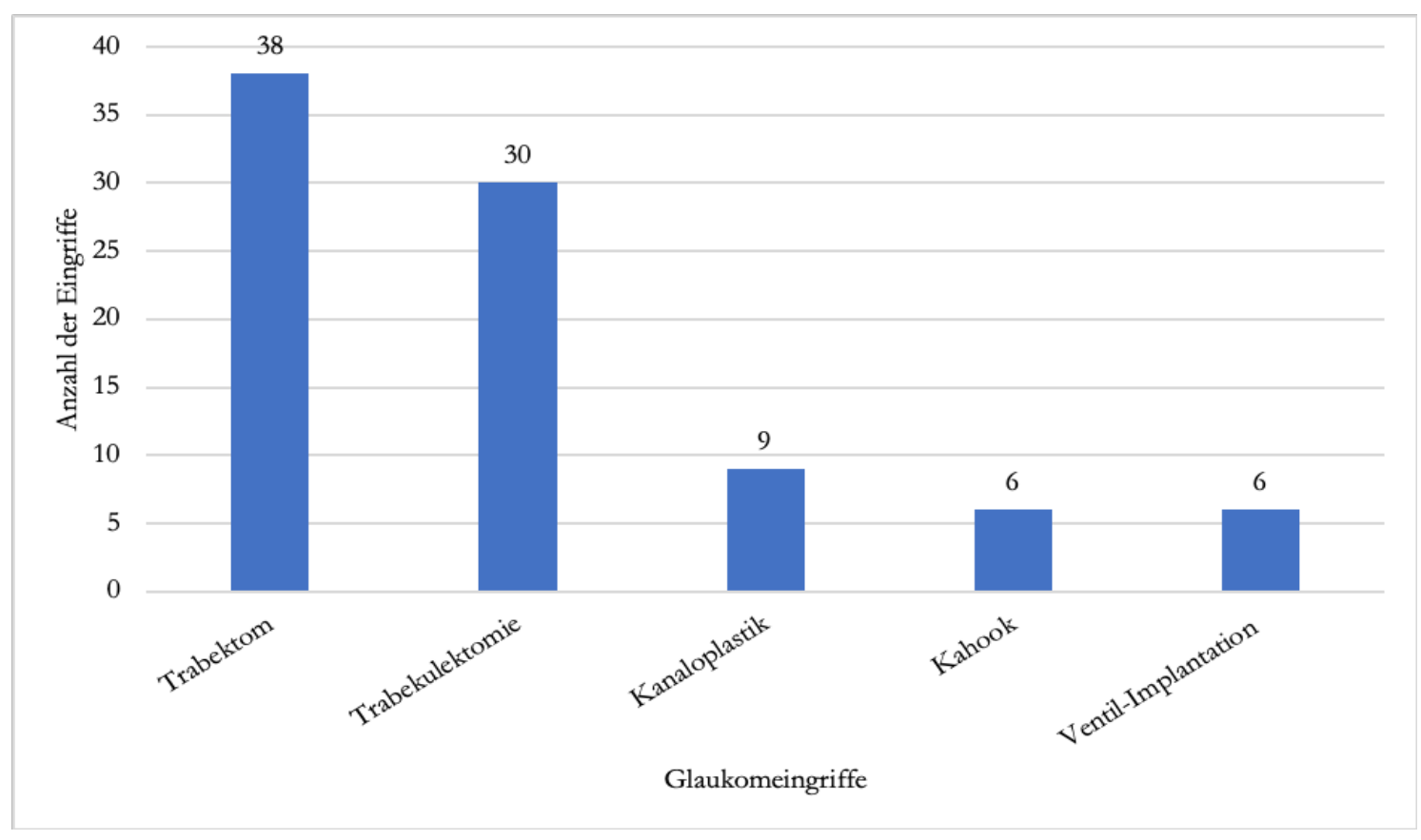

Abbildung 11: Verteilung der Glaukomoperationen $(\mathrm{n}=89)$

Die Dauer der Glaukomoperationen betrug 7 bis 94 Minuten, mit einem mittleren Wert (Median) von 35 Minuten, einem ersten Quartilswert von 17 und einem oberen Quartil von 49 Minuten.

\subsubsection{Perioperative Blutdruckmessung}

Bei den Glaukomoperationen wurden in gleicher Weise wie bei den Netzhautoperationen vier intraoperative sowie ein postoperativer sowohl systolischer als auch diastolischer Blutdruckwert erhoben. Der Median der intraoperativen maximalen systolischen Blutdruckwerte betrug $153 \mathrm{mmHg}$, das untere Quartil $136 \mathrm{mmHg}$ und das obere Quartil $165 \mathrm{mmHg}$, diastolisch $70 \mathrm{mmHg}$ (Q1: $60 \mathrm{mmHg}$, Q3: $80 \mathrm{mmHg}$ ). Postoperativ lag der mittlere Blutdruckwert systolisch bei $140 \mathrm{mmHg}$ (Q1:130 mmHg, Q3: $157 \mathrm{mmHg}$ ), diastolisch bei $70 \mathrm{mmHg}(\mathrm{Q} 1: 60 \mathrm{mmHg}, \mathrm{Q} 3: 80 \mathrm{mmHg}$ ). Alle erhobenen Blutdruckwerte bei Glaukomoperationen sind in Tabelle 10 aufgezeigt. ${ }^{6}$

${ }_{6}$ Auch in dieser Tabelle ist die Differenz von 76-71 ausgewerteten Blutdruckwerten im Gegensatz zu 89 Glaukompatienten durch fehlende Daten bei der Erhebung zu erklären. (Vgl. Fußnote 4) 
Tabelle 10: Perioperative Blutdruckwerte der Glaukompatienten

\begin{tabular}{|l|l|r|r|r|r|r|r|r|r|}
\hline Messung & Gruppe & \multicolumn{1}{|l}{ N } & \multicolumn{1}{|l}{ Mean } & \multicolumn{1}{c|}{ STD } & \multicolumn{1}{c|}{ Min } & Q1 & Median & Q3 & Max \\
\hline Anfang & systolisch & 76 & 152,3 & 24,4 & 100,0 & 137,0 & 150,0 & 167,5 & 240,0 \\
\hline [mmHg] & diastolisch & 76 & 71,7 & 13,2 & 40,0 & 60,0 & 70,0 & 80,0 & 100,0 \\
\hline Ende & systolisch & 73 & 112,9 & 20,4 & 85,0 & 100,0 & 110,0 & 120,0 & 176,0 \\
\hline [mmHg] & diastolisch & 73 & 60,4 & 13,8 & 28,0 & 50,0 & 60,0 & 70,0 & 140,0 \\
\hline Min & systolisch & 73 & 101,8 & 27,1 & 60,0 & 82,0 & 90,0 & 110,0 & 180,0 \\
\hline [mmHg] & diastolisch & 73 & 54,4 & 15,6 & 20,0 & 40,0 & 50,0 & 61,0 & 120,0 \\
\hline Max & systolisch & 74 & 151,5 & 28,0 & 80,0 & 136,0 & 153,0 & 165,0 & 240,0 \\
\hline [mmHg] & diastolisch & 74 & 71,7 & 13,8 & 40,0 & 60,0 & 70,0 & 80,0 & 100,0 \\
\hline Postop & systolisch & 71 & 143,0 & 20,3 & 100,0 & 130,0 & 140,0 & 157,0 & 187,0 \\
\hline [mmHg] & diastolisch & 71 & 71,2 & 10,2 & 50,0 & 60,0 & 70,0 & 80,0 & 100,0 \\
\hline
\end{tabular}

\subsubsection{Relevante perioperative Blutungskomplikation bei Glaukomeingriffen}

Relevante perioperative Blutungskomplikationen traten bei $8(9 \%)$ Glaukompatienten auf. Eine Blutung der Stufe 3 ging von der Bindehaut aus, zwei vom Kammerwinkel/der Iris. Sechs Blutungen kamen von der Sklera/dem Kammerwinkel, davon gab es fünf Blutungen der Stufe 3 und eine der Stufe 4. Eine Blutung der Aderhaut führte aufgrund der Stärke der Blutung zum Abbruch des Eingriffs (Stufe 5). Eine tabellarische Darstellung von Blutungen und Blutungsquellen ist in Tabelle $11 \mathrm{zu}$ sehen $^{7}$. Es trat eine (1,1\%) Blutung auf, die als dauerhaft visusbedrohend bewertet wurde und eine Re-Operation erforderlich machte.

7 Aufgrund von mehrfachen Blutungsquellen und -ereignissen bei einigen Patienten übersteigt die Gesamtzahl der Blutungsereignisse (130) die Anzahl der Glaukompatienten (89). 
Tabelle 11: Intraoperative Blutungen bei Glaukompatienten

\begin{tabular}{|c|c|c|c|c|c|}
\hline & & Blutung & squelle & & \\
\hline $\begin{array}{c}\text { Stärke der } \\
\text { Blutung im } \\
\text { Blutungsgrading }\end{array}$ & Bindehaut & $\begin{array}{c}\text { Kammerwinkel/ } \\
\text { Iris }\end{array}$ & $\begin{array}{c}\text { Sklera/ } \\
\text { Ziliarkörper }\end{array}$ & Aderhaut & Insgesamt \\
\hline 1 & $44(50 \%)$ & $30(33,7 \%)$ & $24(27 \%)$ & $1(1,1 \%)$ & 99 \\
\hline 2 & $6(6,8 \%)$ & $11(12,4 \%)$ & $4(4,5 \%)$ & 0 & 21 \\
\hline 3 & $1(1,1 \%)$ & $2(2,2 \%)$ & $5(5,6 \%)$ & 0 & 8 \\
\hline 4 & 0 & 0 & $1(1,1 \%)$ & 0 & 1 \\
\hline 5 & 0 & 0 & 0 & $1(1,1 \%)$ & 1 \\
\hline Total & 51 & 43 & 34 & 2 & 130 \\
\hline
\end{tabular}

Die angegebenen Prozentzahlen stellen den Anteil an allen Patienten mit einem Glaukomeingriff dar.

15 (16,9\%) Patienten hatten eine Nachblutung. Die häufigste Quelle von Nachblutungen bei Glaukomoperationen war, bei acht (53,3\%) Patienten, die Sklera/der Ziliarkörper, gefolgt von $40 \%$ Bindehaut- und 6,7\% Aderhautnachblutungen. Alle Nachblutungsquellen bei Patienten mit einer Glaukomoperation sind in der Abbildung 12 dargestellt.

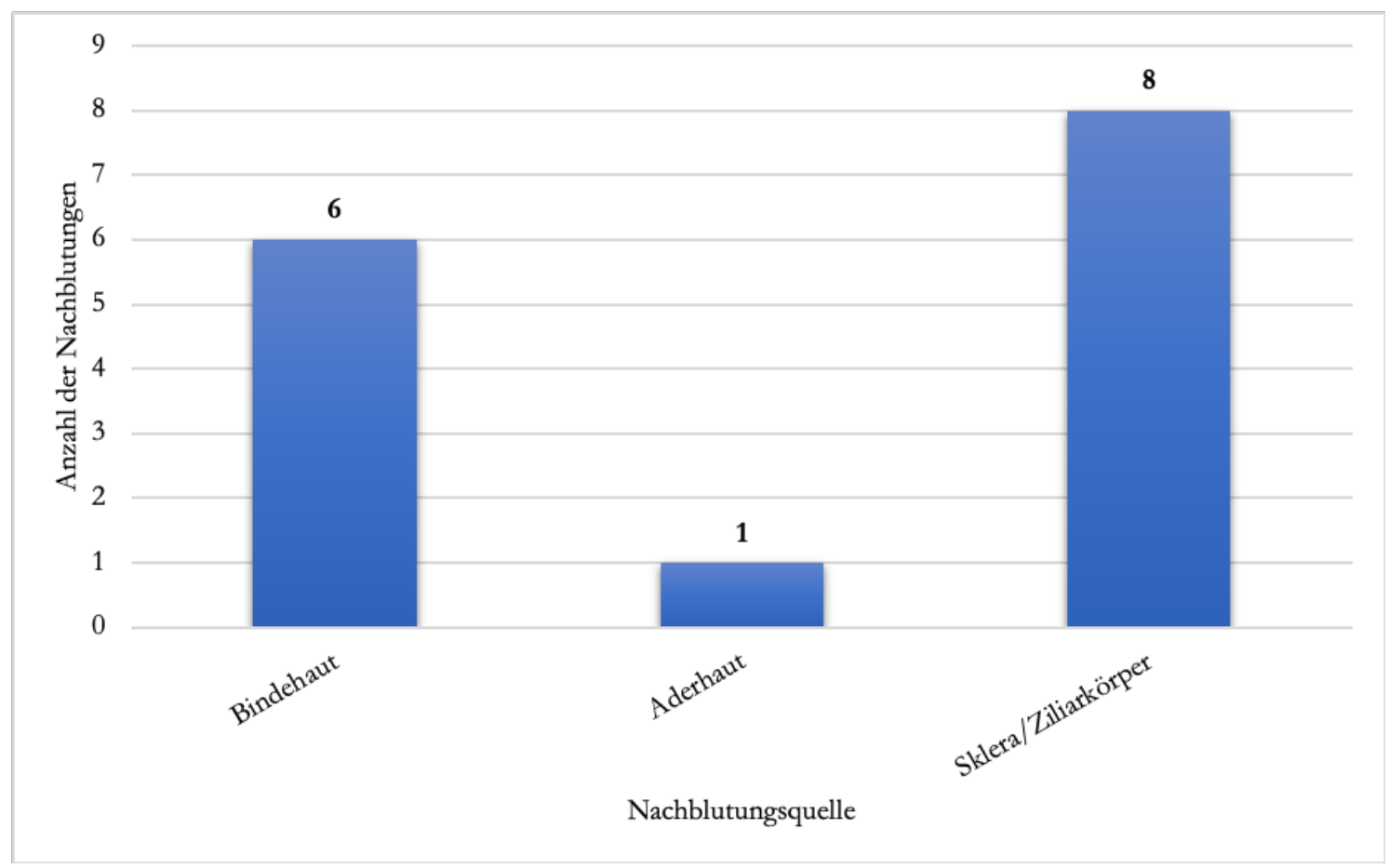

Abbildung 12: Nachblutungen bei Glaukomoperationen $(\mathrm{n}=15)$ 
Die Verteilung aller aufgetretenen relevanten Blutungskomplikationen bei Netzhaut- und Glaukompatienten ist nachfolgend in der Tabelle 12 dargestellt.

Tabelle 12: Verteilung relevanter Blutungskomplikationen

\begin{tabular}{|c|c|c|c|c|}
\hline & \multicolumn{3}{|c|}{ Relevante Blutungskomplikationen } & \multirow[b]{2}{*}{ Insgesamt } \\
\hline & $\begin{array}{l}\text { Blutung ab Stärke } 3 \\
\text { im Blutungsgrading }\end{array}$ & Re-Operation nötig & $\begin{array}{c}\text { dauerhafte } \\
\text { Visusbedrohung }\end{array}$ & \\
\hline Netzhautoperationen & 13 & 2 & 2 & 15 \\
\hline Glaukomoperationen & 8 & 1 & 1 & 8 \\
\hline
\end{tabular}

\subsection{Mögliche Zusammenhänge und Risikofaktoren}

Insgesamt war zu überprüfen, ob es bei allen Patienten, bei denen eine relevante perioperative Blutung auftrat, gemeinsame Faktoren gab, die auf eine erhöhtes intraoperatives Blutungsrisiko hindeuten könnten. Dabei werden im Folgenden zunächst diejenigen Zusammenhänge dargestellt, die sich in beiden Gruppen fanden. Im Anschluss werden die Ergebnisse vorgestellt, in denen sich die Kollektive unterschieden. Nachfolgend werden relevante Blutungskomplikationen und erhobene Merkmale auf statistische Zusammenhänge untersucht, um mögliche Risikofaktoren zu identifizieren.

\subsubsection{Mögliche übereinstimmende Risikofaktoren bei Netzhaut- und}

\section{Glaukomoperationen}

Die Häufigkeit relevanter Blutungskomplikationen war nicht seitendifferent. Bei Glaukomeingriffen traten häufiger Blutungskomplikationen auf als bei Netzhautoperationen, dieser Unterschied war jedoch nicht statistisch signifikant (Tabelle 13).

Tabelle 13: Blutungskomplikationen bei Netzhaut- und Glaukomoperationen

\begin{tabular}{|c|c|c|c|c|c|c|c|}
\hline \multirow[b]{2}{*}{ Gruppe } & \multicolumn{2}{|c|}{$\begin{array}{l}\text { Relevante } \\
\text { Blutungskomplikation }\end{array}$} & \multirow[b]{2}{*}{ Total } & \multicolumn{3}{|c|}{ Chi square test } & \multirow{2}{*}{$\begin{array}{l}\text { Fisher's } \\
\text { exact test } \\
\text { p-value }\end{array}$} \\
\hline & ja & nein & & DF & $\begin{array}{l}\text { Test- } \\
\text { statistic }\end{array}$ & p-value & \\
\hline Glaukom & $8(9 \%)$ & $81(91 \%)$ & $89(19,2 \%)$ & 1 & 3,7738 & 0,0521 & 0,0604 \\
\hline Netzhaut & $15(4 \%)$ & $359(96 \%)$ & $374(80,8 \%)$ & . & . & . & \\
\hline Total & $23(5 \%)$ & $440(95 \%)$ & $463(100 \%)$ & & & . & \\
\hline
\end{tabular}


Auch zwischen Ersteingriffen und Wiederholungsoperationen und zwischen unterschiedlichen Anästhesie- bzw. Narkoseverfahren (

Tabelle 14) fanden sich keine signifikanten Unterschiede der Häufigkeit relevanter Blutungskomplikationen (alle p-Werte über dem Signifikanzniveau von 0,05). Auch die Tatsache, ob es sich um einen Ersteingriff oder eine Wiederholungsoperation handelte, zeigte keinen statistischen Zusammenhang.

Tabelle 14: Blutungskomplikationen und Anästhesieverfahren bei Netzhaut- und

\section{Glaukomoperationen}

\begin{tabular}{|c|c|c|c|c|c|c|}
\hline & & Anästhesi & erfahren & & & $\begin{array}{l}\text { Fisher's } \\
\text { exact test }\end{array}$ \\
\hline $\begin{array}{l}\text { Relevante } \\
\text { Blutungs- } \\
\text { komplikation }\end{array}$ & Tropf & Retrobulbär & Parabulbär & ITN & Total & p-value \\
\hline ja & $1(3,4 \%)$ & $4(3,1 \%)$ & $1(25 \%)$ & $17(5,6 \%)$ & $23(5 \%)$ & 0,2438 \\
\hline nein & $28(96,6 \%)$ & $125(96,9 \%)$ & $3(75 \%)$ & $284(94,4 \%)$ & $440(95 \%)$ & \\
\hline Total & $29(6 \%)$ & $129(27,9 \%)$ & $4(0,9 \%)$ & $301(65 \%)$ & $463(100 \%)$ & \\
\hline
\end{tabular}

\subsubsection{Ergebnisse der Fragebogenevaluation}

Ein sekundäres Zielkriterium der Studie war die Untersuchung, ob ein Zusammenhang zwischen der im Fragebogen zur präoperativen Blutungsanamnese erreichten Punktzahl und dem Auftreten relevanter perioperativer Blutungskomplikationen vorlag. Die im Fragebogen erreichten Punktwerte reichten bei Patienten mit Blutungskomplikationen von 0 bis 9 Punkten, mit einem Median von 2 Punkten, einem unteren Quartil von 0 und einem oberen von 4 Punkten. Die Werte der Patienten ohne solche Blutungskomplikationen streuten zwischen 0 und 18 Punkten, mit einem mittleren Wert von 2 Punkten sowie einem ersten Quartil von 0 und einem dritten von 4 Punkten.

Es fand sich keine signifikante Korrelation zwischen der Höhe des Fragebogen-Punktwerts und der Wahrscheinlichkeit des Auftretens perioperativer Blutungskomplikationen (p-Wert $=0,39)$. Eine grafische Darstellung der Fragebogen-Punktwerte im Zusammenhang mit relevanten Blutungskomplikationen im Gesamtkollektiv ist in Abbildung 13 dargestellt. Insgesamt fand sich für keines der zwölf im Fragebogen abgefragten Kriterien ein statistisch signifikanter Zusammenhang mit der Häufigkeit des Auftretens von Blutungskomplikationen. 


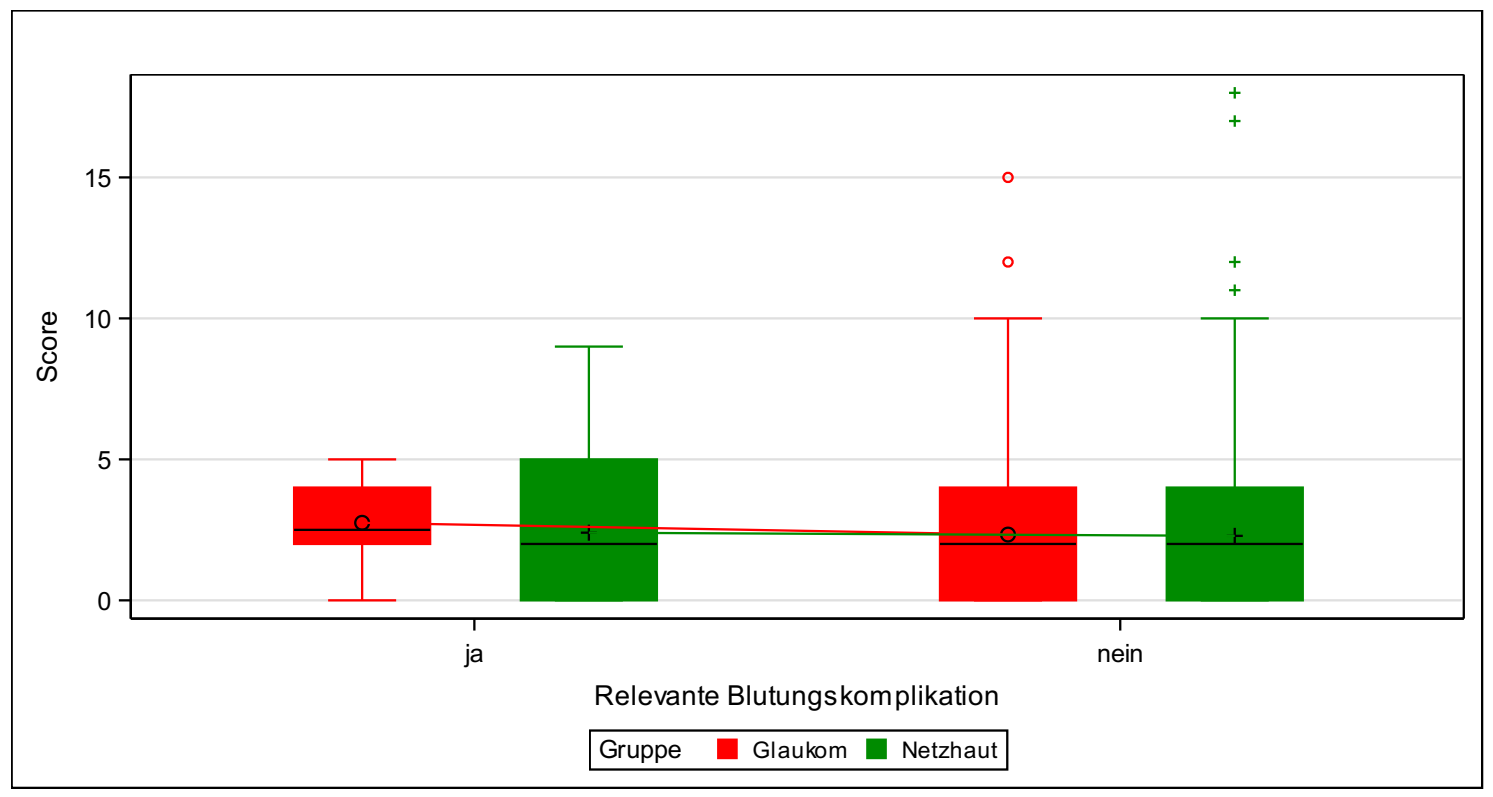

Abbildung 13: Blutungskomplikationen und Fragebogen-Score Vergleich der erreichten Punktwerte der Patienten mit und ohne relevante Blutungskomplikationen, aufgeteilt in Patienten mit Netzhaut- (grün) und Glaukomoperationen (rot)

\subsubsection{Ergebnisse der Blutdruckmessung}

Weder aus den vier intraoperativ erhobenen Blutdruckwerten (Anfangs- und Endwert sowie minimaler und maximaler Blutdruck), noch aus den postoperativen Werten konnten in den beiden Patientenkollektiven statistisch signifikante Unterschiede zwischen den Patienten mit und jenen ohne relevante Blutungskomplikationen festgestellt werden. Dies gilt sowohl für die systolischen als auch für die diastolischen Blutdruckwerte (alle $\mathrm{p}$-Werte $>0,05$ ). Dies ist auch in der nachfolgenden Abbildung 14 sichtbar, in der kein relevanter Unterschied zwischen den Blutdruckwerten bei Patienten mit und ohne relevante Blutungskomplikation gezeigt werden konnte. 


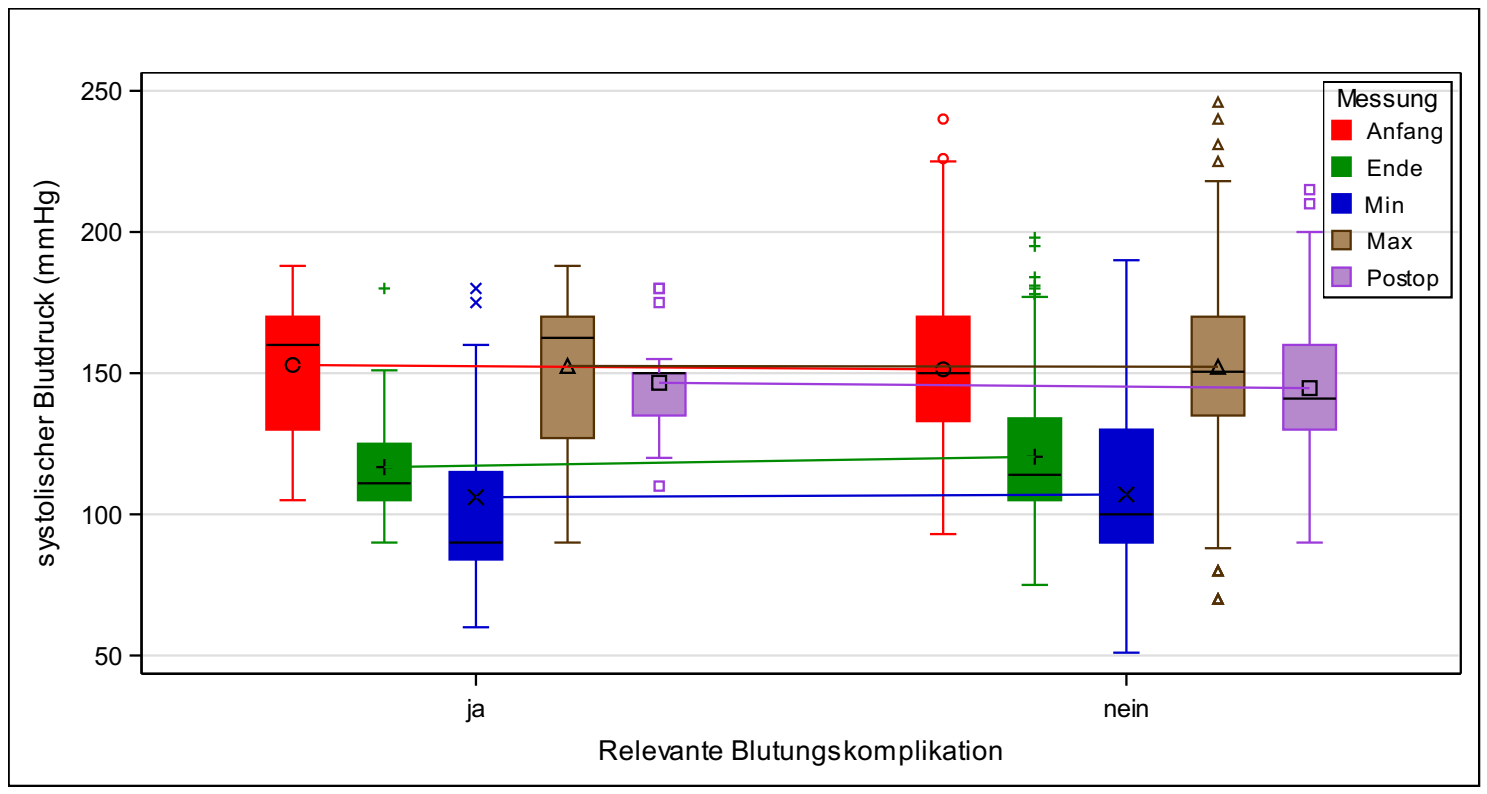

Abbildung 14: Blutungskomplikationen und systolischer Blutdruck bei Glaukom- und

Netzhautoperationen Vergleich mehrerer perioperativ erhobener systolischer Blutdruckwerte bei Patienten mit und ohne relevante Blutungskomplikationen

\subsubsection{Einfluss gerinnungsmodifizierender Medikamente}

Insgesamt nahm ein etwa gleich großer Teil der Glaukom- und Netzhautpatienten Medikamente ein, die die Gerinnung beeinflussen (

Tabelle 15).

Tabelle 15: Patienten mit gerinnungsmodifizierender Dauermedikation

\begin{tabular}{|l|l|l|l|}
\hline \multicolumn{1}{|c|}{ Gruppe } & \multicolumn{1}{c|}{ ja } & \multicolumn{2}{c|}{ nein } \\
\hline Glaukom & $32(36 \%)$ & $57(64 \%)$ & $89(19,2 \%)$ \\
\hline Netzhaut & $134(35,8 \%)$ & $240(64,2 \%)$ & $374(80,8 \%)$ \\
\hline Total & $166(35,9 \%)$ & $297(64,1 \%)$ & $463(100 \%)$ \\
\hline
\end{tabular}

Die Einnahme von gerinnungsmodifizierenden Medikamenten zeigte keinen statistisch signifikanten Zusammenhang mit relevanten Blutungskomplikationen ( $\mathrm{p}$-Wert $=0,22)$, wie in Tabelle 16 zu sehen. 
Um die Auswirkung der jeweiligen Medikamentengruppen (TFH, VKA, NOAK, NEM) zu sehen, wurde die Auswertung um eine einzelne Analyse für jede Gruppe ergänzt. ${ }^{8}$ Auch hier konnte bei keiner der Substanzklassen eine signifikante Korrelation zwischen deren Einnahme und einer erhöhten Rate von Blutungskomplikationen beobachtet werden.

Tabelle 16: Gerinnungsmodifizierende Medikamente und relevante Blutungskomplikationen bei Glaukom- und Netzhautoperationen

\begin{tabular}{|c|c|c|c|c|c|c|c|}
\hline \multirow[b]{2}{*}{$\begin{array}{c}\text { Gerinnungsmodifiziere } \\
\text { nde Medikamente }\end{array}$} & \multicolumn{2}{|c|}{$\begin{array}{c}\text { Relevante } \\
\text { Blutungskomplikation }\end{array}$} & & \multicolumn{3}{|c|}{ Chi square test } & \multirow{2}{*}{$\begin{array}{c}\text { Fisher's } \\
\text { exact } \\
\text { test } \\
\text { p-value }\end{array}$} \\
\hline & $\mathrm{ja}$ & nein & Total & DF & $\begin{array}{l}\text { Test- } \\
\text { statistic }\end{array}$ & p-value & \\
\hline ja & $11(47,8 \%)$ & $155(35,2 \%)$ & $166(35,9 \%)$ & 1 & 1,5085 & 0,2194 & 0,2652 \\
\hline nein & $12(52,2 \%)$ & $285(64,8 \%)$ & $297(64,1 \%)$ & . & . & . & . \\
\hline Total & $23(5 \%)$ & $440(95 \%)$ & $463(100 \%)$ & . & . & . & . \\
\hline \multicolumn{8}{|l|}{ TFH } \\
\hline ja & $8(7 \%)$ & $107(93 \%)$ & $115(24,8 \%)$ & 1 & 1,2821 & 0,2575 & 0,3201 \\
\hline nein & $15(4,3 \%)$ & $333(95,7 \%)$ & $348(75,2 \%)$ & . & . & . & . \\
\hline Total & $23(5 \%)$ & $440(95 \%)$ & $463(100 \%)$ & . & . & . & . \\
\hline \multicolumn{8}{|l|}{ VKA } \\
\hline ja & $2(12,5 \%)$ & $14(87,5 \%)$ & $16(3,5 \%)$ & 1 & 1,9918 & 0,1582 & 0,1857 \\
\hline nein & $21(4,7 \%)$ & $426(95,3 \%)$ & $447(96,5 \%)$ & . & . & . & . \\
\hline Total & $23(5 \%)$ & $440(95 \%)$ & $463(100 \%)$ & . & . & . & . \\
\hline \multicolumn{8}{|l|}{ NOAK } \\
\hline ja & $1(3,4 \%)$ & $28(96,6 \%)$ & $29(6,3 \%)$ & 1 & 0,1513 & 0,6973 & 1,0000 \\
\hline nein & $22(5,1 \%)$ & $412(94,9 \%)$ & $434(93,7 \%)$ & . & . & . & . \\
\hline Total & $23(5 \%)$ & $440(95 \%)$ & $463(100 \%)$ & . & . & . & . \\
\hline \multicolumn{8}{|l|}{ NEM } \\
\hline ja & $0(0 \%)$ & $9(100 \%)$ & $9(1,9 \%)$ & 1 & 0,4798 & 0,4885 & 1,0000 \\
\hline
\end{tabular}

8 Außerdem gab es noch einen Patienten, der Heparin einnahm, bei dem jedoch keine Blutungskomplikation auftrat, weshalb auf die Aufnahme in die Tabelle als einzelne Substanzgruppe verzichtet wurde. In die Gesamtauswertung wurde dieser jedoch aufgenommen. 


\begin{tabular}{|c|c|c|c|c|c|c|c|}
\hline \multirow[b]{2}{*}{$\begin{array}{c}\text { Gerinnungsmodifiziere } \\
\text { nde Medikamente }\end{array}$} & \multicolumn{2}{|c|}{$\begin{array}{c}\text { Relevante } \\
\text { Blutungskomplikation }\end{array}$} & & \multicolumn{3}{|c|}{ Chi square test } & \multirow{2}{*}{$\begin{array}{c}\text { Fisher's } \\
\text { exact } \\
\text { test } \\
\text { p-value }\end{array}$} \\
\hline & ja & nein & Total & DF & $\begin{array}{c}\text { Test- } \\
\text { statistic }\end{array}$ & p-value & \\
\hline nein & $23(5,1 \%)$ & $431(94,9 \%)$ & $454(98,1 \%)$ & . & . & . & \\
\hline Total & $23(5 \%)$ & $440(95 \%)$ & $463(100 \%)$ & . & . & . & \\
\hline
\end{tabular}

Zwischen der Dauer der Operation und einem erhöhten Auftreten von Blutungskomplikationen konnte eine Korrelation festgestellt werden. Die Dauer der Operationen bei Patienten mit relevanten Blutungskomplikation überstieg dabei die Dauer der Eingriffe bei Patienten ohne solche Komplikationen (siehe Abbildung 15). Dabei lag der p-Wert im T-Test für das Gesamtkollektiv bei 0,001 und die p-Werte bei den beiden Kohorten knapp über beziehungsweise knapp unter der Signifikanzgrenze von 5\% (Glaukomoperationen: 0,044, Netzhautoperationen: 0,053).

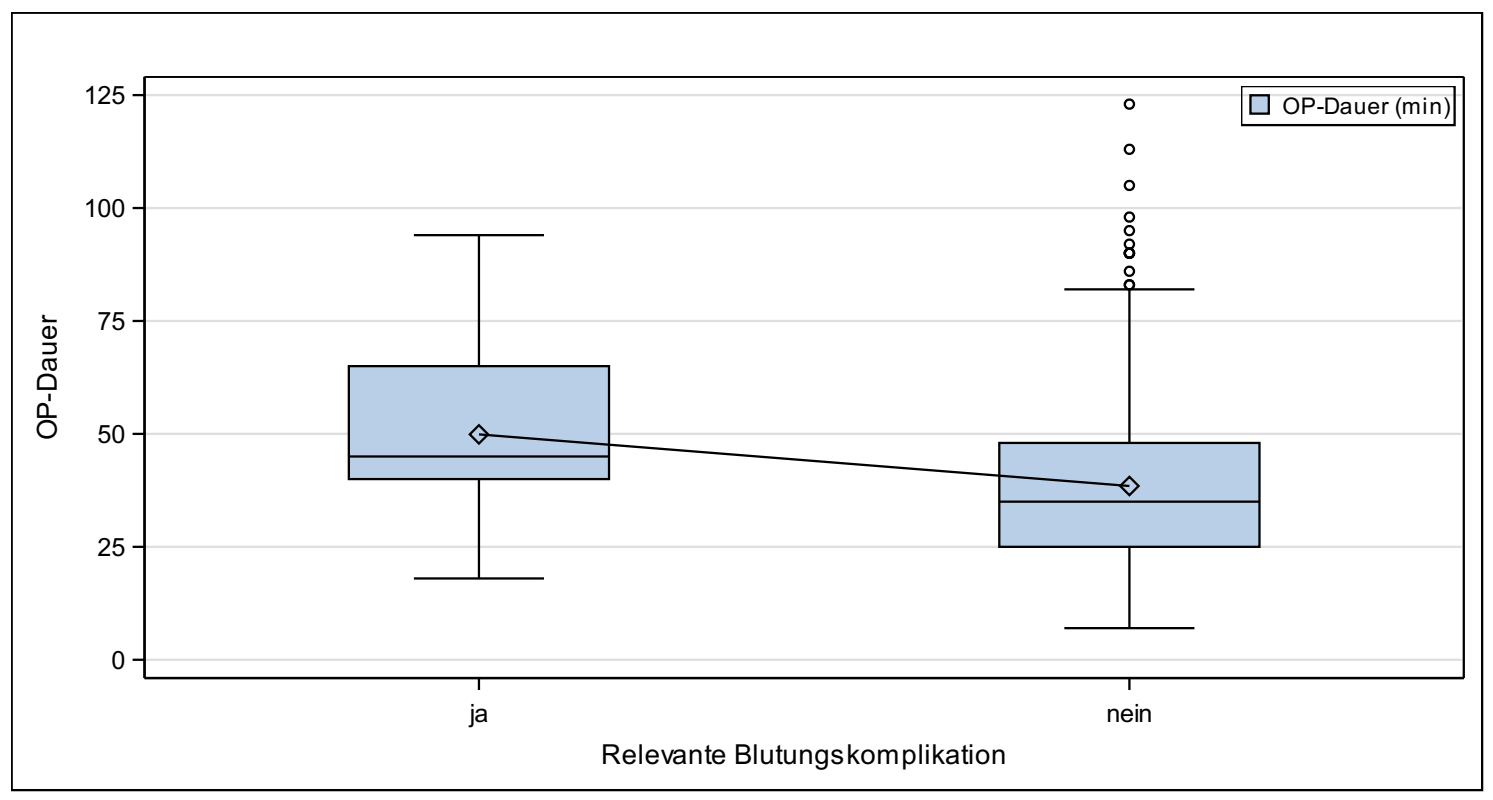

Abbildung 15: Blutungskomplikationen und OP-Dauer bei Netzhaut- und Glaukomoperationen (ja: $\mathrm{n}=23$, nein: $\mathrm{n}=440$ )

\subsubsection{Mögliche Risikofaktoren bei Netzhautoperationen}

Für das Kollektiv der Netzhautpatienten, war, im Unterschied zum Kollektiv der Glaukompatienten, eine signifikante Korrelation des Auftretens relevanter Blutungskomplikationen mit dem Alter der Patienten zu sehen ( $\mathrm{p}$-Wert $=0,01)$. Die 
Patienten mit einer relevanten Blutungskomplikation waren im Mittel 59,5 Jahre alt (STD 13,6), während die Patienten ohne Blutungskomplikation 67,9 Jahre alt waren (STD 12,8) (siehe Abbildung 16). Es konnte für Blutungskomplikationen bei Netzhautoperationen kein signifikanter Zusammenhang mit dem Geschlecht gefunden werden.

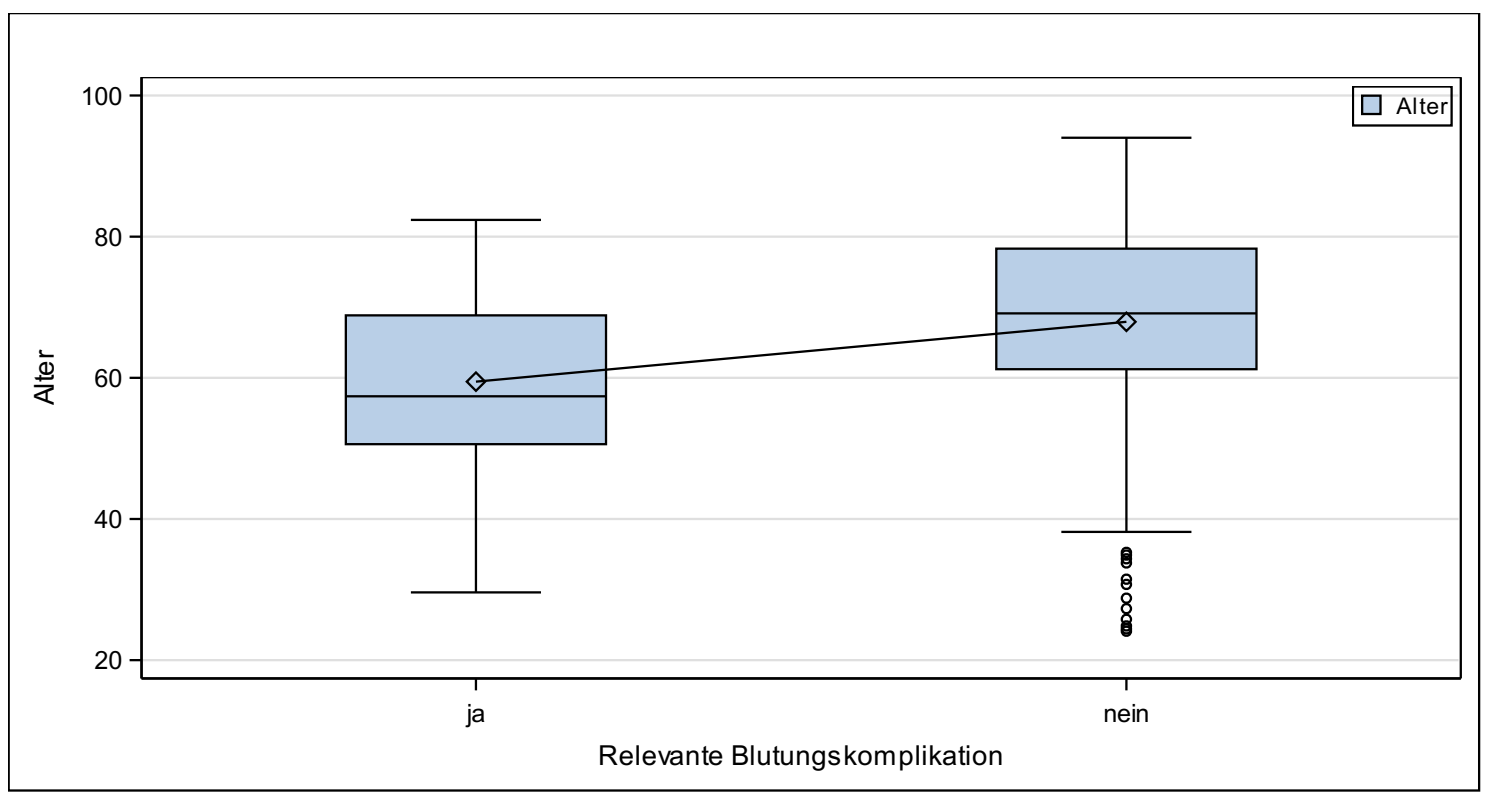

Abbildung 16: Blutungskomplikationen und Alter bei Netzhautoperationen ( $\mathrm{ja}: \mathrm{n}=23$, nein: $\mathrm{n}=440$ )

\subsubsection{Einfluss kardiovaskulärer Begleiterkrankungen}

Begleiterkrankungen, die besonders häufig bei Blutungskomplikationen auftraten, waren arterielle Hypertonie, die bei neun $(60 \%)$ der Patienten mit Blutungskomplikationen diagnostiziert war, und Diabetes mellitus, bei sieben (46,7\%) Patienten. Der prozentuale Anteil der Patienten mit arterieller Hypertonie an jenen mit einer relevanten Blutungskomplikation (60\%) entspricht dabei etwa dem Anteil der arteriellen Hypertoniker am Gesamtkollektiv der Netzhautpatienten (61,0\%). Im Gegensatz dazu übersteigt der Anteil der an Diabetes mellitus Erkrankten in der Gruppe mit Blutungskomplikationen (46,7\%) deren Anteil an allen Studienpatienten (20,1\%) erheblich. Gleiches gilt für Patienten mit einer Karotis-Stenose $(20 \%$ bei den Patienten mit Blutungskomplikationen, 2,94\% insgesamt). Sowohl für Diabetes mellitus $(\mathrm{p}$-Wert $=0,016)$ als auch für die Karotis-Stenosen $(\mathrm{p}$-Wert $=0,007)$ konnte eine statistisch signifikante Korrelation in Fishers exaktem Test gezeigt werden. Darüber hinaus traten auch bei Patienten, die keine kardiovaskulären Begleiterkrankungen hatten, relevante Blutungskomplikationen auf. Der Anteil dieser Patienten ohne Begleiterkrankungen entspricht prozentual in der Gruppe der Patienten mit Blutungen (26,7\%) etwa dem am betrachteten Kollektiv (29,9\%). Weitere relevante 
Begleiterkrankungen und deren relative Häufigkeit bei den Patienten mit relevanten Blutungskomplikationen sind in Tabelle 17 aufgeführt.

Tabelle 17: Kardiovaskuläre Begleiterkrankungen bei Patienten mit Netzhautoperationen

\begin{tabular}{|c|c|c|c|c|}
\hline \multirow[b]{2}{*}{ Relevante Begleiterkrankung } & \multicolumn{2}{|c|}{$\begin{array}{c}\text { Relevante } \\
\text { Blutungskomplikation }\end{array}$} & \multirow[b]{2}{*}{ Total } & \multirow[b]{2}{*}{$\begin{array}{c}\text { Fisher's } \\
\text { exakter Test, } \\
\text { p-Wert }\end{array}$} \\
\hline & $\mathrm{Ja}$ & Nein & & \\
\hline arterielle Hypertonie & $9(60 \%)$ & $218(60,7 \%)$ & $227(60,7 \%)$ & 1,0000 \\
\hline Keine & $4(26,7 \%)$ & $108(30,1 \%)$ & $112(29,9 \%)$ & 1,0000 \\
\hline Diabetes mellitus & $7(46,7 \%)$ & $68(18,9 \%)$ & $75(20,1 \%)$ & 0,0163 \\
\hline Koronare Herzkrankheit & $3(20 \%)$ & $38(10,6 \%)$ & $41(11 \%)$ & 0,2196 \\
\hline Tiefe Beinvenenthrombose & $1(6,7 \%)$ & $34(9,5 \%)$ & $35(9,4 \%)$ & 1,0000 \\
\hline Stent & $1(6,7 \%)$ & $26(7,2 \%)$ & $27(7,2 \%)$ & 1,0000 \\
\hline Vorhofflimmern & $0(0 \%)$ & $22(6,1 \%)$ & $22(5,9 \%)$ & 1,0000 \\
\hline Schlaganfall & $2(13,3 \%)$ & $18(5 \%)$ & $20(5,3 \%)$ & 0,1879 \\
\hline Herzinfarkt & $0(0 \%)$ & $17(4,7 \%)$ & $17(4,5 \%)$ & 1,0000 \\
\hline Nierenerkrankung & $1(6,7 \%)$ & $14(3,9 \%)$ & $15(4 \%)$ & 0,4653 \\
\hline periphere arterielle Verschlusskrankheit & $0(0 \%)$ & $15(4,2 \%)$ & $15(4 \%)$ & 1,0000 \\
\hline Karotis-Stenose & $3(20 \%)$ & $8(2,2 \%)$ & $11(2,9 \%)$ & 0,0071 \\
\hline Bypass & $0(0 \%)$ & $7(1,9 \%)$ & $7(1,9 \%)$ & 1,0000 \\
\hline Herzschrittmacher & $0(0 \%)$ & $6(1,7 \%)$ & $6(1,6 \%)$ & 1,0000 \\
\hline Lungenarterienembolie & $0(0 \%)$ & $5(1,4 \%)$ & $5(1,3 \%)$ & 1,0000 \\
\hline Immobilisation um OP-Zeitpunkt & $1(6,7 \%)$ & $3(0,8 \%)$ & $4(1,1 \%)$ & 0,1516 \\
\hline Herzklappenersatz & $0(0 \%)$ & $3(0,8 \%)$ & $3(0,8 \%)$ & 1,0000 \\
\hline Lebererkrankung & $1(6,7 \%)$ & $2(0,6 \%)$ & $3(0,8 \%)$ & 0,1159 \\
\hline Dialysepflichtig & $0(0 \%)$ & $2(0,6 \%)$ & $2(0,5 \%)$ & 1,0000 \\
\hline
\end{tabular}




\subsubsection{Einfluss der Operationsform}

Die Häufigkeit relevanter Blutungen wurden außerdem auf ihren Zusammenhang mit den durchgeführten Eingriffen untersucht. Bei Netzhautoperationen blutete es am häufigsten bei Patienten mit eindellenden Operationen bei einer Netzhautablösung. Insgesamt ist auch der Anteil der eindellenden Operationen unter den Patienten mit Blutungskomplikationen deutlich höher als unter allen Patienten mit einer Netzhautoperation. Es hat also von den Patienten mit relevanten Blutungskomplikationen ein relativ hoher Anteil eine eindellende Operation erhalten. Dies galt für Patienten mit einer alleinigen Plombenoperation, die an allen Netzhauteingriffen einen Anteil von 9,6\% (36 Patienten) hatten, während 26,7\% der Patienten (vier Personen) mit relevanten Blutungskomplikationen einen solchen Eingriff erhielten. Gleiches ist bei Patienten, die eine Kombination aus Pars-Plana-Vitrektomie und Eindellung erhielten, zu beobachten: 13,3\% der Patienten (zwei Personen) mit Blutungskomplikationen hatte einen solchen Eingriff, insgesamt aber nur ein Anteil von 7,0\% (26 Personen). Noch deutlicher ist ein solcher Unterschied bei Patienten mit einer Vitrektomie bei diabetischer Retinopathie zu beobachten. Während fünf $(33,3 \%)$ der Patienten mit relevanten Blutungskomplikationen einen solchen Eingriff bekamen, stellten ppV bei diabetischer Retinopathie insgesamt nur 5,0\% (19) aller Netzhauteingriffe. Bei den häufigsten Operationen, der ppV bei epiretinaler Gliose und bei Makulaforamen, kam es nicht zu relevanten Blutungskomplikationen. Die einzelnen Häufigkeiten aller erfolgten Eingriffe und deren prozentuale Verteilung auf Patienten mit und ohne relevante Blutungskomplikationen sind in Tabelle 18 dargestellt. 
Tabelle 18: Blutungskomplikationen bei verschiedenen Netzhautoperationen

\begin{tabular}{|c|c|c|c|}
\hline \multirow[b]{2}{*}{ Eingriff } & \multicolumn{2}{|c|}{$\begin{array}{c}\text { Relevante } \\
\text { Blutungskomplikation }\end{array}$} & \multirow[b]{2}{*}{ Total } \\
\hline & $\mathrm{Ja}$ & Nein & \\
\hline ppV bei epiretinaler Gliose & 0 & $73(20,33 \%)$ & $73(19,52 \%)$ \\
\hline ppV bei Makulaforamen & 0 & $62(17,27 \%)$ & $62(16,58 \%)$ \\
\hline ppV bei Netzhautablösung & $3(20,00 \%)$ & $56(15,60 \%)$ & $59(15,78 \%)$ \\
\hline ppV mit Öl-Entfernung & $3(20,00 \%)$ & $52(14,48 \%)$ & $55(14,71 \%)$ \\
\hline Plombenoperation (inklusive Cerclage) bei Netzhautablösung & $4(26,67 \%)$ & $32(8,91 \%)$ & $36(9,63 \%)$ \\
\hline Kombinationseingriff (Eindellung und ppV) & $2(13,33 \%)$ & $24(6,69 \%)$ & $26(6,95 \%)$ \\
\hline ppV bei Glaskörperblutung & $1(6,67 \%)$ & $23(6.41 \%)$ & $24(6,42 \%)$ \\
\hline ppV bei Subluxation der Linse/mit IOL-Einnähung & 0 & $24(6,69 \%)$ & $24(6,42 \%)$ \\
\hline ppV bei diabetischer Retinopathie & $5(33,33 \%)$ & $14(3,90 \%)$ & $19(5,08 \%)$ \\
\hline ppV bei submakulärer Blutung/Maculablutung & $1(6,67 \%)$ & $16(4,46 \%)$ & $17(4,55 \%)$ \\
\hline ppV bei Entzündung & 0 & $3(0,84 \%)$ & $3(0,80 \%)$ \\
\hline ppV bei Glaskörpertrübung & $1(6,67 \%)$ & $2(0,56 \%)$ & $3(0,80 \%)$ \\
\hline ppv bei Z.n. Gefäßverschluss & 0 & $2(0,56 \%)$ & $2(0,53 \%)$ \\
\hline
\end{tabular}

Zusätzlich zeigte sich eine klinisch relevante Korrelation zwischen der Blutungshäufigkeit und der Durchführung einer transskleralen Punktion bei Netzhautoperationen (

Tabelle 19) (aufgrund der geringen Fallzahlen keine Berechnung der Signifikanz).

Tabelle 19: Blutungskomplikationen und transsklerale Punktion bei eindellenden

Netzhautoperationen

\begin{tabular}{|c|c|c|c|}
\hline \multirow[b]{2}{*}{ Relevante Blutungskomplikation } & \multicolumn{2}{|c|}{ Transsklerale Punktion } & \multirow[b]{2}{*}{ Total } \\
\hline & ja & nein & \\
\hline ja & $5(71,4 \%)$ & $2(28,6 \%)$ & 7 \\
\hline nein & $16(8,6 \%)$ & $169(91,4 \%)$ & 185 \\
\hline Total & 21 & 171 & 192 \\
\hline
\end{tabular}




\subsubsection{Mögliche Risikofaktoren bei Glaukomoperationen}

Im Gegensatz zu den Netzhautpatienten konnte bei den Glaukomeingriffen keine signifikante Korrelation zwischen dem Patientenalter und der Blutungswahrscheinlichkeit nachgewiesen werden $(\mathrm{p}$-Wert $=0,6)$. Hingegen bestand ein Zusammenhang zwischen dem männlichen Geschlecht und der Blutungshäufigkeit (Tabelle 20).

Tabelle 20: Blutungskomplikationen und Geschlecht bei Glaukomoperationen

\begin{tabular}{|c|c|c|c|c|c|c|c|}
\hline \multirow[b]{2}{*}{$\begin{array}{c}\text { Relevante } \\
\text { Blutungskomplika } \\
\text { tion }\end{array}$} & \multicolumn{2}{|c|}{ Geschlecht } & & \multicolumn{3}{|c|}{ Chi square test } & \multirow{2}{*}{$\begin{array}{l}\text { Fisher's } \\
\text { exact test } \\
\text { p-value }\end{array}$} \\
\hline & weiblich & männlich & Total & DF & $\begin{array}{c}\text { Test- } \\
\text { statistic }\end{array}$ & p-value & \\
\hline ja & $1(12,5 \%)$ & $7(87,5 \%)$ & $8(9 \%)$ & 1 & 6,4335 & 0,0112 & 0,0204 \\
\hline nein & $48(59,3 \%)$ & $33(40,7 \%)$ & $81(91 \%)$ & . & . & . & . \\
\hline Total & $49(55,1 \%)$ & $40(44,9 \%)$ & $89(100 \%)$ & . & & . & \\
\hline
\end{tabular}

\subsubsection{Einfluss kardiovaskulärer Begleiterkrankungen}

Auch bei den Patienten mit Eingriffen im Rahmen einer Glaukomerkrankung wurden kardiovaskuläre Begleiterkrankungen erhoben. Bei Blutungskomplikationen im Rahmen von Glaukomoperationen war die arterielle Hypertonie die häufigste Begleiterkrankung und lag bei 50,0\% $(n=4)$ der Patienten mit Blutung vor. Insgesamt hatten 66,3\% $(n=59)$ der Patienten eine bereits diagnostizierte arterielle Hypertonie. Eine Tiefe Beinvenenthrombose war anamnestisch bei 37,5\% ( $\mathrm{n}=3)$ der Patienten mit Blutungskomplikationen aufgetreten, insgesamt bei $11,2 \%$ der Glaukompatienten. Außerdem waren unter den Patienten mit Blutungskomplikationen eine pAVK und das Vorliegen eines Stents häufiger vertreten. Für die TBVT und pAVK ist dieser Unterschied auch statistisch signifikant (p-Werte: 0,04 für TBVT und 0,039 für pAVK). Alle erfassten kardiovaskulären Begleiterkrankungen der Patienten mit Glaukomoperationen sind zur Übersicht in Tabelle 21 dargestellt. 
Tabelle 21: Blutungskomplikationen und kardiovaskuläre Begleiterkrankungen bei

\section{Glaukomoperationen}

\begin{tabular}{|c|c|c|c|c|}
\hline \multirow[b]{2}{*}{ Relevante Begleiterkrankung } & \multicolumn{2}{|c|}{$\begin{array}{l}\text { Relevante } \\
\text { Blutungskomplikation }\end{array}$} & \multirow[b]{2}{*}{ Total } & \multirow[b]{2}{*}{$\begin{array}{c}\text { Fisher's } \\
\text { exakter Test, } \\
\text { p-Wert }\end{array}$} \\
\hline & $\mathrm{Ja}$ & Nein & & \\
\hline arterielle Hypertonie & $4(50 \%)$ & $55(67,9 \%)$ & $59(66,3 \%)$ & 0,4347 \\
\hline Keine & $0(0 \%)$ & $20(24,7 \%)$ & $20(22,5 \%)$ & 0,1906 \\
\hline Diabetes mellitus & $2(25 \%)$ & $10(12,3 \%)$ & $12(13,5 \%)$ & 0,2935 \\
\hline Tiefe Beinvenenthrombose & $3(37,5 \%)$ & $7(8,6 \%)$ & $10(11,2 \%)$ & 0,0431 \\
\hline Koronare Herzkrankheit & $1(12,5 \%)$ & $8(9,9 \%)$ & $9(10,1 \%)$ & 0,5896 \\
\hline Schlaganfall & $1(12,5 \%)$ & $7(8,6 \%)$ & $8(9 \%)$ & 0,5446 \\
\hline Stent & $2(25 \%)$ & $6(7,4 \%)$ & $8(9 \%)$ & 0,1507 \\
\hline Herzinfarkt & $1(12,5 \%)$ & $6(7,4 \%)$ & $7(7,9 \%)$ & 0,4953 \\
\hline Vorhofflimmern & $1(12,5 \%)$ & $6(7,4 \%)$ & $7(7,9 \%)$ & 0,4953 \\
\hline Karotis-Stenose & $0(0 \%)$ & $4(4,9 \%)$ & $4(4,5 \%)$ & 1,0000 \\
\hline Lungenarterienembolie & $1(12,5 \%)$ & $3(3,7 \%)$ & $4(4,5 \%)$ & 0,3186 \\
\hline Periphere arterielle Verschlusskrankheit & $2(25 \%)$ & $2(2,5 \%)$ & $4(4,5 \%)$ & 0,0390 \\
\hline Nierenerkrankung & $1(12,5 \%)$ & $1(1,2 \%)$ & $2(2,2 \%)$ & 0,1726 \\
\hline Bypass & $0(0 \%)$ & $1(1,2 \%)$ & $1(1,1 \%)$ & 1,0000 \\
\hline Herzklappenersatz & $0(0 \%)$ & $1(1,2 \%)$ & $1(1,1 \%)$ & 1,0000 \\
\hline
\end{tabular}

\subsubsection{Einfluss der Operationsform}

Auch für Glaukomoperationen wurde die Verteilung der Blutungskomplikationen auf die verschiedenen Eingriffe untersucht. Die höchste Rate an relevanten Blutungskomplikationen trat bei der Trabekulektomie auf. Bei 50,0\% ( $\mathrm{n}=4)$ der Patienten mit Blutungskomplikationen war ein solcher Eingriff durchgeführt worden, während die Trabekulektomie nur einen Anteil von 33,7\% $(\mathrm{n}=30)$ an allen Glaukomeingriffen hatte. Bei Trabekulotomien mit dem Trabektom und bei Viskokanaloplastiken kam es ebenfalls zu Blutungskomplikationen. Auch bei Kanaloplastiken traten, gemessen an ihrem Gesamtanteil an allen Glaukomeingriffen, überdurchschnittlich häufig Blutungskomplikationen auf. Im Gegensatz dazu waren Trabektom-Operationen bei Patienten mit Blutungen (25,0\%) (n = 
2), verglichen mit der Häufigkeit dieses Eingriffs beim Gesamtkollektiv (42,7\%) ( $\mathrm{n}=38)$, unterrepräsentiert. Hierbei war jedoch auffällig, dass bei beiden Trabektom-Eingriffen, bei denen eine relevante Blutungskomplikation auftrat, dieser Eingriff in Kombination mit einer Kataraktoperation durchgeführt wurde. Die einzelnen Häufigkeiten der relevanten Blutungskomplikationen bei Glaukomoperationen sind in Tabelle 22 dargestellt.

Tabelle 22: Blutungskomplikationen bei verschiedenen Glaukomoperationen

\begin{tabular}{|l|l|l|l|}
\hline \multicolumn{1}{|c|}{ Eingriff } & \multicolumn{2}{c}{\begin{tabular}{c} 
Relevante \\
\multicolumn{1}{|c|}{ Blutungskomplikation }
\end{tabular}} \\
\hline Trabektom & \multicolumn{1}{|c|}{ Ja } & \multicolumn{1}{c|}{ Nein } & Total \\
\hline Trabekulektomie & $2(25,00 \%)$ & $36(44,44 \%)$ & $38(42,70 \%)$ \\
\hline Kanaloplastik & $4(50,00 \%)$ & $26(32,10 \%)$ & $30(33,71 \%)$ \\
\hline Kahook & $2(25,00 \%)$ & $7(8,64 \%)$ & $9(10,11 \%)$ \\
\hline Ventil-Implantation (Ahmed, Baerveldt) & 0 & $6(7,41 \%)$ & $6(6,74 \%)$ \\
\hline
\end{tabular}

\subsection{Patienten mit Blutungskomplikationen}

Die 23 Patienten mit relevanten perioperativen Blutungskomplikationen sollten nochmals betrachtet werden, um gemeinsame Parameter oder Auffälligkeiten herauszufinden. Aufgrund der geringen Anzahl an Patienten und der großen Anzahl an erhobenen Merkmalen ist eine statistische Analyse, bei der eine solche Kombination gezeigt werden könnte, nicht sinnvoll. Es konnten jedoch bei diesen Patienten keine Parameter gefunden werden, die auf eine typische Konstellation bei Patienten mit Blutungskomplikationen hinweisen könnten. Eine Übersicht aller Patienten mit relevanten perioperativen Blutungskomplikationen ist in Tabelle 23 und Tabelle 24 dargestellt. Zusammenfassend lässt sich sagen, dass sich trotz der Größe des untersuchten Kollektivs aus der vorliegenden Studie keine Hinweise auf ein bestimmtes Muster erkennen lassen, welches auf ein erhöhtes Blutungsrisiko bei den betrachteten intraokularen Eingriffen hinweist. 
Tabelle 23: Präoperative Merkmale der Patienten mit perioperativen Blutungskomplikationen

\begin{tabular}{|c|c|c|c|c|c|}
\hline $\begin{array}{l}\text { Pati } \\
\text { ent }\end{array}$ & Gruppe & $\begin{array}{l}\text { Alter bei } \\
\text { Eingriff } \\
\text { in Jahren }\end{array}$ & Geschlecht & $\begin{array}{l}\text { kardiovaskuläre } \\
\text { Begleiterkrankungen }\end{array}$ & Gerinnungshemmer \\
\hline 1 & Glaukom & 73 & männlich & $\begin{array}{l}\text { arterielle Hypertonie } \\
\text { KHK } \\
\text { Herzinfarkt } \\
\text { TBVT } \\
\text { Diabetes mellitus } \\
\text { Nierenfunktionseinschränkung }\end{array}$ & TFH \\
\hline 2 & Glaukom & 58 & männlich & $\begin{array}{l}\text { Schlaganfall } \\
\text { Diabetes mellitus }\end{array}$ & TFH \\
\hline 3 & Glaukom & 79 & männlich & $\begin{array}{l}\text { Lungenarterienembolie } \\
\text { Tiefe Beinvenenthrombose }\end{array}$ & nein \\
\hline 4 & Glaukom & 76 & weiblich & arterielle Hypertonie & nein \\
\hline 5 & Glaukom & 80 & männlich & arterielle Hypertonie & nein \\
\hline 6 & Glaukom & 74 & männlich & Vorhofflimmern & NOAK \\
\hline 7 & Glaukom & 77 & männlich & $\begin{array}{l}\mathrm{pAVK} \\
\text { Stent }\end{array}$ & TFH \\
\hline 8 & Glaukom & 64 & männlich & $\begin{array}{l}\text { arterielle Hypertonie } \\
\text { Tiefe Beinvenenthrombose } \\
\text { pAVK } \\
\text { Stent }\end{array}$ & TFH \\
\hline 9 & Netzhaut & 70 & weiblich & arterielle Hypertonie & nein \\
\hline 10 & Netzhaut & 82 & weiblich & $\begin{array}{l}\text { arterielle Hypertonie } \\
\text { Tiefe Beinvenenthrombose } \\
\text { Karotis-Stenose }\end{array}$ & TFH \\
\hline 11 & Netzhaut & 59 & männlich & $\begin{array}{l}\text { arterielle Hypertonie } \\
\text { KHK } \\
\text { Diabetes mellitus }\end{array}$ & VKA \\
\hline 12 & Netzhaut & 65 & männlich & $\begin{array}{l}\text { arterielle Hypertonie } \\
\text { Diabetes mellitus }\end{array}$ & VKA \\
\hline 13 & Netzhaut & 47 & weiblich & keine & nein \\
\hline 14 & Netzhaut & 68 & weiblich & keine & nein \\
\hline 15 & Netzhaut & 55 & männlich & arterielle Hypertonie & nein \\
\hline 16 & Netzhaut & 50 & weiblich & Diabetes mellitus & nein \\
\hline 17 & Netzhaut & 29 & männlich & $\begin{array}{l}\text { arterielle Hypertonie } \\
\text { Diabetes mellitus } \\
\text { Leberfunktionseinschränkung }\end{array}$ & nein \\
\hline 18 & Netzhaut & 45 & männlich & keine & nein \\
\hline 19 & Netzhaut & 54 & männlich & arterielle Hypertonie & TFH \\
\hline
\end{tabular}




\begin{tabular}{|c|c|c|c|c|c|}
\hline $\begin{array}{l}\text { Pati } \\
\text { ent }\end{array}$ & Gruppe & $\begin{array}{l}\text { Alter bei } \\
\text { Eingriff } \\
\text { in Jahren }\end{array}$ & Geschlecht & $\begin{array}{l}\text { kardiovaskuläre } \\
\text { Begleiterkrankungen }\end{array}$ & Gerinnungshemmer \\
\hline & & & & $\begin{array}{l}\text { Schlaganfall } \\
\text { Diabetes mellitus }\end{array}$ & \\
\hline 20 & Netzhaut & 57 & weiblich & $\begin{array}{l}\text { arterielle Hypertonie } \\
\text { KHK } \\
\text { Stent } \\
\text { Karotis-Stenose }\end{array}$ & TFH \\
\hline 21 & Netzhaut & 55 & männlich & $\begin{array}{l}\text { arterielle Hypertonie } \\
\text { KHK } \\
\text { Schlaganfall } \\
\text { Karotis-Stenose } \\
\text { Diabetes mellitus } \\
\text { Nierenfunktionseinschränkung } \\
\text { Immobilisation zum Zeitpunkt } \\
\text { der OP }\end{array}$ & TFH \\
\hline 22 & Netzhaut & 78 & männlich & keine & nein \\
\hline 23 & Netzhaut & 67 & männlich & Diabetes mellitus & nein \\
\hline
\end{tabular}

Tabelle 24: Intraoperative Merkmale von Patienten mit perioperativen Blutungskomplikationen

\begin{tabular}{|l|l|l|l|l|l|l|l|}
\hline Patient & Eingriff & $\begin{array}{l}\text { Anästhesie- } \\
\text { form }\end{array}$ & $\begin{array}{l}\text { OP- } \\
\text { Dauer }\end{array}$ & $\begin{array}{l}\text { Intraoperati } \\
\text { ve Blutung } \\
\text { (Grad, } \\
\text { Blutungsqu } \\
\text { elle })\end{array}$ & $\begin{array}{l}\text { Nachblu } \\
\text { tung }\end{array}$ & $\begin{array}{l}\text { Visusbe } \\
\text { drohung }\end{array}$ & $\begin{array}{l}\text { Re- } \\
\text { OP }\end{array}$ \\
\hline 1 & Kanaloplastik & Parabulbär & & 4, Sklera/ZK & nein & nein & nein \\
\hline 2 & Trabekulektomie & ITN & 40 & $\begin{array}{l}3, \mathrm{KW} \\
3, \text { Sklera/ZK }\end{array}$ & nein & nein & nein \\
\hline 3 & Trabekulektomie & ITN & 45 & $\begin{array}{l}3, \text { KW } \\
3, \text { Sklera/ZK }\end{array}$ & nein & nein & nein \\
\hline 4 & Trabekulektomie & ITN & 45 & 3, Sklera/ZK & ja & nein & nein \\
\hline 5 & Trabektom & Tropf & 94 & 5, Aderhaut & ja & ja & ja \\
\hline 6 & Trabektom & ITN & 65 & 3, Sklera/ZK & nein & nein & nein \\
\hline 7 & Kanaloplastik & ITN & 40 & 3, Sklera/ZK & nein & nein & nein \\
\hline 8 & Kanaloplastik & ITN & 18 & 3, Bindehaut & ja & nein & nein \\
\hline 9 & Plombenoperation & ITN & 48 & 3, Netzhaut & ja & nein & nein \\
\hline
\end{tabular}

\footnotetext{
${ }^{9}$ Bei einigen Eingriffen wurde die OP-Dauer nicht erfasst.
} 


\begin{tabular}{|c|c|c|c|c|c|c|c|}
\hline Patient & Eingriff & $\begin{array}{l}\text { Anästhesie- } \\
\text { form }\end{array}$ & $\begin{array}{l}\text { OP- } \\
\text { Dauer }\end{array}$ & $\begin{array}{l}\text { Intraoperati } \\
\text { ve Blutung } \\
\text { (Grad, } \\
\text { Blutungsqu } \\
\text { elle) }\end{array}$ & $\begin{array}{l}\text { Nachblu } \\
\text { tung }\end{array}$ & $\begin{array}{l}\text { Visusbe } \\
\text { drohung }\end{array}$ & $\begin{array}{l}\text { Re- } \\
\text { OP }\end{array}$ \\
\hline 10 & $\begin{array}{l}\text { Kombinationseing } \\
\text { riff (Eindellung } \\
\text { und } \mathrm{ppV} \text { ) }\end{array}$ & ITN & 45 & 3, Netzhaut & nein & nein & nein \\
\hline 11 & $\mathrm{ppV}$ & ITN & 78 & 3, Netzhaut & nein & nein & nein \\
\hline 12 & $\mathrm{ppV}$ & Retrobulbär & & 3, Netzhaut & nein & nein & nein \\
\hline 13 & Plombenoperation & ITN & 40 & 4, Aderhaut & nein & nein & nein \\
\hline 14 & $\mathrm{ppV}$ & Retrobulbär & & keine & nein & nein & ja \\
\hline 15 & $\mathrm{ppV}$ & ITN & 45 & 3, Aderhaut & nein & nein & nein \\
\hline 16 & Eindellung & ITN & 75 & 3 , Netzhaut & nein & nein & nein \\
\hline 17 & $\mathrm{ppV}$ & ITN & 51 & 3, Netzhaut & nein & nein & nein \\
\hline 18 & $\mathrm{ppV}$ & ITN & 40 & 3, Aderhaut & nein & nein & nein \\
\hline 19 & $\mathrm{ppV}$ & Retrobulbär & 45 & 3, Netzhaut & nein & nein & nein \\
\hline 20 & $\mathrm{ppV}$ & ITN & 69 & 3, Aderhaut & ja & nein & nein \\
\hline 21 & Eindellung & ITN & & 3, Netzhaut & ja & ja & nein \\
\hline 22 & $\mathrm{ppV}$ & Retrobulbär & 20 & keine & ja & ja & ja \\
\hline 23 & $\mathrm{ppV}$ & ITN & 45 & 3, Netzhaut & ja & nein & nein \\
\hline
\end{tabular}

Um eine Unabhängigkeit der Merkmale voneinander zu zeigen, wurde eine multivariate Analyse in Form einer logistischen Regression durchgeführt. Hierbei wurden die Variablen mit einem p-Wert $<0,2$ als tendenziell mögliche signifikante Einflussfaktoren im Modell belassen. In der Tabelle 25 sind diese Variablen dargestellt. 
Tabelle 25: Multivariate Analyse (logistische Regression)

\begin{tabular}{|l|r|r|r|}
\hline \multicolumn{4}{|c|}{ Type 3 Analysis of Effects } \\
Variable & DF & Whi-Square & Pr > ChiSq \\
\hline Netzhaut oder Glaukom & 1 & 5,1215 & 0,0236 \\
\hline Geschlecht & 1 & 2,2662 & 0,1322 \\
\hline Alter & 1 & 4,5549 & 0,0328 \\
\hline Seite des operierten Auges & 1 & 3,6198 & 0,0571 \\
\hline Ersteingriff oder Wiederholungseingriff & 1 & 3,5069 & 0,0611 \\
\hline Op-Dauer & 1 & 6,7449 & 0,0094 \\
\hline Kardiovaskuläre Begleiterkrankungen & 1 & 3,2740 & 0,0704 \\
\hline
\end{tabular}




\section{Diskussion}

Perioperative Blutungskomplikationen spielen in operativen Fächern, so auch in der Augenheilkunde, eine wichtige Rolle. Gerinnungsmodifizierende Medikamente stehen im Verdacht, das Risiko für perioperative Blutungskomplikationen zu erhöhen. In der vorliegenden prospektiven Arbeit sollte dieser Zusammenhang untersucht werden. Es konnte jedoch keine Korrelation zwischen der Einnahme gerinnungsmodulierender Medikamente und einem erhöhten Risiko für intraoperative Blutungskomplikationen gezeigt werden. Darüber hinaus wurden weitere Risikofaktoren für perioperative Blutungskomplikationen untersucht.

Insgesamt wurden die Daten von Patienten im Alter von 24 bis 94 Jahren erhoben. Der Altersdurchschnitt war mit 71,3 Jahren bei den Glaukompatienten und 67,6 Jahren bei den Netzhautpatienten etwa ähnlich. Obwohl die Krankheitsbilder also in jedem Erwachsenenalter vorkommen, handelt es sich bei Patienten mit einem solchen Eingriff also vor allem um Patienten im höheren Alter. Das entspricht auch Angaben aktueller wissenschaftlicher Veröffentlichungen, nach denen augenärztliche Konsultationen im höheren Lebensalter deutlich zunehmen (Wolfram und Pfeiffer 2012). Der Anteil von Männern und Frauen war in beiden Patientenkohorten ebenso ausgeglichen wie die Seite des operierten Auges, was für eine gute Verteilung erstellten Kollektivs spricht. Der Anteil der Patienten mit kardiovaskulären Begleiterkrankungen entsprach mit 70,1\% bei den Netzhautund 77,5\% bei den Glaukompatienten einer in der Literatur beschriebenen Prävalenz von kardiovaskulären Erkrankungen bei Patienten ab 65 Jahren von etwa 70\% (Braun und Strasser 2006). Gerinnungsmedikamente nahmen, wie in

Tabelle 15 dargestellt, in beiden Kollektiven mit ca. 36\% ähnlich viele Patienten ein, was ebenfalls für eine gleichmäßige Verteilung des zufällig ausgewählten Kollektivs spricht und einen Vergleichswert für die Prävalenz der Einnahme gerinnungshemmender Medikamente in der Ophthalmochirurgie geben könnte. Mit 500 Patienten ist das gewählte Kollektiv ungleich größer als sonst in der Augenheilkunde üblich. Darüber hinaus deuten die genannten Verteilungen insgesamt auf eine gute Repräsentativität des erstellten Kollektivs hin.

Die Darstellung der Ergebnisse diente vor allem der Vorstellung der Kollektive sowie der Aufzählung der Blutungskomplikationen und ihrer statistischen Zusammenhänge. Im Folgenden sollen die vorliegenden Ergebnisse nun zur Beantwortung der gewählten Forschungsfragen der vorliegenden Arbeit herangezogen und im Kontext aktueller Literatur betrachtet werden. 


\subsection{Anwendbarkeit des Fragebogens zur präoperativen Blutungsanamnese in der Ophthalmochirurgie}

Anhand dieser Arbeit sollte die Anwendbarkeit des Fragebogens zur präoperativen Blutungsanamnese in der Augenheilkunde überprüft werden (siehe auch Abbildung 5). Es konnte jedoch sowohl für das Gesamtkollektiv als auch jeweils für die Gruppen der Netzhaut- und Glaukompatienten kein Zusammenhang zwischen der Gesamtpunktzahl im Fragebogen und der Rate von Blutungskomplikationen gezeigt werden. Auch für die zwölf einzelnen Fragen bestand kein signifikanter Zusammenhang.

Der Fragebogen scheint somit nicht für die regelhafte Anwendung im Bereich der intraokularen Operationen der Ophthalmochirurgie geeignet zu sein. Die Fragen zur präoperativen Blutungsanamnese erfassen vor allem Risikofaktoren für Blutungen aus großlumigeren Gefäßen. Die Besonderheiten der Anatomie des Auges wie Blutungen aus sehr kleinen Gefäßen und vermutlich vordergründlich anderen Blutungsursachen, wie Sickerblutungen, werden nicht berücksichtigt. Zur weiteren Verringerung perioperativer Blutungskomplikationen bei intraokularen Eingriffen müssten also andere, wirkungsvollere Maßnahmen gefunden werden.

\subsection{System zur Einteilung der Blutungsstärke von intraoperativen Blutungen}

Im Rahmen dieser Studie wurde erstmals das hier vorgestellte Blutungsgrading (Tabelle 4 und Tabelle 5, Seite 22) zur untersucherunabhängigen Beurteilung intraoperativer Blutungen bei Netzhaut- und Glaukomeingriffen angewendet. Bei allen 463 Studienteilnehmern wurde das Grading unmittelbar postoperativ durch den Operateur vorgenommen. Die vorliegende Einteilung wurde im Konsens anhand der Auswirkungen erstellt, welche intraoperative Blutungen auf den Operationsverlauf hatten. Es sollte überprüft werden, ob eine solche Einteilung hilfreich und die getroffenen Abstufungen sinnvoll sind. Darüber hinaus sollte getestet werden, ob die Blutungen durch das erstellte Grading von nicht am Eingriff beteiligten Personen besser nachzuvollziehen und zu beurteilen sind. Des Weiteren sollte untersucht werden, ob die im Grading erfolgte Einstufung Hinweise auf den postoperativen Verlauf und klinische Auswirkungen für den Patienten geben kann.

Die Definition der Stufe 3 des Blutungsgradings musste bei Glaukomoperationen modifiziert werden. Stufe 3 bedeutete, es handelte sich um eine ,Blutung, für die die Diathermie eingesetzt werden muss, um danach die Operation störungsfrei fortzusetzen‘. Bei der Durchführung der Trabekulektomie wird zu einem bestimmten Zeitpunkt des Eingriffs 
immer die Diathermie eingesetzt, kleine intraoperative Blutungen dienen bei diesem Operationsverfahren dem Ophthalmochirurgen als Signal dafür, dass die richtigen Schichten erreicht sind. Daher war eine Unterscheidung zwischen einem regelhaften Einsatz der Diathermie und einem, der durch eine außergewöhnlich starke oder störende intraoperative Blutung nötig war, gefordert, damit nicht im Rahmen jeder Trabekulektomie die Stufe 3 im Blutungsgrading erreicht wurde. Im Verlauf der Studie wurde entschieden, dass dieser Punkt nur dann angekreuzt werden sollte, wenn es ohne den Einsatz der Diathermie zu einer subjektiv vom Operateur empfundenen wesentlichen Beeinträchtigung des Operationsverlaufes gekommen wäre. Der Fokus sollte hier also auf der Beurteilung durch den Arzt liegen, wie sehr er durch die Blutung im Operationsverlauf gestört wurde.

$\mathrm{Zu}$ Beginn der vorliegenden Arbeit wurden intraoperative Blutungen ab Stufe 3 im Blutungsgrading, die Notwendigkeit einer Re-Operation und eine dauerhafte Visusbedrohung als relevante Blutungskomplikationen definiert. Insgesamt war zu beobachten, dass es mit 21 Blutungen ab Stufe 3 deutlich mehr als relevant eingestufte intraoperative Blutungen gab, als Patienten, bei denen eine Re-Operation erforderlich war oder deren Visus dauerhaft bedroht war, wie

Tabelle 12 gezeigt.

Außerdem wurden die Re-Operationen, die bei den Patienten mit einer Netzhautoperation erforderlich waren, nicht durch im eigentlichen Sinne intraoperative Blutungen, sondern einmal durch eine postoperative Nachblutung und einmal durch eine Blutungskomplikation im Rahmen der Retrobulbäranästhesie verursacht. Es ist also festzuhalten, dass durch das Grading und die im Rahmen der Arbeit festgelegte Definition von relevanten Blutungskomplikationen zusätzliche Blutungen erfasst wurden, welche weder gemäß dem Artikel von Sucker et al. als relevante Blutungskomplikationen galten noch wesentliche klinische Auswirkungen für die Patienten hatten.

Zusammenfassend lässt sich sagen, dass das von den Augenchirurgen im Konsens erstellte Blutungsgrading eine neue klinisch anwendbare Definition bietet. Hierbei liegt der Fokus auf der Frage, wie sehr die Blutung die Operation stört und den Verlauf verzögert und weniger auf der Größe des Blutverlustes. Da diese bei intraokularen Eingriffen in der Augenheilkunde nur in seltenen Ausnahmefällen hämodynamisch relevant oder gar lebensbedrohlich für den Patienten ist, erscheint dieser Fokus aber durchaus sinnvoll. Dennoch wurden durch das Grading viele intraoperative Blutungen erfasst, die keine klinische Relevanz hatten. Also kann das erstellte Blutungsgrading im wissenschaftlichen Kontext für die Bewertung von intraoperativen Blutungen und die Erforschung von Risikofaktoren für intraoperative 
Blutungen in der Augenheilkunde sinnvoll sein. Für den klinischen Alltag scheint das Grading jedoch nicht von wesentlichem Nutzen.

\subsection{Risikofaktoren für ein erhöhtes Blutungsrisiko}

In dieser Arbeit sollten Merkmale von Patienten gefunden werden, die auf ein erhöhtes Blutungsrisiko in der Netzhaut- und Glaukomchirurgie hindeuten.

Obwohl es bei Glaukomoperationen relativ häufiger als bei Netzhautoperationen zu Blutungskomplikationen kam, erhöhen nach den Ergebnissen dieser Studie weder Glaukomnoch Netzhauteingriffe signifikant das Risiko für Blutungskomplikationen.

Auch die Art der Anästhesie scheint keinen Einfluss auf das Blutungsrisiko zu haben. Ergebnisse aus anderen Veröffentlichungen, nach denen die jeweilige Anästhesieform bei bestimmten Eingriffen eine Auswirkung auf das perioperative Blutungsrisiko hatten (Stang und Bartz-Schmidt 2019), konnten in dieser Arbeit nicht bestätigt werden.

Zwischen der Dauer der Operation und einem erhöhten Auftreten von perioperativen Blutungen konnte ein Zusammenhang gezeigt werden. Bei einer längeren Operationsdauer kam es sowohl bei den Netzhaut- als auch bei den Glaukompatienten signifikant häufiger zu Blutungskomplikationen. Vermutlich ist diese Korrelation nicht darauf zurückzuführen, dass infolge einer längeren Dauer mehr Blutungen auftreten. Wahrscheinlicher ist die alternative Erklärung, wonach sich die Dauer des Eingriffes beim Auftreten einer intraoperativen Blutung als Folge dieser Blutung verlängert, etwa durch das Stillen der Blutung oder durch andere infolge der Blutung nötige zusätzliche Operationsschritte. Dieses Ergebnis ist für die Suche nach Faktoren für ein erhöhtes Blutungsrisiko also vermutlich eher zu vernachlässigen.

Zwischen einer erhöhten Anzahl an perioperativen Blutungskomplikationen und intra- oder postoperativ höherem Blutdruck konnte kein signifikanter Zusammenhang gezeigt werden. Obwohl sich ein intraoperativ erhöhter Blutdruck in anderen Fachdisziplinen als Faktor für erhöhtes Blutungsrisiko gilt (Dünne et al. 2005), scheint bei den untersuchten Eingriffen der intraoperative Blutdruck für Blutungskomplikationen keine wesentliche Rolle zu spielen. Auch ein postoperativer Anstieg des Blutdrucks war nicht statistisch signifikant mit einer höheren Wahrscheinlichkeit perioperative Blutungskomplikationen assoziiert.

\subsubsection{Risikofaktoren bei Netzhautoperationen}

Es konnte bei Patienten mit einer Netzhautoperation ein Zusammenhang eines erhöhten Auftretens von Blutungskomplikationen mit einem niedrigeren Lebensalter gefunden werden. Dies könnte dadurch zu erklären sein, dass Blutungskomplikationen besonders 
häufig bei ppV im Rahmen einer diabetischen Retinopathie auftraten. Die Patienten mit dieser Erkrankung sind im Verhältnis zum betrachteten Kollektiv besonders jung. Des Weiteren traten Blutungskomplikationen besonders häufig im Rahmen eindellender Operationen bei Netzhautablösungen auf.

Einige der erhobenen kardiovaskulären Begleiterkrankungen kamen deutlich häufiger bei Patienten mit relevanten Blutungskomplikationen vor als im Gesamtkollektiv der Netzhautpatienten. Für die Erkrankung an Diabetes mellitus und Karotis-Stenosen fand sich eine signifikante Korrelation mit der Rate perioperativer Blutungskomplikationen. Die Korrelation eines erhöhten Anteils von Patienten mit Diabetes mellitus an Patienten mit perioperativen Blutungskomplikationen könnte darauf zurückzuführen sein, dass es bei Patienten mit einer Augenbeteiligung ihrer Diabeteserkrankung, einer diabetischen Retinopathie, besonders häufig zu perioperativen Blutungskomplikationen kam. Für den Grund des erhöhten Auftretens von Blutungskomplikationen bei Patienten mit einer Karotis-Stenose konnte keine begründete Hypothese aufgestellt werden. Zudem begrenzt die untersuchte Anzahl der Betroffenen mit elf Patienten mit einer Karotis-Stenose die Aussagekraft dieser Beobachtung.

Einige Eingriffe waren unter den Patienten mit Blutungskomplikationen überproportional häufig vertreten. Dazu gehört die ppV im Rahmen einer diabetischen Retinopathie. Diese Korrelation könnte darauf zurückgeführt werden, dass sich bei einer chirurgisch behandlungsbedürftigen diabetischen Retinopathie häufig Neovaskularisationen an der Netzhaut gebildet haben. Spontane Glaskörperblutungen kommen als Komplikation dieser Erkrankung vor (Gandorfer und Kampik 2000), darüber hinaus werden intraokulare Blutungen als häufigste Komplikation dieses Eingriffs genannt (Oldendoerp und Spitznas 1988). Dies könnten Gründe für die Überrepräsentation dieses Eingriffs bei den Patienten mit Blutungskomplikationen in der vorliegenden Studie sein.

Außerdem konnte ein höheres Auftreten von Blutungskomplikationen bei eindellenden Operationen, sowohl bei einfachen buckelchirurgischen Eingriffen als auch bei Kombinationen mit einer Vitrektomie, gezeigt werden. Buckelchirurgische Eingriffe könnten also ein erhöhtes Risiko für Blutungskomplikationen haben. Auch in wissenschaftlichen Veröffentlichungen gibt es Angaben, wonach die subretinale Blutung eine der häufigsten Komplikationen bei eindellenden Verfahren bei Netzhautoperationen darstellt (Abdullah et al. 2010).

Diese Eingriffe scheinen somit mit einem erhöhten Blutungsrisiko einherzugehen.

Die Durchführung einer transskleralen Punktion scheint ein zusätzlicher Risikofaktor für perioperative Blutungskomplikationen zu sein. Eine solche Punktion erfolgt nur im Rahmen 
eindellender Operationen. Insgesamt kam es bei 5 von 21 transskleralen Punktionen, also bei 23,8\%, zu einer relevanten perioperativen Blutungskomplikation. Die hierbei erhobenen Fallzahlen sind jedoch für definitive Aussagen recht klein, weshalb dieser Aspekt etwa als Grundlage für weitere Forschungsarbeiten dienen könnte.

Insgesamt lässt sich sagen, dass den Ergebnissen dieser Arbeit zufolge vor allem die Grunderkrankung des Auges (wie die diabetische Retinopathie), die Art der durchgeführten Operationen sowie Besonderheiten im Rahmen der Operation (wie eine transsklerale Punktion) die wesentlichen Risikofaktoren für perioperative Blutungskomplikationen bei Netzhauteingriffen darstellen.

\subsubsection{Risikofaktoren bei Glaukomoperationen}

Einige der erhobenen Merkmale korrelierten nur bei Patienten mit Glaukomoperationen mit der Wahrscheinlichkeit relevanter Blutungskomplikationen. Im Gegensatz zum Netzhautkollektiv scheint bei Glaukompatienten das Alter keinen Einfluss auf das perioperative Blutungsrisiko zu haben. Somit kann auch bei dieser Patientenkohorte ein höheres Lebensalter als Risikofaktor für perioperative Blutungskomplikationen gemäß den in dieser Studie erhobenen Daten vernachlässigt werden.

Insgesamt kam es bei männlichen Patienten signifikant häufiger zu Blutungskomplikationen als bei weiblichen. Da es für diesen Zusammenhang in der Literatur keine Hinweise gibt und auch im Rahmen dieser Forschungsarbeit kein plausibler Grund dafür gefunden wurde, sollte hierfür die Hypothese einer zufälligen statistischen Korrelation angenommen werden.

Begleiterkrankungen, die bei Glaukompatienten mit Blutungskomplikationen signifikant häufiger als im Gesamtkollektiv vorkamen, waren eine TBVT und eine pAVK. Darüber hinaus gab es auch einen deutlich größeren Anteil von Patienten mit Stent bei der Patientengruppe mit Blutungskomplikationen als im Gesamtkollektiv der Probanden mit einer Glaukomoperation. Hierfür konnte keine Begründung gefunden werden.

Eingriffe im Rahmen einer Glaukomerkrankung, bei denen es besonders häufig blutete, waren die Trabekulektomie und die Viskokanaloplastik. Vor dem Hintergrund, dass im Rahmen einer Trabekulektomie die verhältnismäßig größte Manipulation am Auge verursacht wird, erscheint es plausibel, dass es bei diesem Operationsverfahren am häufigsten zu relevanten Blutungskomplikationen kam. Die Wundfläche ist hierbei größer als etwa bei der Viskokanaloplastik und es kann dadurch zu größeren Blutungen, zum Beispiel aus dem Ansatz eines äußeren Augenmuskels kommen. Darüber hinaus wird der Augapfel eröffnet, wodurch es auch zu Blutungen aus der Basis der Iris und dem Ziliarkörper kommen kann, es ergeben sich also einfach mehr mögliche Quellen einer Blutung. 
Bei der Viskokanaloplastik könnte der hohe Anteil an Blutungskomplikationen daraus resultieren, dass die Blutung, mündlicher Mitteilung eines erfahrenen Glaukomchirurgen zufolge, den Operateur dort aufgrund der Lokalisation am meisten stört, da im Rahmen der Operation eine aufwändige Präparation des Schlemm-Kanals erfolgt, bei der Blutungen sehr hinderlich sind. Darum trage er dann - folgerichtig - vermutlich eher einen höheren Score im Blutungsgrading ein. Bei Trabekulotomien mit dem Trabektom kam es nur in solchen Fällen zu Blutungen, wenn diese in Kombination mit einer Katarakt-Operation durchgeführt wurden. Auch dies erscheint nach Einschätzung erfahrener Augenchirurgen plausibel, da durch die Kombination dieser beiden Eingriffe ein größeres Trauma verursacht wird als durch die Trabektom-Operation allein.

\subsection{Erkenntnisse zu Blutungskomplikationen, Schussfolgerungen für den Umgang mit gerinnungsmodulierenden Medikamenten in der Augenchirurgie}

Zusammenfassend lässt sich sagen, dass insgesamt nur wenige relevante Blutungskomplikationen auftraten. Im Vergleich zu aktuellen Studien, bei denen es bei 6,6\% der vitreoretinalen Eingriffe (Meillon et al. 2018) sowie bei $8 \%$ der durchgeführten Trabekulektomien (Jampel et al. 2005) zu relevanten Blutungsereignissen kam, ist die Rate an Blutungskomplikationen in unserer Studie ähnlich. Es traten dabei im Vergleich bei Glaukomoperationen mehr Blutungen (bei acht Patienten (9\%)) als bei Netzhautpatienten (15 (4\%)) auf (Tabelle 13).

Die Auswertung der Daten macht deutlich, dass es bislang offensichtlich keinen etablierten Standard für die präoperativen Anpassungen der Medikation gibt, der von allen vorbehandelnden Ärzten beachtet würde. Dies gilt einerseits für die Person, die für die Veränderung verantwortlich ist. Neben dem behandelnden Augenchirurgen griffen auch der Hausarzt, der niedergelassene Augenarzt oder der Patient selbst in die präoperative Umstellung der Gerinnungsmedikation ein. Dabei wurden sogar Medikamente wie NOAK oder auch TFH wie Clopidogrel im Vorfeld ohne das Eingreifen der Operateure durch einzelne Hausärzte mit Heparin gebridget, obwohl dies in der Fachliteratur wegen eines fehlenden Schutzes vor thromboembolischen Komplikationen ausdrücklich nicht empfohlen wird (Fleisher et al. 2007).

Insgesamt konnte - trotz des Umfangs der Studie - keine statistisch signifikante Risikoerhöhung unter der Einnahme von Medikamenten, die Einfluss auf die Blutgerinnung haben, beobachtet werden. Auch in der multivariaten Analyse zeigte sich keine statistisch 
signifikante Korrelation zwischen Medikamenteneinnahme und einer erhöhten Anzahl perioperativer Blutungskomplikationen (Tabelle 25). Auch der perioperative Umgang mit diesen Substanzen hatte nach den Ergebnissen dieser Arbeit keine erkennbare Auswirkung auf die Wahrscheinlichkeit von Blutungskomplikationen.

Zusammenfassend lässt sich sagen, dass in der Netzhaut- und Glaukomchirurgie, den Ergebnissen dieser Studie zufolge, das Risiko perioperativer Blutungskomplikationen unter der Einnahme von gerinnungsmodifizierenden Substanzen nicht signifikant erhöht zu sein scheint. Angesichts der mit einer perioperativen Umstellung der Gerinnungs-modulierenden Medikation verbundenen Risiken muss daher erwogen werden, diese gar nicht abzusetzen. Dies stimmt auch mit einigen Empfehlungen der aktuellen Literatur, etwa zum Umgang mit TFH in der Makulachirurgie (Louison et al. 2020) oder zu gerinnungshemmmenden Medikamenten in der vitreoretinalen Chirurgie (Meillon et al. 2018) überein. Darüber hinaus scheint es keinen Einfluss auf das perioperative Blutungsrisiko zu haben, wie präoperativ mit den Gerinnungsmedikamenten verfahren wird.

Abschließend kann daher festgestellt werden, dass der perioperative Umgang mit Gerinnungsmedikation im Hinblick auf das Risiko perioperativer Blutungskomplikationen bei intraokularen Eingriffen für den Opthalmochirurgen keine wesentliche Rolle spielt.

\subsection{Bewertung möglicher Fehlerquellen}

Alle Patienten, die in die Studie eingeschlossen wurden, wurden konsekutiv aufgenommen. Aufgrund der Größe der untersuchten Stichprobe und der oben dargestellten Verteilung der Merkmale der Patienten erachten wir das Patientenkollektiv als repräsentativ.

Bei der vorliegenden Studie handelt es sich lediglich um eine Beobachtungsstudie, bei der keine Intervention, wie etwa eine Veränderung der Medikation, stattfand. Der höchste Evidenzgrad hätte durch eine randomisierte kontrollierte Studie erreicht werden können, bei der zwei verschiedene, randomisierte Patientengruppen miteinander verglichen werden, von denen die eine Gruppe zum Beispiel die gerinnungshemmenden Medikamente beibehält, während die andere sie absetzt. Dies sollte in nachfolgenden Forschungsarbeiten möglichst berücksichtigt werden.

Eine weitere mögliche Fehlerquelle könnte das Ausfüllen des Gradings durch die jeweiligen Operateure sein. Hierbei kann nicht ausgeschlossen werden, dass verschiedene Operateure die Blutungen unterschiedlich bewerten. Somit könnte die Beurteilung möglicherweise durch den Operateur verzerrt worden sein. 
Zudem wurden in der vorliegenden Studie nur glaukom- und netzhautchirurgische Eingriffe untersucht. Die Übertragbarkeit der Ergebnisse auf andere opthalmochirurgische Eingriffe ist somit nicht gesichert.

\subsection{Ausblick}

Die in der vorliegenden Arbeit erhobenen Befunde können nur Hinweise auf mögliche Risikofaktoren für perioperative Blutungskomplikationen bei intraokularen Eingriffen geben, weil lediglich die Daten aller Patienten erhoben wurden und es keine Intervention mit Kontrollgruppe gab. Diese könnten und sollten nun in weiteren Forschungsarbeiten genauer untersucht werden. Dabei sollten gezielt die Blutungsrisiken bei Diabetischer Retinopathie sowie bei Plombenoperationen, insbesondere im Zusammenhang mit der Durchführung einer transskleralen Punktion, betrachtet werden. Darüber hinaus sollte das Blutungsrisiko bei Glaukomeingriffen am Kammerwinkel, wie der Trabekulektomie und der Viskokanaloplastik, nochmals näher betrachtet werden. Soweit nicht gegenläufige Forschungsergebnisse eine Verhaltensänderung nahelegen, könnte die Empfehlung abgeleitet werden, die gerinnungsmodulierende Ausgangsmedikation perioperativ unverändert fortzusetzen. Die Übertragbarkeit der gewonnenen Erkenntnisse auf weitere ophthalmochirurgische Eingriffe könnte Gegenstand zukünftiger Forschung sein. 


\section{$5 \quad$ Zusammenfassung}

Aufgrund der zunehmenden Häufigkeit gerinnungshemmender Medikamente in der Augenheilkunde und einer bisher unzureichenden Datenlage sollte in der vorliegenden prospektiven Arbeit ein umfassender Datensatz zu diesem Thema erstellt werden. Im Zentrum der Untersuchung stand (1) die Evaluation eines Fragebogens zur präoperativen Blutungsanamnese der Gruppe um die Berliner Gerinnungsexperten Koscielny und Sucker für die Anwendung in der Augenchirurgie; (2) die Überprüfung eines als Konsens der beteiligten Operateure der UMG erstellten Gradings zur standardisierten Bewertung und Dokumentation intraoperativer Blutungen; (3) die Identifikation von Risikofaktoren für das Auftreten perioperativer Blutungen bei intraokularen Eingriffen. Schließlich sollten (4) aus dem erstellten Datensatz Empfehlungen für den Umgang mit Gerinnungshemmern in der Augenheilkunde abgeleitet werden.

Im Rahmen einer prospektiven Studie wurden zwischen Februar und Dezember 2019 die Daten von 463 Patienten mit Netzhaut- und Glaukomeingriffen erhoben. Die Daten von 374 Netzhaut- und 89 Glaukompatienten wurden sowohl getrennt als auch zusammen ausgewertet. Als relevante Blutungskomplikationen wurden dabei solche perioperativen Blutungen definiert, die eine Re-Operation oder eine dauerhafte Visusbedrohung zur Folge hatten, sowie intraoperative Blutungen ab Stufe 3 im definierten Blutungsgrading.

Insgesamt traten bei 23 Patienten (5\%) relevante perioperative Blutungskomplikationen auf, davon bei 4\% (15) der Netzhaut- und 9\% (8) der Glaukompatienten.

Weder für die Narkoseart noch für den intra- und postoperativen Blutdruck oder die Einnahme von gerinnungshemmenden Medikamenten konnte im Gesamtkollektiv oder in einer der beiden untersuchten Kohorten ein signifikanter Zusammenhang mit dem perioperativen Blutungsrisiko nachgewiesen werden.

Hingegen korrelierte die Operationsdauer mit dem Blutungsereignis, wobei sich die beiden Parameter gegenseitig bedingen. Je mehr es blutet, desto länger ist die Operationsdauer zu erwarten. Bei Netzhautpatienten konnten für junge Patienten die Begleiterkrankungen Diabetes mellitus und Karotis-Stenose, das Vorliegen einer diabetischen Retinopathie sowie die Durchführung einer Plombenoperation mit einer transskleralen Punktion ein signifikanter Zusammenhang mit einem Blutungsereignis beobachtet werden. In der Gruppe der Glaukompatienten gingen männliches Geschlecht, die Begleiterkrankungen tiefe Beinvenenthrombose und periphere arterielle Verschlusskrankheit, das Vorliegen eines Stents, die Durchführung einer Trabekulektomie, einer Kanaloplastik, oder einer Trabektomoperation in Kombination mit einem Katarakteingriff mit einer signifikanten Erhöhung perioperativer Blutungskomplikationen einher. 
Es konnte kein statistisch signifikanter Zusammenhang zwischen dem im Fragebogen zur präoperativen Blutungsanamnese ermittelten Score und der Wahrscheinlichkeit perioperativer Blutungskomplikationen gefunden werden. Deshalb kann der in anderen chirurgischen Fächern etablierte Fragebogen zur Blutungsanamnese nicht in die Ophthalmochirurgie übertragen werden.

Das vorgeschlagene Blutungsgrading ermöglichte eine untersucherunabhängige standardisierte Beurteilung intraoperativer Blutungen, jedoch wurden einige intraoperative Blutungen dadurch als relevante Komplikation bewertet, die in der rückschauenden Bewertung keine klinischen Auswirkungen für den Patienten hatten. Trotzdem bietet das System erstmals die Möglichkeit, den Schweregrad einer intraoperativen Blutung zu bewerten.

Merkmale, die mit einem erhöhten Blutungsrisiko einher gehen, sind vor allem die Grunderkrankung des Auges und die Art des Eingriffs. Folgestudien könnten vor allem die diabetische Retinopathie (als Behandlungsanlass) und Plombeneingriffe (als Operationstechnik) als Risikofaktoren für Blutungskomplikationen bei Netzhautoperationen sowie kammerwinkelchirurgische Eingriffe bei Glaukomoperationen untersuchen.

Abschließend kann die Empfehlung ausgesprochen werden, gerinnungshemmende Medikamente bei intraokularen Eingriffen beizubehalten, um so das Risiko thromboembolischer Komplikationen zu verringern, ohne das Risiko einer perioperativen Blutung zu erhöhen. 


\section{$6 \quad$ Anhang}

Die für die Befragung der Pateinten verwendeten Arbeitsblätter sind, mit Ausnahme des bereits im Material- und Methodenteil vorgestellten Fragebogens zur Blutungsanamnese, im Folgenden aufgeführt (Abbildung A. 1 - A. 5).

Studie: Blutungsrisiko und perioperative Blutungskomplikationen bei ophthalmochirurgischen Eingriffen

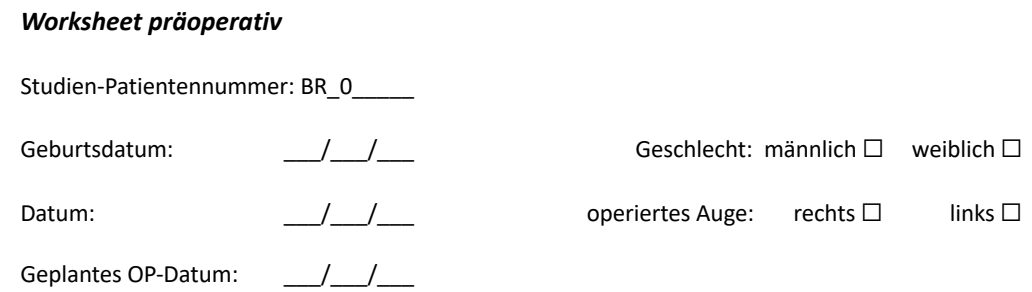

\begin{tabular}{|l|l|l|}
\hline Ein- und Ausschlusskriterien & ja & nein \\
\hline Operationsindikation Glaukom- oder Netzhautoperation & & \\
\hline Alter $>18$ & & \\
\hline Aufklärung und schriftliche Einwilligung des Patienten & & \\
\hline Score im Fragebogen zur Blutungsanamnese & & \\
\hline
\end{tabular}

Patientenaufnahme in die Studie, wenn alle grau hinterlegten Kästchen angekreuzt wurden.

2. Bitte kleben Sie einen Patientenaufkleber in die Wiedererkennungsliste im Ordner. Notieren Sie anschließend die Studien-Patientennummer oben auf dem Worksheet.

3. Händigen Sie dem Patienten die Patienteninformation aus und heften Sie die Einverständniserklärung des Patienten in dem Studienordner ab.

4. Fragebogen präoperativ

4.1. Diagnose/Eingriff:

Glaukomoperation $\square$

Trabekulektomie $\square \quad$ Ventil-Implantation (Ahmed, Baerveldt) $\square$

Xen $\square \quad$ iStent $\square \quad$ Trabektom $\square \quad$ Kahook $\square \quad$ Kanaloplastik $\square \quad$ +Katarakt $\square$

Netzhautoperation $\square$

ppV bei Makulaforamen $\square \quad$ ppV bei epiretinaler Gliose $\square \quad$ ppV bei diabetischer Retinopathie $\square$

ppV bei Netzhautablösung $\square \quad$ ppV bei submakuläre Blutung $\square \quad \operatorname{ppV~Glaskörpertrübung~} \square$

Plombenoperationen (inklusive Cerclage) bei Netzhautablösung $\square \quad$ ppV bei Entzündung $\square$

Kombinationseingriff (Eindellung und ppV) $\square \quad$ Ölentfernung $\square \quad$ +Katarakt $\square$

Besonderheiten:

Fragebogen Blutungskomplikationen bei Glaukom- oder Netzhauteingriffen 
4.2. kardiovaskuläre Begleiterkrankungen:

Art. Hypertonie $\square \quad$ KHK $\square \quad$ Herzinfarkt $\square \quad$ Stent $\square \quad$ Bypass $\square \quad$ Herzklappenersatz $\square$

Lungenarterienembolie $\square \quad$ TVBT $\square \quad$ pAVK $\square \quad$ Schlaganfall $\square \quad$ Vorhofflimmern $\square \quad$ Karotis-Stenose $\square$

Diabetes mellitus $\square \quad$ Lebererkrankung $\square \quad$ Nierenerkrankung $\square$. Dialysepflicht $\square \quad$ Immobilisation um OP $\square$

4.3. Absetzen oder Bridging von gerinnungshemmenden Medikamenten:

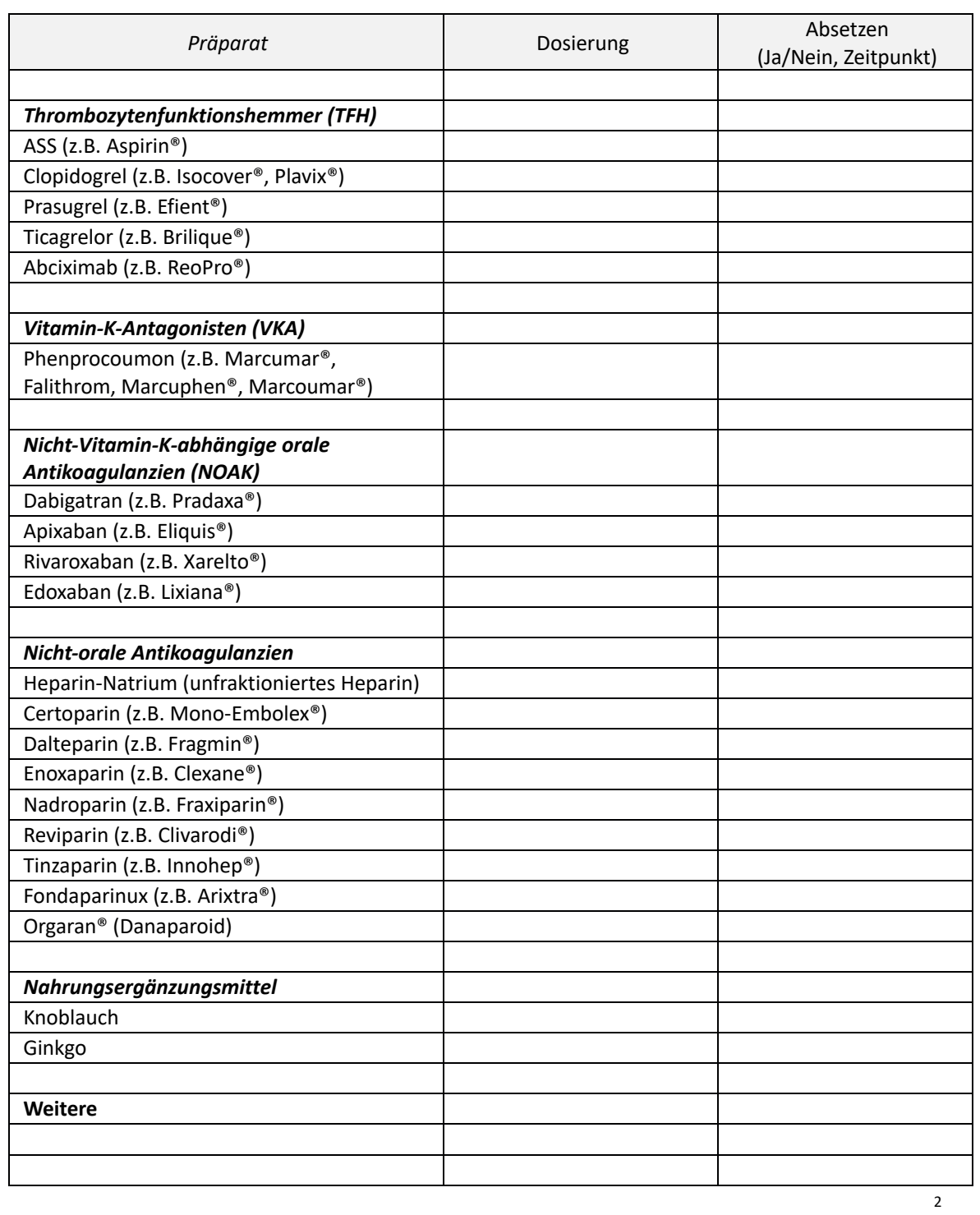

Fragebogen Blutungskomplikationen bei Glaukom- oder Netzhauteingriffen

Abbildung A. 2: Präoperatives Arbeitsblatt, Seite 2 
Studie: Blutungsrisiko und perioperative Blutungskomplikationen bei ophthalmochirurgischen Eingriffen

Worksheet intraoperativ

Studien-Patientennummer: BR_O

Ausfüllender Arzt:

Datum:____

1. Art der Narkose

Tropf $\square \quad$ Retrobulbär $\square \quad$ Parabulbär $\square \quad$ ITN $\square$

2. Ersteingriff $\square \quad$ Folgeeingriff $\square$

3. OP-Dauer: $\min$

4. intraoperativer RR [mmHg]:

$\begin{array}{ll}\text { Anfangs-RR: } & \text { End-RR: } \\ \text { Minimaler RR: }\end{array}$

5. Blutungs-Grading:

5.1 Netzhautoperationen

Bei eindellenden Operationen: transsklerale Punktion ja $\square$ nein $\square$

Grad 0: keine Blutung

Grad 1: Vitrektomie: spontan sistierende Blutung ohne Reduktion des Einblicks Buckelchirurgie: umschriebene/punktuelle subretinale Blutung in der Peripherie

Grad 2: Vitrektomie: Blutung, die nach vorübergehender Erhöhung des irrigationsdrucks zum Stillstand kommt, spontan sistierende Blutung mit Reduktion des Einblicks Buckelchirurgie: flächige subretinale Blutung auf die Peripherie begrenzt

Grad 3: Vitrektomie: Blutung, für die Endodiathermie eingesetzt werden muss Buckelchirurugie: flächige subretinale Blutung bis an die Gefäßbögen ohne Beteiligung des hinteren Pols

Grad 4: Vitrektomie: Blutung trotz Endodiathermie, die nur durch direkte Kompression gestillt werden kann (z.B. Staubsauger auf retinales Gefäß) Buckelchirurugie: flächige subretinale Blutung mit Beteiligung des hinteren Pols

Grad 5: Vitrektomie: nicht stillbare Blutung Buckelchirurgie: subretinale Massenblutung

\begin{tabular}{|l|l|l|l|}
\hline & Netzhaut & Aderhaut. & Sklera/ Ziliarkörper \\
\hline Grad 0 & & & \\
\hline Grad 1 & & & \\
\hline Grad 2 & & & \\
\hline Grad 3 & & & \\
\hline Grad 4 & & & \\
\hline Grad 5 & & & \\
\hline
\end{tabular}

Bitte bei Blutungen ab Stufe 3 eine Blutentnahme mit folgenden Werten anordnen: BB, Krea, GOT, GPT, INR, aPTT, Thrombozytenzahl, Thrombinzeit

Abbildung A. 3: Intraoperatives Arbeitsblatt, Seite 1 
5.2 Glaukomoperationen

Grad 0: keine Blutung

Grad 1: Spontan sistierende Blutungen

Grad 2: Blutungen, die den Operationsverlauf stören, die aber spontan bzw. ohne Einsatz von Diathermie sistieren (inkl. Applikation von Adrenalin, vorübergehende Erhöhung des Infusionsdrucks)

Grad 3: Blutung, für die Diathermie eingesetzt werden muss, danach Operation störungsfrei fortzusetzen

Grad 4: Blutung, die trotz Diathermie die Operation so stark behindern, dass sie nicht mehr weitergeführt werden kann

Grad 5: massive, nicht stillbare Blutung inklusive Aderhautblutung

\begin{tabular}{|l|l|l|l|l|}
\hline & Bindehaut & KW / Iris & Sklera /Ziliark. & Aderhaut \\
\hline Grad 0 & & & & \\
\hline Grad 1 & & & & \\
\hline Grad 2 & & & & \\
\hline Grad 3 & & & & \\
\hline Grad 4 & & & & \\
\hline Grad 5 & & & \\
\hline
\end{tabular}

Bitte bei Blutungen ab Stufe 3 eine Blutentnahme mit folgenden Werten anordnen: BB, Krea, GOT, GPT, INR, aPTT, Thrombozytenzahl, Thrombinzeit

Abbildung A. 4: Intraoperatives Arbeitsblatt, Seite 2 
Studie: Blutungsrisiko und perioperative Blutungskomplikationen bei ophthalmochirurgischen Eingriffen

\section{Worksheet postoperativ}

Studien-Patientennummer: BR_0

Ausfüllender Arzt:

Datum: ___ _ _ _

1. Im Fall von Nachblutungen:

\begin{tabular}{|l|l|l|l|l|}
\hline & Bindehaut & Netzhaut & Aderhaut & Sklera/ Ziliarkörper \\
\hline Blutungsquelle & & & & \\
\hline
\end{tabular}

2. postoperative Blutungskomplikationen: ja $\square \quad$ nein $\square \quad$ wenn ja, welche?:

3. dauerhaft visusbedrohende Blutungskomplikation? $\quad$ ja $\square \quad$ nein $\square$
wenn ja, welche?:

wenn ja, welche?

4. Komplikationen, die eine erneute OP nötig machen? ja $\square \quad$ nein $\square$

wenn ja, welche?:

5. Blutdruckwerte im Aufwachraum:

Minimaler RR: 1

Maximaler RR: 1

Abbildung A. 5: Postoperatives Worksheet 


\section{$7 \quad$ Literaturverzeichnis}

Abdullah AS, Jan S, Qureshi MS, Khan MT, Khan MD (2010): Complications of conventional scleral buckling occuring during and after treatment of rhegmatogenous retinal detachment. J Coll Physicians Surg Pak 20, 6

Agarwal PK, Mathew M, Virdi M (2010): Is there an effect of perioperative blood pressure on intraoperative complications during phacoemulsification surgery under local anaesthesia? Eye 24, 1186-1192

Albert FW, Eichler H, Haubelt H, Loreth R, Matzdorff A, Peetz D, Pindur G, Schinzel H, Seyfert U, Hellstern P (2009): Laboranalytischer Ausschluss einer hämorrhagischen Diathese vor elektiven Eingriffen? Ja! Hamostaseologie 29, 58-63

Bauersachs RM (2003): Therapie und Sekundärprophylaxe der venösen Thromboembolie mit Vitamin-K-Antagonisten. Internist 44, 1491-1499

Benzimra JD, Johnston RL, Jaycock P, Galloway PH, Lambert G, Chung AKK, Eke T, Sparrow JM (2009): The cataract national dataset electronic multicentre audit of 55567 operations: antiplatelet and anticoagulant medications. Eye $\underline{23}, 10-16$

Bonhomme F, Hafezi F, Boehlen F, Habre W (2013): Management of antithrombotic therapies in patients scheduled for eye surgery. Eur J Anaesthesiol $\underline{30}, 449-454$

Bopp S (2012): Subretinale Blutungen. Ophthalmologe 109, 635-643

Bopp S (2019): Eindellende Operationstechniken. Klin Monatsbl Augenheilkd 236, 603-618

Braun SD, Strasser RH (2006): Herz-Kreislauf-Erkrankungen. Gynäkologe 39, 354-361

Brown JS, Mahmoud TH (2011): Anticoagulation and clinically significant postoperative vitreous hemorrhage in diabetic vitrectomy. Retina $\underline{31}, 1983-1987$

Connolly SJ, Ezekowitz MD, Yusuf S, Eikelboom J, Oldgren J, Parekh A, Pogue J, Reilly PA, Themeles E, Varrone J et al. (2009): Dabigatran versus warfarin in patients with atrial fibrillation. New Eng J Med 361, 1139-1151

Dietlein TS, Hermann MM, Jordan JF (2009): Medikamentöse und chirugische Therapie des Glaukoms. Dtsch Ärztebl Int 106, 597-605

Douketis JD, Spyropoulos AC, Spencer FA, Mayr M, Jaffer AK, Eckman MH, Dunn AS, Kunz R (2012): Perioperative management of antithrombotic therapy: Antithrombotic therapy and prevention of thrombosis, 9th ed: American college of chest physicians evidence-based clinical practice guidelines. Chest 141, e326S-e350S

Dünne AA, Schüttler J, Sesterhenn A, Dalchow C, Folz B, Werner JA (2005): Intra- und frühe postoperative Blutungskomplikationen bei endonasaler Nasennebenhöhlenchirurgie - eine Analyse an 773 Patienten. HNO-Informationen (Kongressabstracts) 보, 498

Eberl W, Wendt I, Schroeder HG (2005): Präoperatives Screening auf Gerinnungsstörungen vor Adenotomie und Tonsillektomie. Klin Padiatr 217, 20-24

Eckardt C (2005): Transconjuntival sutureless 23-gauge vitrectomy. Retina 25, 208-211 
Eika C, Havig Ø, Godal HC (1978): The value of preoperative haemostatic screening. Scand J Haematol 21, 349-354

Eisele R, Melzer N, Bramlage P (2014): Perioperatives Gerinnungsmanagement bei oraler Antikoagulation. Chirurg 모, 513-519

Faude F, Meier P, Wiedemann P (2002): Eindellende Operationen bei rhegmatogener Amotio retinae. Ophthalmologe 99, 308-323

Feltgen N, Hoerauf H, Noske W, Hager A, Koscielny J (2016): Thrombozytenaggregationshemmer und Antikoagulantien bei augenchirurgischen Eingriffen. Ophthalmologe 113, 1010-1022

Feltgen N, Mele B, Dietlein T, Erb C, Eckstein A, Hager A, Heiligenhaus A, Helbig H, Hoerauf H, Hoffmann E et al. (2018): Umgang mit gerinnungshemmenden Substanzen in der Ophthalmochirurgie - eine Umfrage unter Ophthalmochirurgen in Deutschland. Ophthalmologe $115,585-591$

Fleisher LA, Beckman JA, Brown KA, Calkins H, Chaikof E, Fleischmann KE, Freeman WK, Froehlich JB, Kasper EK, Kersten JR et al. (2007): ACC/AHA 2007 guidelines on perioperative cardiovascular evaluation and care for noncardiac surgery. J Am Coll Cardiol $\underline{50}$, $1707-1732$

Gandorfer A, Kampik A (2000): Pars-plana-Vitrektomie bei diabetischer Retinopathie. Ophthalmologe 97, 325-330

Ghadimi K, Levy JH, Welsby IJ (2016): Perioperative management of the bleeding patient. Br J Anaesth 117, iii18-iii30

Giebl A, Gürtler K (2014): Neue orale Antikoagulanzien in der perioperativen Medizin. Anaesthesist 63, 347-364

Grehn F: Bindehaut. In: Grehn F (Hrsg.): Augenheilkunde (Springer-Lehrbuch). Springer, Heidelberg 2012, 87-108

Grehn F: Glaskörper, Vitrektomie. In: Grehn F (Hrsg.): Augenheilkunde. Springer, Heidelberg 2019, 357-374

Heimann H, Bartz-Schmidt KU, Bornfeld N, Weiss C, Hilgers RD, Foerster MH (2007): Scleral buckling versus primary vitrectomy in rhegmatogenous retinal detachment: a prospective randomized multicenter clinical study. Ophthalmology 114, 2142-2154

Hein L: Antikoagulantien und Thrombozytenaggregationshemmer. In: Schwabe U, Paffrath D (Hrsg.): Arzneiverordnungs-Report 2009: Aktuelle Daten, Kosten, Trends und Kommentare. Springer, Heidelberg 2009, 381-395

Heuser D, Decker K: Anästhesie bei Eingriffen am Auge. In: Rügheimer E (Hrsg.): Anästhesie für Operationen im Kopfbereich (Klinische Anästhesiologie und Intensivtherapie). Springer, Heidelberg 1988, 154-171

Hille K, Rossi M, Hille A, Ruprecht KW (2005): Die „Ahmed glaucoma valve“. Ophthalmologe $\underline{102}, 170-174$ 
Hoffmeister HM, Bode C, Darius H, Huber K, Rybak K, Silber S (2010): Unterbrechung antithrombotischer Behandlung (Bridging) bei kardialen Erkrankungen: Positionspapier. Kardiologe 4, 365-374

Jampel HD, Musch DC, Gillespie BW, Lichter PR, Wright MM, Guire KE (2005): Perioperative complications of trabeculectomy in the collaborative initial glaucoma treatment study (CIGTS). Am Journal Ophthalmol 140, 16-22

Jordan JF (2012): Minimal-invasive Kammerwinkelchirurgie. Ophthalmologe 109, 713-724

Jordan JF, Neuburger M, Reinhard T (2010): Minimal-invasive Kammerwinkelchirurgie. Ophthalmologe 107, 855-860

Koscielny J, Ziemer S, Radtke H, Schmutzler M, Pruss A, Sinha P, Salama A, Kiesewetter H, Latza R (2004): A practical concept for preoperative identification of patients with impaired primary hemostasis. Clin Appl Thromb Hemost 10, 195-204

Latza R, Koscielny J, Pruß A, Kiesewetter H (1999): Klinischer Einsatz von Desmopressin und Antifibrinolytika. Anästh Intensivmed 7/8, 548-553

Law SK, Song BJ, Yu F, Kurbanyan K, Yang TA, Caprioli J (2008): Hemorrhagic complications from glaucoma surgery in patients on anticoagulation therapy or antiplatelet therapy. Am J Ophthalmol 145, 736-746.e1

Lepper W, Kelm M (2007): Perioperative Therapie mit Thrombozytenaggregationshemmern. Anaesthesist 56, 592-598

Lock JF, Wagner J, Luber V, Dietz UA, Lichthardt S, Matthes N, Krajinovic K, Germer C -T, Knop S, Wiegering A (2018): Perioperativer Umgang mit Antikoagulation. Chirurg 모, 95-102 Louison S, Gabrielle PH, Soudry A, Meillon C, Blanc J, Béal G, Arsène S, Mer YL, Berrod JP, Kodjikian L et al. (2020): Perioperative risk of bleeding with antithrombotic agents in macular surgery: a national, prospective, multicentre study. Acta Ophthalmol 98, e991-997

Mason JO, Gupta SR, Compton CJ, Frederick PA, Neimkin MG, Hill ML, Heersink MJ, Vail RS, White MF, Feist RM et al. (2011): Comparison of hemorrhagic complications of warfarin and clopidogrel bisulfate in 25-gauge vitrectomy versus a control group. Ophthalmology $\underline{118}, 543$ 547

Meillon C, Gabrielle PH, Luu M, Aho-Glele LS, Bron AM, Creuzot-Garcher C, for the CFSR research net (2018): Antiplatelet and anticoagulant agents in vitreoretinal surgery: A prospective multicenter study involving 804 patients. Graefes Arch Clin Exp Ophthalmol 256, $461-467$

Merki R, Schwarb H, Tsakiris DA (2016): Direkte orale Antikoagulantien: Monitoring wann und wie? Ther Umsch $\underline{73}, 613-617$

Oh J, Smiddy WE, Kim SS (2011): Antiplatelet and anticoagulation therapy in vitreoretinal surgery. Am J Ophthalmol 151, 934-939 
Oldendoerp J, Spitznas M (1988): Der Einfluß von Rubeosis iridis und aktiven

Neovaskularisationen am Fundus auf die Ergebnisse der Glaskörperchirurgie bei der proliferativen diabetischen Retinopathie. Klin Monbl Augenheilkd 192, 650-659

Pfanner G, Koscielny J, Pernerstorfer T, Gütl M, Perger P, Fries D, Hofmann N, Innerhofer P,

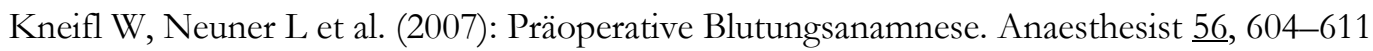

Piekarski F, Wunderer F, Raimann FJ, Neef V, Peuckert M, Brenner T, Grottke O, Grünewald M, Gürtler K, Renner J et al. (2020): Erfassung von intraoperativen Blutverlusten. Ergebnisse einer multizentrischen Erhebung und Überblick aktueller Methoden zur Quantifizierung von Blutverlusten. Anästh Intensivmed 61, 110-116.

Plange N: Glaukom. In: Walter P, Plange N (Hrsg.): Basiswissen Augenheilkunde (SpringerLehrbuch). Springer, Heidelberg 2017, 215-232

Reinthal EK, Rohrbach JM, Grisanti S (2010): Glaukom-Drainagechirurgie. Klin Monbl Augenheilkd 227, 49-55

Rex S (2001): Anästhesie in der Augenheilkunde. Anaesthesist 50, 798-815

Rohrschneider K, Greim S (2004): Erblindungsursachen in Baden von 1980 bis 1999. Klin Monbl Augenheilkd 221, 116-121

Scherer RU, Giebler RM (2004): Perioperative Gerinnungsstörungen. Anästhesiol Intensivmed Notfallmed Schmerzther $\underline{39}$, 415-443

Schlitt A, Jámbor C, Spannagl M, Gogarten W, Schilling T, Zwissler B (2013): The perioperative management of treatment with anticoagulants and platelet aggregation inhibitors. Dtsch Arztebl Int 110, 525-532

Schwenk W (2019): SOP Präoperative Untersuchung des Blutungsrisikos. Allgemein- und Viszeralchirurgie up2date $\underline{13}, 97-100$

Seicean A, Schiltz NK, Seicean S, Alan N, Neuhauser D, Weil RJ (2012): Use and utility of preoperative hemostatic screening and patient history in adult neurosurgical patients: Clinical article. J Neurosurg 116, 1097-1105

Sié P, Samama CM, Godier A, Rosencher N, Steib A, Llau JV, Van der Linden P, Pernod G, Lecompte T, Gouin-Thibault I et al. (2011): Surgery and invasive procedures in patients on long-term treatment with direct oral anticoagulants: Thrombin or factor-Xa inhibitors.

Recommendations of the Working Group on perioperative haemostasis and the French Study Group on thrombosis and haemostasis. Arch Cardiovasc Dis 104, 669-676

Sporbeck B, Bechara FG, Häfner HM, Koenen W, Kolk A, Koscielny J, Meissner M, Pokrywka A, Schirmer S, Strömer K et al. (2015): S3 guidelines for the management of anticoagulation in cutaneous surgery. J Dtsch Dermatol Ges $\underline{13}$, 346-356

Stang MM, Bartz-Schmidt KU (2019): Der Einfluss der Narkoseart auf die Häufigkeit revisionsbedürftiger postoperativer Nachblutungen nach Entfernung der Silikonölendotamponade. Klin Monbl Augenheilkd 236, 1439-1444 
Sucker C, Hager A, Koscielny J, Feltgen N (2019): Orale Antikoagulation: Aktuelle Übersicht und perioperatives Management in der Ophthalmochirurgie. Ophthalmologe 116, 144-151

Taruttis T, Chankiewitz E, Hammer T (2018): Vergleich von Trabekulektomie und Kanaloplastik. Ophthalmologe 115, 137-144

van Oterendorp C, Bahlmann D (2019): Kahook Dual Blade. Ophthalmologe 116, 580-584

Vogel Kahmann I, Ruppen W, Lurati Buse G, Tsakiris DA, Bruggisser M (2011):

Langzeitmedikation und perioperatives Management. Internist $\underline{52}$, 89-98

Völcker D, Junker B, Hansen LL (2012): Spontane Makulablutung. Ophthalmologe 109, 702-706

Walton W, Von Hagen S, Grigorian R, Zarbin M (2002): Management of traumatic hyphema. Surv Ophthalmol 47, 297-334

Wecker T, Jordan JF (2015): Minimalinvasive Kammerwinkelchirurgie mit dem Trabektom. Klin Monbl Augenheilkd 232, 303-309

Weindler J, Weindler M, Ruprecht KW (2004): Lokalanästhesie in der Ophthalmochirurgie. Ophthalmologe 101, 847-864

Wolfram C, Pfeiffer N: Weißbuch zur Situation der ophthalmologischen Versorgung in Deutschland. Deutsche Opthalmologische Gesellschaft, München 2012 


\section{Danksagung}

An dieser Stelle möchte ich Allen meinen großen Dank aussprechen, die mich bei der Anfertigung meiner Dissertation begleitet und unterstützt haben.

Besonders danke ich dabei zunächst Herrn Prof. Dr. Nicolas Feltgen für die hervorragende Betreuung und großartige Unterstützung bei der Umsetzung der gesamten Arbeit. Er hat mich sowohl außerordentlich gefördert als auch gefordert.

Prof. Dorothee Mielke danke ich für die sehr gute Betreuung dieser Arbeit und ihre konstruktiven Vorschläge bei gemeinsamen Treffen.

Darüber hinaus möchte ich den Spezialisten für Hämatologie, PD Dr. Jürgen Koscielny und PD Dr. Christian Sucker aus Berlin, meinen Dank aussprechen, die mir im Rahmen dieser Arbeit bei Fragen jederzeit weitergeholfen haben.

Mein Dank gilt auch dem gesamten weiteren Team der Augenklinik der Universitätsmedizin Göttingen, welches mich bei der Durchführung der Studie unterstützt haben, insbesondere PD Dr. Christian van Oterendorp. 